\title{
SPHERICAL FUNCTIONS ON CARTAN MOTION GROUPS
}

\author{
CARY RADER
}

\begin{abstract}
This paper gives a reasonably complete treatment of harmonic analysis on Cartan motion groups. Included is an explicit parameterization of irreducible spherical functions of general $K$-type, and of the nonunitary dual (and its topology). Also included is the explicit Plancherel measure, the Paley Wiener theorem, and an asymptotic expansion of general matrix entries. (These are generalized Bessel functions.) However the main result is Theorem 19 , a technical result which measures the size of the centralizer of $K$ in the universal enveloping algebra of the corresponding reductive group.
\end{abstract}

Introduction. This paper gives a reasonably complete treatment of the harmonic analysis of Cartan motion groups. We recall the definition. Let $G$ be a connected semisimple Lie group and let $K \subseteq G$ be a maximal compact subgroup. Let $\mathfrak{g}=\mathfrak{k}+\mathfrak{s}$ be the orthogonal (Cartan) decomposition of the Lie algebra of $G$. Then the Cartan motion group associated to $G$ is the semidirect product $H=K \times \mathfrak{s}$ using the adjoint representation of $K$ on $\mathfrak{s}$.

In $\S 4$ of this paper we obtain an explicit parameterization of the nonunitary dual of the Cartan motion group $H$ (Lemma 22), and of the Fell topology on it (Theorem 24). (Lemma 25 is an interesting general result on Fell topologies.) Mackey's theory of unitary induction is ideally suited to Cartan motion groups, making the computation of the unitary dual easy. It turns out that the nonunitary dual is just about what you might expect if Mackey's machine worked for nonunitary representations (but of course it does not). In Theorem 28 we find an explicit expression for the Plancherel measure of $H$, including the normalizing constant.

In $\S 5$, Theorem 35, and its corollary, is the Paley-Wiener theorem for these groups. Lemma 30 and Corollary 33 give a result on the asymptotic growth of the matrix entries of these representations. They use a variation on the method of stationary phase, with complex parameters. (The matrix entries are essentially generalized Bessel functions.) I wish to thank the referee for suggesting this greatly improved version of the result.

Actually most of this paper is concerned with parameterizing these matrix entries, or spherical functions. In the first section, we gather the notation and definitions and some abstract nonsense used in the rest of the paper. These should be more or less familiar to anyone who knows semisimple theory, so no proofs are included.

$\S \S 2$ and 3 are devoted to the proof of Theorem 19, a sort of Chevalley restriction theorem. (This is related to Kostant's $J$ and $Q$ matrices.) Let $V, \tau$ be a unitary

Received by the editors August 10, 1987.

1980 Mathematics Subject Classification (1985 Revision). Primary 22E30, 22E45, 22E47, 43A90, 15A72; Secondary 33A40, 33A75, 14L30. 
double representation of $K$ and let $S(\mathfrak{s}, \tau)$ be the space of polynomial functions $f: \mathfrak{s} \rightarrow V$ satisfying

$$
f(\operatorname{Ad} k \cdot X)=\tau_{1}(k) f(X) \tau_{2}\left(k^{-1}\right) \quad(k \in K, X \in \mathfrak{s}) .
$$

Let $\mathfrak{a}_{\mathfrak{s}}$ be a Cartan subspace of $\mathfrak{s}$. Then the restriction $f \mapsto f \mid \mathfrak{a}_{\mathfrak{s}}$ takes $S(\mathfrak{s}, \tau)$ onto a space of polynomial functions on $\mathfrak{a}_{\mathfrak{s}}$. Theorem 19 gives a characterization of the image of this restriction map. Now a spherical function is an "eigenfunction" for an algebra of differential operators isomorphic to $S(\mathfrak{s}, \tau)$ for an appropriate $V, \tau$ (see Proposition 7). Thus the problem of parameterizing the spherical functions becomes the problem of parameterizing the irreducible representations of the algebra of "restrictions" of these differential operators. This is a solvable problem.

1. Notation and definitions. This section reviews the basic definitions and abstract nonsense propositions having to do with spherical functions and large compact subgroups. This material should be familiar to people conversant with semisimple theory. The basic references are [G, W1, pp. 304-359 and W2, pp. $1-43]$.

Let $G$ be a locally compact, separable topological group and let $K$ be a compact subgroup. Let $\hat{K}$ be the set of equivalence classes of irreducible unitary representations of $K$. For $\mu_{\mathfrak{o}}: K \rightarrow \mathscr{L}\left(E_{\mathfrak{o}}\right)$ in $\mathfrak{d} \in \hat{K}$, let

$$
\alpha_{\mathfrak{d}}(k)=d(\mathfrak{d}) \operatorname{tr} \mu_{\mathfrak{o}}\left(k^{-1}\right)
$$

be the normalized character (where $d(\mathfrak{d})$ is the degree of $\mathfrak{d}$ ). If $\pi: G \rightarrow \mathscr{L}(E)$ is a (continuous) representation on the complete, locally convex, topological vector space $E$, let $P(\mathfrak{d})=\pi\left(\alpha_{\mathfrak{o}}\right)$ and

$$
[\pi: \mathfrak{o}]=\operatorname{dim} \operatorname{Hom}_{K}\left(E_{\mathfrak{o}}, E\right)=d(\mathfrak{o})^{-1} \operatorname{rank} P(\mathfrak{d}),
$$

the multiplicity of $\mathfrak{d}$ in $\pi$.

As it turns out, all of the representations considered in this paper are admissible (Harish-Chandra), that is $[\pi: \mathfrak{d}] \leq M d(\mathfrak{d})$ for some constant $M>0$. In fact we will assume that $K$ is large in $G$, that is $M$ is independent of $\pi$ for $\pi$ TCI, since this holds for Cartan motion groups [W1, pp. 228, 305, 314].

DEFINITION. Let $\pi_{1}: G \rightarrow \mathscr{L}\left(E_{1}\right)$ and $\pi_{2}: G \rightarrow \mathscr{L}\left(E_{2}\right)$ be admissible representations. A Naimark intertwining operator $Q: E_{1} \rightarrow E_{2}$ is a linear operator with dense domain and closed graph such that if $m$ is a compactly supported measure on $G$, then the domain and range of $Q$ are stable under $\pi_{1}(m)$ and $\pi_{2}(m)$ respectively, and $Q \pi_{1}(m)=\pi_{2}(m) Q$. If in addition $Q$ is one-to-one and has dense range, we say that $\pi_{1}$ and $\pi_{2}$ are Naimark equivalent and write $\left[\pi_{1}\right]=\left[\pi_{2}\right]$. Let $[G]$ denote the set of Naimark equivalence classes of TCI representations.

PROPOSITION 1. The collection of admissible representations and Naimark intertwining operators forms an abelian category.

(This comes from the fact that a linear map, defined on the $K$-finite vectors and which intertwines the actions by $K$-finite functions, admits a unique closure [W1, p. 326].) Next we describe the algebras with which we will be concerned.

DEFINITION. For $f \in C_{c}(G)$ (or any other reasonable function space on $G$ ) define $f^{\circ}$ by

$$
f^{\circ}(x)=\int_{K} \alpha_{\mathfrak{d}} * f\left(k x k^{-1}\right) d k .
$$


Let $I_{c \mathrm{D}}(G)=\left\{f \in C_{c}(G) \mid f^{\circ}=f\right\}$, a convolution algebra with the subspace topology.

DEFINITION. If $G$ is a connected Lie group, let $\mathfrak{G}$ be its universal enveloping algebra. Let $\mathfrak{X}$ be the centralizer of $K$ in $\mathfrak{G}$, so we have a projection

$$
D \mapsto D_{K}=\int_{K} \operatorname{Ad} k \cdot D d k: \mathfrak{G} \rightarrow \mathfrak{X} .
$$

Essentially, a spherical function is a continuous representation $\Phi: I_{c \mathrm{~d}}(G) \rightarrow$ $\mathscr{L}(F)$ on a finite dimensional space $F$ satisfying $\Phi(u)=1 \in \mathscr{L}(F)$ for some $u \in I_{c o}(G)$. Such a representation is given by integration against a function $\Phi: G \rightarrow \mathscr{L}(F)$. Namely

$$
\Phi(f)=\int_{G} f(x) \Phi(x) d x \quad\left(f \in I_{c \mathrm{~d}}(G)\right)
$$

where $\Phi$ is given by

$$
\Phi(x)=\Phi\left((x \cdot u)^{\circ}\right) \quad \text { where } x \cdot u(y)=u\left(x^{-1} y\right) .
$$

Since the main interest is in irreducible representations of $I_{c \mathrm{~d}}(G)$, we include that in the definition [W2, p. 14].

DEFINITION. A spherical function of type $\mathfrak{d} \in \hat{K}$ is a continuous function $\Phi: G \rightarrow \mathscr{L}(F)$ (where $F$ is a finite dimensional complex vector space) satisfying

(1) $\Phi$ is quasi-bounded [W2, p. 6],

(2) $\Phi\left(k x k^{-1}\right)=\Phi(x)$ for all $k \in K$ and $x \in G$,

(3) $\bar{\alpha}_{\mathfrak{D}} * \Phi=\Phi$ (where $\bar{\alpha}_{\mathfrak{D}}$ is the complex conjugate),

(4) $f \mapsto \Phi(f)=\int_{G} f(x) \Phi(x) d x$ is an irreducible representation of $I_{c o}(G)$ on $F$. Two spherical functions are equivalent, written $\left[\Phi_{1}\right]=\left[\Phi_{2}\right]$, if there is a linear isomorphism $Q: F_{1} \rightarrow F_{2}$ such that $Q \Phi_{1}(x)=\Phi_{2}(x) Q$ for all $x \in G$ (iff the corresponding representations of $I_{c \nu}(G)$ are equivalent $)$. Let $\left[I_{c 0}(G)\right]$ be the set of equivalence classes of spherical functions of type $\mathfrak{d}$.

See [W2, around p. 14] for the general properties of spherical functions. We recall that spherical functions come from representations of $G$.

DEFINITION. Let $\pi, E$ be a representation of $G$ with $[\pi: \mathfrak{d}]<\infty($ where $\mathfrak{d} \in \hat{K})$. Define

$$
F_{\pi \mid \mathfrak{d}}=\operatorname{Hom}_{K}\left(E_{\mathfrak{0}}, E\right) \quad \text { and } \quad \Phi(\pi, \mathfrak{d}): G \rightarrow \mathscr{L}\left(F_{\pi \mid d}\right)
$$

by

$$
\Phi(\pi, \mathfrak{d}, x) \cdot l=\int_{K} \pi\left(k x k^{-1}\right) \circ l d k \quad\left(l \in F_{\pi \mid \mathfrak{o}}\right) .
$$

PROPOSITION 2. $\Phi(\pi, \mathfrak{d})$ satisfies (2) and (3) in the definition of spherical function, and defines a representation of $I_{c D}(G)$ which is irreducible when $\pi$ is TCI and quasi-bounded when $E$ is a Banach space. Conversely if $\Phi: G \rightarrow \mathscr{L}(F)$ satisfies (2), (3) and (4) except for the irreducibility, then there exists an FDS [W1, p. 231] representation $\pi, E$ of $G$ such that $\Phi(\pi, \mathfrak{d})$ is equivalent to $\Phi$. Moreover $\pi, E$ satisfies the following universal property: If $\pi_{1}, E_{1}$ is any FDS representation with $\left[\pi_{1}: \mathfrak{d}\right]<\infty$ and $E_{1}$ is cyclic with respect to $P(\mathfrak{d}) \cdot E_{1}$ and $Q_{\mathfrak{0}}: F_{\pi_{1} \mid \mathfrak{o}} \rightarrow F$ intertwines $\Phi\left(\pi_{1}, \mathfrak{d}\right)$ and $\Phi$, then there exists a unique Naimark intertwining operator $Q: E_{1} \rightarrow E$ extending $Q_{\mathfrak{0}}$. When $\Phi$ is irreducible then $\pi, E$ is TCI and when $\Phi$ is quasi-bounded we may take $E$ to be a Banach space.

Let $[G](\mathfrak{d})$ be the set of Naimark equivalence classes $[\pi]$ of TCI (Banach) representations of $G$ such that $[\pi: \mathfrak{d}]>0$ (finite). Then [W2, p. 13]. 
COROLlaRY 3. $[\pi] \mapsto[\Phi(\pi, \mathfrak{d})]$ is well defined and gives a one-to-one correspondence between $[G](\mathfrak{d})$ and $\left[I_{c \mathcal{O}}(G)\right]$.

REMARK. The proposition remains true when you eliminate references to Banach spaces and the quasi-boundedness assumption from the definition of spherical function. We will show below that quasi-boundedness is a consequence of the other properties of spherical functions when $G$ is a Cartan motion group (or a semisimple group). This will answer affirmatively a conjecture of Fell: that any TCI representation of $G$ on a locally convex space is Naimark equivalence to a TCI representation on a Banach space.

Let us state explicitly the following

COROLlARY 4. Let $\pi_{1}: G \rightarrow \mathscr{L}\left(E_{1}\right)$ be an admissible representation and let $\pi: G \rightarrow \mathscr{L}(E)$ be TCI. Suppose $[\pi: \mathfrak{d}]>0$. Suppose that the (irreducible) spherical function $\Phi(\pi, \mathfrak{d})$ is equivalent to an irreducible factor in the Jordan-Hölder series for the representation $f \mapsto \Phi\left(\pi_{1}, \mathfrak{d}, f\right)$. Then $\pi, E$ is Naimark equivalent to an irreducible factor in the Jordan-Hölder series for $\pi_{1}, E_{1}$. (See [W1, p. 333].)

Next, we recall the definition of the Fell topology on $[G]$ and $\left[I_{c \mathrm{~d}}(G)\right]$. For $[\pi] \in[G]$, let $\mathscr{A}(\pi)$ be the space of functions of the form $\lambda(x)=\operatorname{tr}(T \pi(x))$ where $T \in \mathscr{L}(E)$ is left and right $K$-finite. Then a net $\left[\pi_{n}\right]$ converges to $[\pi]$ in $[G]$ if and only if for all $\lambda \in \mathscr{A}(\pi)$ there exist $\lambda_{n} \in \mathscr{A}\left(\pi_{n}\right)$ such that $\lambda_{n}(f)$ converges to $\lambda(f)$ for all $f \in C_{c}(G)$. Since $\pi$ is TCI, it is the same to say there exists $\lambda \in \mathscr{A}(\pi)$, $\lambda \neq 0$, and $\lambda_{n} \in \mathscr{A}\left(\pi_{n}\right)$ such that $\lambda_{n}$ converges to $\lambda$ uniformly on compacta. The Fell topology on $\left[I_{c \mathcal{O}}(G)\right]$ is defined analogously. The following is easy.

PROPOSITION 5. The $[G](\mathfrak{d})$ form an open covering of $[G]$ and the map $[\pi] \mapsto$ $[\Phi(\pi, \mathfrak{d})]$ defines a homeomorphism of $[G](\mathfrak{d})$ onto $\left[I_{c \mathfrak{d}}(G)\right]$.

A similar result holds for the space $\hat{G}$ of unitary equivalence classes of irreducible unitary representations of $G$. Recall that $\hat{G} \subseteq[G][\mathbf{W} 1$, p. 245] and that the Fell topology on $\hat{G}$ agrees with the hull-kernel topology. Let $\left[I_{* \mathrm{~d}}(G)\right]$ be the closure of $\left[I_{c \mathcal{D}}(G)\right]$ in the $C^{*}$ algebra of $G$, and let $I_{* \mathcal{D}}(G)^{\wedge}$ be the space of unitary equivalence classes of irreducible continuous *-representations with the hull-kernel topology. Then again $I_{* \mathrm{~d}}(G)^{\wedge}$ is naturally a subspace of $\left[I_{* \mathrm{~d}}(G)\right]$, see [W1, p. 245].

PROPOSITION 6. The $\hat{G}(\mathfrak{d})$ form an open covering of $\hat{G}$ and $\hat{\pi} \mapsto \hat{\Phi}(\pi, \mathfrak{d})$ gives a homeomorphism of $\hat{G}(\mathfrak{d})$ onto $I_{* \mathfrak{d}}(G)^{\wedge}$. Moreover the Plancherel measures (which exist for abstract reasons) are mutually determined by

$$
d \mu_{\mathfrak{o}}=d(\mathfrak{d}) d \mu \mid \hat{G}(\mathfrak{d})
$$

This is folklore except for the surjectivity of $\hat{\pi} \mapsto \hat{\Phi}(\pi, \mathfrak{d})$; use $[\mathbf{S}$, p. 17] and [D, pp. 22, 25].

Now assume that $G$ is a connected Lie group and $K$ is a maximal compact subgroup which is large in $G$. Let $\mathfrak{g}$ be its Lie algebra and $\mathfrak{G}$ be its (complex) universal enveloping algebra. If $D \in \mathfrak{G}$ and $f \in C^{\infty}(G)$, let $f(x ; D)$ denote the left invariant derivative $D f$ evaluated at $x \in G$. Let $\mathfrak{X}$ be the centralizer of $K$ in $\mathfrak{G}$, and recall the projection $D \mapsto D_{K}$.

The next proposition, essentially due to Godement [G, p. 540], is the main tool used for analyzing spherical functions. The proof can be obtained by jiggling [W2, p. 19] a little, or in [R] in exactly this form. 
PROPOSITION 7. Let $F$ be a finite dimensional complex vector space and let $\Phi: G \rightarrow \mathscr{L}(F)$ be real analytic. Suppose that $\Phi(1)=1$ and $\Phi(1 ; D)=\Phi\left(1 ; D_{K}\right)$ for $D \in \mathfrak{G}$. Suppose that $\Phi\left(x ; D_{K}\right)=\Phi(x) \Phi\left(1 ; D_{K}\right)$ for all $D_{K} \in \mathfrak{X}$ and that $D_{K} \mapsto \Phi\left(1 ; D_{K}\right): \mathfrak{X} \rightarrow \mathscr{L}(F)$ is an irreducible representation of the algebra $\mathfrak{X}$. Then, if $\Phi$ is quasi-bounded, then $\Phi$ is a spherical function of type $\mathfrak{d}$ for some $\mathfrak{d} \in \hat{K}$. Conversely, a spherical function of type $\mathfrak{d}$ has these properties. (The proposition remains true if all references to quasi-boundedness are eliminated.)

REMARK 8. Let us record the form that $\Phi(\pi, \mathfrak{d})$ takes for induced representations $\pi$. Let $P$ be a closed subgroup of $G$ such that $G=K P$ and set $K_{P}=K \cap$ $P$. Let $\delta_{G}$ and $\delta_{P}$ be the left modular functions for $G$ and $P$ respectively (so if $d x$ is left invariant Haar measure on $G$ then $\left.d\left(x^{-1}\right)=\delta_{G}(x) d x\right)$. Let $P^{+}$be the set of continuous group homomorphisms $\Lambda: P \rightarrow \mathbf{C}$ (the additive group of complex numbers). In the compact-open topology, $P^{+}$is a Fréchet vector space, which is locally compact, hence finite dimensional, if $P$ is compactly generated $\bmod \bigcap\left\{\operatorname{ker} \Lambda \mid \Lambda \in P^{+}\right\}$. Define $\rho \in P^{+}$by $\rho(p)=\frac{1}{2} \log \left(\delta_{P}(p) / \delta_{G}(p)\right)$.

Now let $\sigma: P \rightarrow \mathscr{L}\left(E_{\sigma}\right)$ be a representation of $P$ on a Hilbert space $E_{\sigma}$ which is admissible with respect to $K_{P}$. Let $\pi=\pi(P, \sigma, \Lambda)$ be the induced representation of $G$, realized by left translation on the space $E$ of $L_{2}$ functions $e: K \rightarrow E_{\sigma}$ which extend to $G$ so that

$$
e(k p)=e^{-(\Lambda+\rho)(p)} \sigma\left(p^{-1}\right) \cdot e(k) \quad(e \in E) .
$$

From [W 1, 5.5.1.4] it follows that the $\pi(f)$ are realized as integral operators

$$
\begin{aligned}
& \pi(f) \cdot e\left(k_{1}\right)=\int_{K} \pi(f)\left(k_{1}, k_{2}\right) e\left(k_{2}^{-1}\right) d k_{2}, \\
& \pi(f)\left(k_{1}, k_{2}\right)=\int_{P} f\left(k_{1} p k_{2}\right) e^{(\Lambda-\rho) p} \sigma(p) d p
\end{aligned}
$$

for $f \in C_{c}(G)$. By Frobenius reciprocity

$$
F_{\pi \mid \mathfrak{0}}=\operatorname{Hom}_{K}\left(E_{\mathfrak{0}}, E\right) \simeq \operatorname{Hom}_{K_{P}}\left(E_{\mathfrak{0}}, E_{\sigma}\right) .
$$

For $k \in K$ and $p \in P$, define an endomorphism of this last space by

$$
l \mapsto \int_{K_{P}} \sigma(m p) \circ l \circ \mu_{\mathfrak{o}}\left(k m^{-1}\right) d m \quad\left(l \in \operatorname{Hom}_{K_{P}}\left(E_{\mathfrak{0}}, E_{\sigma}\right)\right) .
$$

Abusing notation, write this as $\sigma(p) \circ l \circ \mu_{\mathfrak{o}}(k)$. For $x \in G$ choose $\kappa(x) \in K$ and $p(x) \in P$ so that $x=\kappa(x) p(x)$. (Usually $\kappa\left(m x m^{-1}\right)=m \kappa(x) m^{-1}$ and similarly for $p(x)$, so the integration over $K_{P}$ above is unnecessary.) Then the induced (reducible) spherical function is given by

$$
\Phi(\pi, \mathfrak{d}, x) \cdot l=\int_{K} \sigma\left(p\left(k^{-1} x k\right)\right) \circ l \circ \mu_{\mathfrak{o}}\left(\kappa\left(k^{-1} x k\right)\right) e^{(\Lambda-\rho) p(x k)} d k .
$$

(You can see this by integrating against $f \in I_{c \mathcal{0}}(G)$, using the integral operator above.) Note that the integrand does not depend on the choice $\kappa(x)$ and $p(x)$.

When $G$ is a Lie group, the derivatives of these induced spherical functions can also be computed. Let $\mathfrak{G}, \mathfrak{K}, \mathfrak{P}$ and $\mathfrak{K}_{P}$ be the respective universal enveloping algebras, so $\mathfrak{G}=\mathfrak{K} \otimes_{\mathfrak{K}_{P}} \mathfrak{P}$. For $D_{K} \in \mathfrak{X}$ write

$$
D_{K}=\sum_{n} D_{k n} D_{p n} \quad\left(D_{k n} \in \mathfrak{K}, D_{p n} \in \mathfrak{P}\right) \text {. }
$$


We may assume

$$
\sum_{n} \operatorname{Ad} m \cdot D_{k n} \otimes \operatorname{Ad} m \cdot D_{p n}=\sum_{n} D_{k n} \otimes D_{p n}
$$

for $m \in K_{P}$. Then

$$
\Phi\left(\pi, \mathfrak{o}, 1 ; D_{K}\right) \cdot l=\sum_{n}\left(e^{\Lambda-\rho} \sigma\right)\left(1 ; D_{p n}\right) \circ l \circ \mu_{\mathfrak{o}}\left(1 ; D_{k n}\right) .
$$

(This is well defined by the assumed $K_{P}$-invariance.)

Finally we shall also require the analogue of Harish-Chandra's Eisenstein integrals in the context of Cartan motion groups, so let us review the definition. Let $\tau=\left(\tau_{1}, \tau_{2}\right)$ be a double unitary representation of $K$ on the finite dimensional Hilbert space $V$; that is

$$
\left(k_{1}, k_{2}\right), v \mapsto \tau_{1}\left(k_{1}\right) v \tau_{2}\left(k_{2}^{-1}\right): K \times K \rightarrow \mathscr{L}(V)
$$

defines a unitary representation of $K \times K$. Let $\pi=\pi(P, \sigma, \Lambda)$ be the induced representation on $E$ as above. Then $\mathscr{L}(E)$ becomes a double $K$-module by left and right composition. Let $T: \mathscr{L}(E) \rightarrow V$ be a homomorphism of double $K$-modules. Since $\mathscr{L}(E)$ densely contains the $K \times K$-module induced from the $K_{P} \times K_{P^{-}}$ module $\mathscr{L}\left(E_{\sigma}\right)$ (and $V$ is finite dimensional), we have the Frobenius reciprocity isomorphism $T \mapsto \psi_{T}=T(1,1)$ between the space of double $K$-homomorphisms $T: \mathscr{L}(E) \rightarrow V$ and the space of double $K_{P}$-homomorphisms $\psi_{T}: \mathscr{L}\left(E_{\sigma}\right) \rightarrow V$. Now chasing through the identifications gives

$$
\begin{aligned}
T(\pi(x)) & =E\left(P, \sigma, \Lambda, \psi_{T}, x\right) \\
& =\int_{K} \tau_{1}(\kappa(x k)) \psi_{T}(\sigma(p(x k))) \tau_{2}\left(k^{-1}\right) e^{(\Lambda-\rho) p(x k)} d k
\end{aligned}
$$

(Use [W1, p. 448] again and $T\left(k_{1}, k_{2}\right)=\tau_{1}\left(k_{1}\right) \psi_{T} \tau_{2}\left(k_{2}\right)$, the inverse of the Frobenius reciprocity isomorphism.) This is Harish-Chandra's Eisenstein integral [H-CI, p. 150]. (Note that the integrand is independent of the arbitrary choice made in writing $x=\kappa(x) p(x)$.)

2. Prelude to the main result. Let $G$ be a connected reductive Lie group, let $\mathfrak{g}$ be its Lie algebra and let $\bar{G}$ be the analytic subgroup corresponding to $[\mathfrak{g}, \mathfrak{g}$ ]. Assume $\bar{G}$ has finite center. Let $K$ be a maximal compact subgroup of $G$ and let $\mathfrak{g}=\mathfrak{k}+\mathfrak{s}$ be the corresponding Cartan decomposition. Then $K$ acts on $\mathfrak{s}$ by $k \cdot x=\operatorname{Ad} k \cdot x$. The Cartan motion group $H$ (associated to $G$ ) is the semidirect product $H=K \times \mathfrak{s}$; thus

$$
\left(k_{1}, x_{1}\right)\left(k_{2}, x_{2}\right)=\left(k_{1} k_{2}, \operatorname{Ad} k_{2}^{-1} \cdot x_{1}+x_{2}\right) .
$$

According to a theorem of Godement [W1, p. 314], $K$ is large in $H$, and in fact if $\pi$ is a TCI representation of $H$ and $\mathfrak{d} \in \hat{K}$, then $\mathfrak{d}$ occurs in $\pi$ no more than $d(\mathfrak{d})$ times (i.e. $\left.\operatorname{dim}\left(F_{\pi \mid \mathfrak{d}}\right) \leq d(\mathfrak{d})\right)$. Thus the abstractions of the first section apply to $H$. First we wish to parameterize $\left[I_{c \mathrm{~d}}(H)\right]$. Proposition 7 indicates that it will be helpful to find the irreducible representations of $\mathfrak{X}$, and so, in this section and the next, we will derive results on the structure of $\mathfrak{X}$.

Let $\mathfrak{h}$ be the Lie algebra of the Cartan motion group $H$ and let $\mathfrak{a}_{\mathfrak{s}}$ be a Cartan subspace of $\mathfrak{s}$. If $\mu_{\mathfrak{0}}: K \rightarrow \mathscr{L}\left(E_{\mathfrak{0}}\right)(\mathfrak{o}$ in $\hat{K})$ is an irreducible representation, let 
$\mathfrak{K}_{\mathfrak{0}}$ be the kernel of the corresponding representation of the universal enveloping algebra $\mathfrak{K}$ (so $\mathfrak{K} / \mathfrak{K}_{\mathfrak{0}}$ is isomorphic to $\mathscr{L}\left(E_{\mathfrak{0}}\right)$ as a $\mathfrak{K}$-module). If $\Phi$ is a spherical function of type $\mathfrak{o}$, then $\Phi\left(D_{k} D\right)=\Phi\left(1 ; D_{k} D\right)=0$ (for $D_{k} \in \mathfrak{K}_{\mathfrak{d}}$ and $D \in \mathfrak{H}$ ). Hence $\Phi$ kills $\left\{D_{K} \mid D \in \mathfrak{K}_{\mathfrak{0}} \mathfrak{H}\right\}=\mathfrak{K}_{\mathfrak{0}} \mathfrak{H} \cap \mathfrak{X}$, which is a two sided ideal in $\mathfrak{X}$. As an Ad $K$-module, we may identify

$$
\mathfrak{K}_{\mathfrak{0}} \mathfrak{H} \backslash \mathfrak{H}=\mathfrak{K}_{\mathfrak{d}} \backslash \mathfrak{K} \otimes S(\mathfrak{s})=\mathscr{L}\left(E_{\mathfrak{0}}\right) \otimes S(\mathfrak{s})
$$

(where $S(\mathfrak{s})$ is the symmetric algebra of $\mathfrak{s}$ ). Then $\mathfrak{X} / \mathfrak{K}_{\mathfrak{0}} \mathfrak{H} \cap \mathfrak{X}$ is identified with the space of $K$-invariants:

$$
\begin{aligned}
& \sum_{n} L_{n} \otimes D_{s n}=\sum_{n} \mu_{\mathfrak{o}}(k) L_{n} \mu_{\mathfrak{o}}\left(k^{-1}\right) \otimes \operatorname{Ad} k \cdot D_{s n} \\
& \qquad\left(k \in K, L_{n} \in \mathscr{L}\left(E_{\mathfrak{J}}\right), D_{s n} \in S(\mathfrak{s})\right) .
\end{aligned}
$$

More generally, let $\tau=\left(\tau_{1}, \tau_{2}\right)$ be a unitary double representation of $K$ on a finite dimensional complex Hilbert space $V$. Let $S(\mathfrak{s}, \tau)$ be the space of $K$-invariants in $V \otimes S(\mathfrak{s})$

$$
\sum_{n} v_{n} \otimes D_{s n}=\sum_{n} \tau_{1}(k) v_{n} \tau_{2}\left(k^{-1}\right) \otimes \operatorname{Ad} k \cdot D_{s n}
$$

$$
\left(k \in K, v_{n} \in V, D_{s n} \in S(\mathfrak{s})\right) .
$$

Fix a Cartan-Killing form $B$ which is positive definite on $\mathfrak{s}$ and negative definite on $\mathfrak{k}$. Use $B$ to identify elements of $S(\mathfrak{s})$ with polynomial functions on $\mathfrak{s}$. Thus $S(\mathfrak{s}, \tau)$ becomes the space of polynomial functions $f: \mathfrak{s}_{c} \rightarrow V$ satisfying

$$
f(\operatorname{Ad} k \cdot x)=\tau_{1}(k) f(x) \tau_{2}\left(k^{-1}\right) \quad\left(x \in \mathfrak{s}_{c}, k \in K\right) .
$$

Let $\mathfrak{z}$ be the orthogonal complement of $\mathfrak{a}_{\mathfrak{s}}$ in $\mathfrak{s}$.

DEFINITION. Define the linear map $\beta(\tau, \cdot): S(\mathfrak{s}, \tau) \rightarrow V \otimes S\left(\mathfrak{a}_{s}\right)$ by

$$
\beta\left(\tau, \sum v_{n} \otimes D_{s n}\right)=\sum v_{n} \otimes D_{a n} \quad \text { where } D_{s n}-D_{a n} \in S(\mathfrak{s}) \mathfrak{z} .
$$

In terms of polynomial functions this is just the operation of restriction, $f \mapsto f \mid \mathfrak{a}_{\mathfrak{s}}$. Also define $\beta_{\mathfrak{b}}: \mathfrak{X} \rightarrow \mathscr{L}\left(E_{\mathfrak{b}}\right) \otimes S\left(\mathfrak{a}_{\mathfrak{s}}\right)$ as the composition of the natural map

$$
\mathfrak{X} \rightarrow \mathfrak{X} / \mathfrak{K}_{\mathfrak{d}} \mathfrak{H} \cap \mathfrak{X}=S(\mathfrak{s}, \tau) \text { and } \beta(\tau): S(\mathfrak{s}, \tau) \rightarrow \mathscr{L}\left(E_{\mathfrak{d}}\right) \otimes S\left(\mathfrak{a}_{\mathfrak{s}}\right) \text {. }
$$

(Here $V=\mathscr{L}\left(E_{\mathfrak{0}}\right)$ with its obvious double $K$-module structure.)

Let $W_{\mathfrak{s}}=M^{*} / M$ be the Weyl group of $\left(\mathfrak{g}, \mathfrak{a}_{\mathfrak{s}}\right)$, where $M^{*}$ is the normalizer of $\mathfrak{a}_{\mathfrak{s}}$ in $K$ and $M$ is the centralizer. Also fix an Iwasawa decomposition $\mathfrak{g}=\mathfrak{k}+\mathfrak{a}_{\mathfrak{s}}+\mathfrak{n}$, and let $\Sigma_{+}$be the corresponding set of positive (restricted) roots of the pair $\left(\mathfrak{g}, \mathfrak{a}_{\mathfrak{s}}\right)$. Define

$$
\pi=\prod\left\{\lambda \mid \lambda \in \Sigma_{+} \text {and } \frac{1}{2} \lambda \notin \Sigma_{+}\right\}
$$

so $\pi^{2} \in S\left(\mathfrak{a}_{\mathfrak{s}}\right)$ is invariant under $W_{\mathfrak{s}}$. Let $\mathfrak{a}_{\mathfrak{s} c}$ be the complexification of $\mathfrak{a}_{\mathfrak{s}}$.

DEFINITION. Let $S\left(\mathfrak{a}_{\mathfrak{s} c}, \tau\right)$ be the space of polynomial functions $f: \mathfrak{a}_{\mathfrak{s} c} \rightarrow V$ satisfying

(1) If $\Lambda \in \mathfrak{a}_{\mathfrak{s} c}$ and $k \in K$ and $\operatorname{Ad} k \cdot \Lambda=\Lambda$ then $\tau_{1}(k) f(\Lambda) \tau_{2}\left(k^{-1}\right)=f(\Lambda)$

(2) If $\Lambda \in \mathfrak{a}_{\mathfrak{s} c}$ and $w \in W_{\mathfrak{s}}$ then $f(w \cdot \Lambda)=\tau_{1}(m) f(\Lambda) \tau_{2}\left(m^{-1}\right)$ (where $m \in M^{*}$ is a representative of $w$ ).

(Note that by condition 1 the right-hand side in (2) is independent of the choice of $m \in M^{*}$ representing $w$.) We now have the following (very weak) version of Chevalley's restriction theorem [H, p. 430]. 
LEMMA 11. $\beta(\tau): S(\mathfrak{s}, \tau) \rightarrow V \otimes S\left(\mathfrak{a}_{\mathfrak{s}}\right)$ is one-to-one and preserves degrees, and its image satisfies $S\left(\mathfrak{a}_{\mathfrak{s} c}, \tau\right) \supseteq \beta(\tau, S(\mathfrak{s}, \tau)) \supseteq S\left(\mathfrak{a}_{\mathfrak{s} c}, \tau\right) \cdot \pi^{2 n}$ (for some sufficiently large integer $n)$. Moreover $\beta_{\mathfrak{0}}: \mathfrak{X} \rightarrow \mathscr{L}\left(E_{\mathfrak{0}}\right) \otimes S\left(\mathfrak{a}_{\mathfrak{s}}\right)$ is an algebra antihomomorphism.

ProOF. Since $\mathfrak{s}=\left\{\operatorname{Ad} k \cdot H \mid k \in K\right.$ and $\left.H \in \mathfrak{a}_{\mathfrak{s}}\right\}$, and by analyticity, it is clear that $\beta(\tau)$ is one to one and preserves degrees and its image lies in $S\left(\mathfrak{a}_{\mathfrak{s} c}, \tau\right)$. Let $D_{K} \in \mathfrak{X}$ and $E \in \mathfrak{H}$ and write

$$
D_{K}=\sum D_{k l} D_{a l}+D_{z} \text { and } E=\sum E_{k n} E_{a n}+E_{z}
$$

where $D_{k l}, E_{k n} \in \mathfrak{K}$ and $D_{a l}, E_{a n} \in S\left(\mathfrak{a}_{\mathfrak{s}}\right)$ and $D_{z}$ and $E_{z} \in \mathfrak{H z}$. Then

$$
\begin{aligned}
D_{K} E & =\sum_{n} E_{k n} D_{K} E_{a n}+D_{K} E_{z} \\
& =\sum_{l, n} E_{k n} D_{k l} D_{a l} E_{a n} \quad(\bmod \mathfrak{H z}) .
\end{aligned}
$$

Thus

$$
\beta_{\mathfrak{d}}\left(D_{K} E\right)=\sum_{l, n} \mu_{\mathfrak{d}}\left(E_{k n}\right) \mu_{\mathfrak{d}}\left(D_{k l}\right) \otimes E_{a n} D_{a l}=\beta_{\mathfrak{d}}(E) \beta_{\mathfrak{d}}\left(D_{K}\right)
$$

(where of course the multiplication on $\mathscr{L}\left(E_{\mathfrak{0}}\right) \otimes S\left(\mathfrak{a}_{\mathfrak{s}}\right)$ is the tensor product of that on $\mathscr{L}\left(E_{\mathfrak{0}}\right)$ with that on $\left.S\left(\mathfrak{a}_{\mathfrak{s}}\right)\right)$.

In order to prove the second inclusion, we complexify everything in sight. Let $K_{c} \supseteq K$ be a connected complex Lie group with Lie algebra $\mathfrak{k}_{c}$, and let $\mathfrak{s}_{c}$ and $\mathfrak{a}_{\mathfrak{s} c}$ be the complexifications of $\mathfrak{s}$ and $\mathfrak{a}_{\mathfrak{s}}$ respectively. Extend $\left(\tau_{1}, \tau_{2}\right)$ and Ad to holomorphic (hence algebraic) representations of $K_{c}$. If $M_{c}$ and $M_{c}^{*}$ denote the centralizer and normalizer of $\mathfrak{a}_{\mathfrak{s} c}$ in $K_{c}$, and $M_{c}^{\circ}$ is the identity component of $M_{c}$, then $M_{c}=M M_{c}^{\circ}$ and $M_{c}^{*}=M^{*} M_{c}^{\circ}$ (by $[\mathbf{H}$, p. 244] and [W1, p. 86]). Thus if $f \in S\left(\mathfrak{a}_{\mathfrak{s} c}, \tau\right)$ and $\Lambda \in \mathfrak{a}_{\mathfrak{s} c}$ and $m \in M_{c}^{*}$, then

$$
f(\operatorname{Ad} m \cdot \Lambda)=\tau_{1}(m) f(\Lambda) \tau_{2}\left(m^{-1}\right) .
$$

Let $\tilde{\pi}^{2}: \mathfrak{s}_{c} \rightarrow \mathbf{C}$ be the $\operatorname{Ad}\left(K_{c}\right)$-invariant polynomial function corresponding to the $W_{\mathfrak{s}}$-invariant polynomial $\pi^{2}$ on $\mathfrak{a}_{\mathfrak{s c}}\left[\mathbf{H}\right.$, p. 430]. Let $\mathfrak{s}_{c}^{\prime}$ be the Zariski open set of nonzeros of $\tilde{\pi}^{2}$ in $\mathfrak{s}_{c}$ and set $\mathfrak{a}_{\mathfrak{s} c}^{\prime}=\mathfrak{a}_{\mathfrak{s} c} \cap \mathfrak{s}_{c}^{\prime}$. Then $\mathfrak{s}_{c}^{\prime}$ is the set of $s$-regular elements in $\mathfrak{s}_{c}$ and hence each element of $\mathfrak{s}_{c}^{\prime}$ is conjugate by $K_{c}$ to an element of $\mathfrak{a}_{\mathfrak{s} c}^{\prime}$ [K\&R, p. 764]. Thus we have an algebraic morphism $(k, \Lambda) \mapsto \operatorname{Ad} k \cdot \Lambda: K_{c} / M_{c} \times \mathfrak{a}_{s c}^{\prime} \rightarrow \mathfrak{s}_{c}^{\prime}$ where two points have the same image exactly when they are conjugate under the (free) action of $W_{\mathfrak{s}}$ by right translation on $K_{c} / M_{c}$ and the usual action on $\mathfrak{a}_{\mathfrak{s} c}^{\prime}$.

As observed above, any $f \in S\left(\mathfrak{a}_{\mathfrak{a} c}, \tau\right)$ is equivariant under $M_{c}^{*}$, and hence can be extended to a rational morphism $\tilde{f}: \mathfrak{s}_{c}^{\prime} \rightarrow V$ by the formula

$$
\tilde{f}(\operatorname{Ad} k \cdot \Lambda)=\tau_{1}(k) f(\Lambda) \tau_{2}\left(k^{-1}\right) \quad\left(\operatorname{Ad} k \cdot \Lambda \in \mathfrak{s}_{c}^{\prime}\right) .
$$

Thus, clearing denominators, we may extend $\pi^{2 n} f \in S\left(\mathfrak{a}_{\mathfrak{s c}}, \tau\right)$ to a polynomial function $\tilde{\pi}^{2 n} \tilde{f} \in S\left(\mathfrak{s}_{c}, \tau\right)$ ( $n$ depending on $f$ ). But since $S\left(\mathfrak{a}_{\mathfrak{s} c}, \tau\right)$ is a finitely generated module over the (Noetherian) ring of $W_{\mathfrak{s}}$-invariants in $S\left(\mathfrak{a}_{\mathfrak{s} c}\right)[\mathbf{W} \mathbf{1}, \mathrm{p}$. 147], we can choose one integer $n$ such that $\tilde{\pi}^{2 n}$ clears all denominators. For this $n, \beta(\tau, S(\mathfrak{s}, \tau)) \supseteq \pi^{2 n} \cdot S\left(\mathfrak{a}_{\mathfrak{s c}}, \tau\right)$.

The proof of this lemma shows that every element of $S\left(\mathfrak{a}_{\mathfrak{s c}}, \tau\right)$ extends to a rational function $\mathfrak{s}_{c}^{\prime} \rightarrow V$; the point of the second inclusion is that it gives us some hold on the denominators (since $\pi$ is a product of distinct primes). 
COROLLARY 12. The kernel of the algebra antihomomorphism $\beta_{\mathfrak{d}}: \mathfrak{X} \rightarrow \mathscr{L}\left(E_{\mathfrak{d}}\right)$ $\otimes S\left(\mathfrak{a}_{\mathfrak{s}}\right)$ is precisely $\mathfrak{K}_{\mathfrak{d}} \mathfrak{H} \cap \mathfrak{X}$. Also if $\Phi: H \rightarrow \mathscr{L}(F)$ is a spherical function of type $\mathfrak{d}$ then there is a unique irreducible anti-representation $\Phi_{1}: \beta_{\mathfrak{o}}(\mathfrak{X}) \rightarrow \mathscr{L}(F)$ such that

$$
\Phi\left(1 ; D_{K}\right)=\Phi_{1} \circ \beta_{\mathfrak{o}}\left(D_{K}\right) \quad\left(\text { for all } D_{K} \in \mathfrak{X}\right) \text {. }
$$

This is clear from the definition of $\beta_{\mathfrak{0}}$ and Lemma 11 and Proposition 7.

Let $I(\mathfrak{s})$ be the algebra of Ad $K$-invariants in $S(\mathfrak{s})$, so $I(\mathfrak{s})$ is a (Noetherian) subalgebra of the center of $\mathfrak{X}$ (in fact it is central in $\mathfrak{H}$.) By Chevalley's restriction theorem, $\beta_{\mathfrak{o}}$ takes $I(\mathfrak{s})$ isomorphically onto $1 \otimes I\left(\mathfrak{a}_{\mathfrak{s}}\right)$, where $I\left(\mathfrak{a}_{\mathfrak{s}}\right) \subseteq S\left(\mathfrak{a}_{\mathfrak{s}}\right)$ is the subalgebra of $W_{\mathfrak{s}}$-invariants. If $\Phi$ is an (irreducible) spherical function of type $\mathfrak{d}$ then $I(\mathfrak{s})$ is represented by scalars, say

$$
\Phi\left(1 ; D_{K}\right)=\eta\left(D_{K}\right) \cdot 1 \text { for } D_{K} \in I(\mathfrak{s}) \text {. }
$$

Thus to parameterize the spherical functions of type $\mathfrak{d}$, we must parameterize the irreducible anti-representations of $\beta_{\mathfrak{d}}(\mathfrak{X})$ which represent $1 \otimes I\left(\mathfrak{a}_{\mathfrak{s}}\right)$ by scalars. But the one dimensional representations of $I\left(\mathfrak{a}_{\mathfrak{s}}\right)$ are of the form $D_{a} \mapsto e^{\Lambda}\left(D_{a}\right)$ where $\Lambda: \mathfrak{a}_{\mathfrak{s}} \rightarrow \mathbf{C}$ is real linear and $e^{\Lambda}$ is the corresponding representation of $S\left(\mathfrak{a}_{\mathfrak{s}}\right)$.

Let $\Lambda$ also denote the extension to $\mathfrak{s}$ so that $\mathfrak{z} \subseteq$ ker $\Lambda$, and let $K_{\Lambda}=\{k \in$ $K \mid \operatorname{Ad} k \cdot \Lambda=\Lambda\}$. Let $V=\mathscr{L}\left(E_{\mathfrak{0}}\right)$ and $\tau$ be as in the definition of $\beta_{\mathfrak{o}}$. Then by the definition of $S\left(\mathfrak{a}_{\mathfrak{s c}}, \tau\right) \supseteq \beta_{\mathfrak{d}}(\mathfrak{X})$ we have

$$
\left(1 \otimes e^{\Lambda}\right) \circ \beta_{\mathfrak{d}}(\mathfrak{X}) \subseteq\left\{L \in \mathscr{L}\left(E_{\mathfrak{d}}\right) \mid \mu_{\mathfrak{d}}(k) \circ L=L \circ \mu_{\mathfrak{d}}(k) \text { for } k \in K_{\Lambda}\right\} .
$$

LEMMA 13. Let $\Phi$ be a spherical function of type $\mathfrak{d} \in \hat{K}$, and suppose the corresponding representation $\Phi: \mathfrak{X} \rightarrow \mathscr{L}(F)$ satisfies $\Phi(D)=\eta(D) \cdot 1$ for all $D \in$ $I(\mathfrak{s})$. Let $\Lambda: \mathfrak{a}_{\mathfrak{s}} \rightarrow \mathbf{C}$ be a real-linear function such that $\eta=\left(1 \otimes e^{\Lambda}\right) \circ \beta_{\mathfrak{d}}$ on $I(\mathfrak{s})$. Then there exists an $\mathrm{e} \in \hat{K}_{\Lambda}$ such that $\Phi$ is equivalent to an irreducible factor in the Jordan-Hölder series for

$$
\Phi(\mathfrak{e}, \Lambda, \mathfrak{d}): \mathfrak{X} \rightarrow \mathscr{L}\left(F_{\mathfrak{d} \mid \mathfrak{e}}\right) \quad\left(\text { where } F_{\mathfrak{o} \mid \mathfrak{e}}=\operatorname{Hom}_{K_{\Lambda}}\left(E_{\mathfrak{o}}, E_{\mathfrak{e}}\right)\right)
$$

given by

$$
\Phi(\mathfrak{e}, \Lambda, \mathfrak{d}, D) \cdot l=\sum_{n}\left(l \circ L_{n}\right) e^{\Lambda}\left(D_{a n}\right)
$$

where $l \in F_{\mathfrak{\partial} \mid \mathrm{e}}$ and $D \in \mathfrak{X}$ and

$$
\beta_{\mathfrak{o}}(D)=\sum_{n} L_{n} \otimes D_{a n} \in \mathscr{L}\left(E_{\mathfrak{j}}\right) \otimes S\left(\mathfrak{a}_{\mathfrak{s}}\right)
$$

Moreover, $\Phi\left(\mathfrak{e}_{1}, \Lambda_{1}, \mathfrak{d}\right)$ and $\Phi\left(\mathfrak{e}_{2}, \Lambda_{2}, \mathfrak{d}\right)$ are disjoint (i.e., their Jordan-Hölder series contain no common factors) unless $\Lambda_{1}=w \Lambda_{2}$ for some $w \in W_{\mathrm{s}}$.

PROOF. First note that by the remarks preceding the statement of the lemma, $\Phi(\mathfrak{e}, \Lambda, \mathfrak{d})$ is well defined. We seek the irreducible anti-representations of $\beta_{\mathfrak{d}}(\mathfrak{X})$ (Corollary 12). From [W1, p. 147], $\mathscr{L}\left(E_{\mathfrak{d}}\right) \otimes S\left(\mathfrak{a}_{\mathfrak{s}}\right)$ is finitely generated as a $1 \otimes$ $I\left(\mathfrak{a}_{\mathfrak{s}}\right)$ module, so the hypotheses of the first three lemmas in the appendix hold for $1 \otimes I\left(\mathfrak{a}_{\mathfrak{s}}\right) \subseteq \beta_{\mathfrak{o}}(\mathfrak{X}) \subseteq \mathscr{L}\left(E_{\mathfrak{l}}\right) \otimes S\left(\mathfrak{a}_{\mathfrak{s}}\right)$. (These are easily translated for antirepresentations.)

Now the irreducible anti-representations of $\mathscr{L}\left(E_{\mathfrak{0}}\right) \otimes S\left(\mathfrak{a}_{\mathfrak{s}}\right)$ are of the form

$$
\Phi_{\Lambda}\left(L \otimes D_{a}\right) \cdot l=l \circ L \cdot e^{\Lambda}\left(D_{a}\right) \quad\left(l \in E_{\mathfrak{d}}^{\vee}\right) .
$$


From Lemma 11 , the image of $\beta_{\mathfrak{v}}(\mathfrak{X})$ under $\left(1 \otimes e^{\Lambda}\right)$ is contained in the centralizer of $\mu_{\mathfrak{o}}\left(K_{\Lambda}\right)$ in $\mathscr{L}\left(E_{\mathfrak{o}}\right)$, so we may further decompose $\Phi_{\Lambda} \mid \beta_{\mathfrak{\jmath}}(\mathfrak{X})$. In particular, every irreducible factor in the Jordan-Hölder series for $\Phi_{\Lambda} \mid \beta_{\mathfrak{v}}(\mathfrak{X})$ appears in the JordanHölder series for $\Phi(\mathfrak{e}, \Lambda, \mathfrak{d})$ for some $\mathfrak{e} \in \hat{K}_{\Lambda}$. The first statement of the lemma now follows from the third lemma of the appendix, and the last statement from $[\mathbf{H}, \mathrm{p}$. 433].

It will turn out that the $\Phi(\mathfrak{e}, \Lambda, \mathfrak{d})$ are already irreducible, so Lemma 13 gives a parametrization of $\left[I_{c \mathcal{O}}(H)\right]$. However, we will need a much better description of the images of $\beta(\tau)$ and $\beta_{\mathfrak{b}}$ in order to show this. For now, using Lemma 13, we can prove a subquotient theorem for $H$ (analogous to a theorem of Harish-Chandra for semisimple groups [W1, p. 452]). This lemma also answers in the affirmative, for Cartan motion groups, a conjecture of Fell [F2, p. 287].

LEMMA 14. Let $\pi: H \rightarrow \mathscr{L}(E)$ be a TCI representation of $H$ on a complete locally convex space $E$. Then there is a quasi-character $e^{\Lambda}: \mathfrak{s} \rightarrow \mathbf{C}^{\times}$such that $\mathfrak{z} \subseteq \operatorname{ker} \Lambda$ and an $\mathfrak{e} \in \hat{K}_{\Lambda}$ such that $\pi$ is Naimark equivalent to an irreducible factor in the Jordan-Hölder series of the representation $\pi(\mathfrak{e}, \Lambda)$ of $H$ induced from $(k, x) \mapsto \mu_{\mathfrak{e}}(k) e^{\Lambda x}: K_{\Lambda} \times \mathfrak{s} \rightarrow \mathscr{L}\left(E_{\mathfrak{e}}\right)$. In particular $\pi$ is Naimark equivalent to a TCI representation on a Hilbert space.

ProOF. First, as to notation, $K_{\Lambda} \times \mathfrak{s}$ is to be taken as a subgroup of $H$. Then, since $\operatorname{Ad} k \cdot \Lambda=\Lambda$ for $k \in K_{\Lambda}$, we see that the formula above really does define a representation of $K_{\Lambda} \times \mathbf{s}$.

Choose $\mathfrak{d} \in \hat{K}$ which occurs in $\pi$ with (finite) positive multiplicity, and let $\Phi(\pi, \mathfrak{d})$ be the corresponding spherical function (without the quasi-boundedness assumption). Then Lemma 13 says that $\Phi(\pi, \mathfrak{d})$ occurs in the Jordan-Hölder series of $\Phi(\mathfrak{e}, \Lambda, \mathfrak{d})$ for some $\Lambda$ and $\mathfrak{e}$. Compare the formula in Lemma 13 for $\Phi(\mathfrak{e}, \Lambda, \mathfrak{d}, D)$ with formula (9) for an induced spherical function (here $\sigma=\mu_{e} e^{\Lambda}$ and the modular functions are trivial). We see that $\Phi(\mathfrak{e}, \Lambda, \mathfrak{d})$ is just the (reducible) spherical function of type $\mathfrak{d}$ occurring in the representation induced from $\mu_{\mathrm{e}} e^{\Lambda}$. Since $H$ is connected, $\mathfrak{X}$ is dense in the compactly supported $K$-central distributions (with respect to the weak topology generated by the real analytic functions). Thus we see that $\Phi(\pi, \mathfrak{d})$ occurs in the Jordan-Hölder series for the representation $\Phi(\pi(\mathfrak{e}, \Lambda), \mathfrak{d})$ of $I_{c \mathfrak{d}}(H)$. So now Corollary 4 tells us that $\pi$ is Naimark equivalent to an irreducible subquotient of $\pi(\mathfrak{e}, \Lambda)$ (a subquotient of the Hilbert space $L_{2}\left(K, E_{\mathfrak{e}}\right)$ ).

All of the results we have obtained so far (and their proofs) go through for connected semisimple Lie groups too (see $[\mathbf{L}$ or $\mathbf{R}]$ ). However, for Cartan motion groups we can actually say much more. We can actually parameterize $\left[I_{c o}(H)\right]$, which is to say, we can decompose the $\Phi(\mathfrak{e}, \Lambda, \mathfrak{d})$ and find the equivalences among the factors. In order to do this, however, we will need much more precise information on the image of $\beta(\tau)$ (see Lemma 11), and this is the objective of the next section.

3. The main result. The goal of this section is to compute the image $\beta_{\mathfrak{d}}(\mathfrak{X}) \subseteq$ $\mathscr{L}\left(E_{\mathfrak{0}}\right) \otimes S\left(\mathfrak{a}_{\mathfrak{s}}\right)$ and more generally the image of $\beta(\tau)$. This is an extension of Chevalley's restriction theorem. First we will obtain the result for Cartan motion groups corresponding to rank one semisimple groups. For this we will need the next result on the radial component of the Laplace operator $\omega_{\mathfrak{s}}$ (see [W2, p. 277]).

Let $G$ be a reductive group with Lie algebra $\mathfrak{g}$ and fix an Iwasawa decomposition $\mathfrak{g}=\mathfrak{k}+\mathfrak{a}_{\mathfrak{s}}+\mathfrak{n}$. Let $\mathfrak{n}=\sum_{\lambda} \mathfrak{n}^{\lambda}+\mathfrak{n}^{2 \lambda}$ be an eigenspace decomposition under the 
adjoint action of $\mathfrak{a}_{\mathfrak{s}}$ and put $m(\lambda)=\operatorname{dim} \mathfrak{n}^{\lambda}$ and $m(2 \lambda)=\operatorname{dim} \mathfrak{n}^{2 \lambda}$. If $X \in \mathfrak{n}$, write $X=Y+Z$ where $Y \in \mathfrak{k}$ and $Z \in \mathfrak{s}$, and set $\mathfrak{y}_{\lambda}=\left\{Y \mid X \in \mathfrak{n}^{\lambda}\right\}$ and $\mathfrak{y}_{2 \lambda}=\{Y \mid X \in$ $\left.\mathfrak{n}^{2 \lambda}\right\}$. Defining $\mathfrak{k}_{\lambda}=\mathfrak{m}+\mathfrak{y}_{\lambda}+\mathfrak{y}_{2 \lambda}$ and $\mathfrak{k}_{2 \lambda}=\mathfrak{m}+\mathfrak{y}_{2 \lambda}$ (where $\mathfrak{m}$ is the Lie algebra of $M$, the centralizer of $\mathfrak{a}_{\mathfrak{s}}$ in $K$ ), we see that $\mathfrak{k}_{\lambda}$ and $\mathfrak{k}_{2 \lambda}$ are subalgebras of $\mathfrak{k}$.

Let $\mathfrak{a}_{c}$ be a $\theta$-stable Cartan subalgebra of the complexification $\mathfrak{g}_{c}$ containing $\mathfrak{a}_{\mathfrak{s}}$. Let $\Phi^{+}$be the set of positive roots of $\left(\mathfrak{g}_{c}, \mathfrak{a}_{c}\right)$ with respect to an ordering compatible with the given Iwasawa decomposition, and let $P^{+}$be the set of $\alpha \in \Phi^{+}$such that $\alpha \mid \mathfrak{a}_{\mathfrak{s}} \neq 0$. Let $X_{ \pm \alpha}$ be the corresponding Weyl basis [W1, p. 4]. For $\alpha \in P^{+}$, write $X_{ \pm \alpha}=Y_{ \pm \alpha}+Z_{ \pm \alpha}$ where $Y_{ \pm \alpha} \in \mathfrak{k}_{c}$ and $Z_{ \pm \alpha} \in \mathfrak{s}_{c}$. Define $H_{\lambda} \in \mathfrak{a}_{\mathfrak{s}}$ by $B\left(H_{\lambda}, h\right)=\lambda(h)=\alpha(h)$ (where $h \in \mathfrak{a}_{\mathfrak{s}}$ and $\alpha \mid \mathfrak{a}_{\mathfrak{s}}=\lambda$, and $B$ is a nondegenerate Killing form on $\mathfrak{g})$. Define the $\operatorname{Ad}(K)$-invariant differential operator $\omega_{\mathfrak{s}} \in S\left(\mathfrak{s}_{c}\right)$ by

$$
\omega_{\mathfrak{s}}=\sum_{i} H_{i}^{2}+2 \sum_{\alpha \in P^{+}} Z_{\alpha} Z_{-\alpha}
$$

(where $H_{i}$ is an orthonormal basis for $\mathfrak{a}_{\mathfrak{s}}$ with respect to $B$ ). Define

$$
\tilde{\omega}_{\lambda}=\sum_{\alpha \mid a_{s}=\lambda} Y_{\alpha} Y_{-\alpha}+Y_{-\alpha} Y_{\alpha}
$$

and define $\tilde{\omega}_{2 \lambda}$ similarly. Set

$$
\omega_{\lambda}=\omega_{\mathfrak{m}}+\tilde{\omega}_{\lambda}+\tilde{\omega}_{2 \lambda} \text { and } \omega_{2 \lambda}=\omega_{\mathfrak{m}}+\tilde{\omega}_{2 \lambda}
$$

where $\omega_{\mathfrak{m}}$ is the Casimir operator of $\mathfrak{m}$ computed relative to $B$. Also note that when $G$ is semisimple,

$$
\sum_{i} H_{i}^{2}=\sum_{\lambda}(2 m(\lambda)+8 m(2 \lambda)) H_{\lambda}^{2}
$$

(because the two sides agree on homogeneous quadratics on $\mathfrak{a}_{\mathfrak{s}}$ ).

Let $V, \tau=\left(\tau_{1}, \tau_{2}\right)$ be a unitary double representation of $K$, and define the unitary (single) representation $\mu: K \rightarrow \mathscr{L}(V)$ by $\mu(k) \cdot v=\tau_{1}(k) \cdot v \cdot \tau_{2}\left(k^{-1}\right)$. Extend $\mu$ to $\mathfrak{K}$ as usual. Let $\mathfrak{s}^{\prime}$ be the set of $s$-regular elements of $\mathfrak{s}$.

LEMMA 15. Let $f: \mathfrak{s}^{\prime} \rightarrow V$ be a differentiable function and suppose for all $k \in K$ and $x \in \mathfrak{s}^{\prime} \subseteq H$ that $f\left(k x k^{-1}\right)=\tau_{1}(k) f(x) \tau_{2}\left(k^{-1}\right)$. Let $a \in \mathfrak{a}_{\mathfrak{s}}^{\prime}=\mathfrak{a}_{\mathfrak{s}} \cap \mathfrak{s}^{\prime}$. Then

$$
\begin{aligned}
f\left(a ; \omega_{\mathfrak{s}}\right)= & f\left(a ; \sum_{i} H_{i}^{2}\right)+f\left(a ; \sum_{\lambda}(m(\lambda)+m(2 \lambda)) \lambda(a)^{-1} H_{\lambda}\right) \\
& -\sum_{\lambda} \lambda(a)^{-2}\left(\mu\left(\omega_{\lambda}\right)-\frac{3}{4} \mu\left(\omega_{2 \lambda}\right)\right) \cdot f(a)
\end{aligned}
$$

(where the sum is over the positive short restricted roots $\Sigma_{+}^{1}$ of $\left(\mathfrak{g}, \mathfrak{a}_{\mathfrak{s}}\right)$ ). Write $\delta\left(\omega_{\mathfrak{s}}\right)$ for the differential operator appearing on the right in the above equation.

ProOF. If $a \in \mathfrak{a}_{\mathfrak{s}} \subseteq H$ then

$$
\left(\operatorname{Ad} a^{-1}-1\right) Y_{ \pm \alpha}=-\frac{1}{2}\left[a, X_{ \pm \alpha}+\theta X_{ \pm \alpha}\right]=\mp \alpha(a) Z_{ \pm \alpha}
$$

Thus we have

$$
\mu\left(Y_{ \pm \alpha}\right) f(a)=\tau_{1}\left(Y_{ \pm \alpha}\right) f(a)-f(a) \tau_{2}\left(Y_{ \pm \alpha}\right)=f\left(a ; \mp \alpha(a) Z_{ \pm \alpha}\right)
$$


and

$$
\begin{aligned}
\mu\left(Y_{-\alpha} Y_{\alpha}\right) f(a) & =f\left(a ; \operatorname{Ad} a^{-1} Y_{-\alpha}\left(-\alpha(a) Z_{\alpha}\right)-\left(-\alpha(a) Z_{\alpha}\right) Y_{-\alpha}\right) \\
& =f\left(a ;-\alpha(a)^{2} Z_{-\alpha} Z_{\alpha}-\alpha(a)\left[Y_{-\alpha}, Z_{\alpha}\right]\right) \\
\mu\left(Y_{\alpha} Y_{-\alpha}\right) f(a) & =f\left(a ;-\alpha(a)^{2} Z_{\alpha} Z_{-\alpha}+\alpha(a)\left[Y_{\alpha}, Z_{-\alpha}\right]\right) .
\end{aligned}
$$

Now $H_{\beta}=\left[Y_{\alpha}, Z_{-\alpha}\right]+\left[Z_{\alpha}, Y_{-\alpha}\right] \in \mathfrak{a}_{\mathfrak{s}} \subseteq \mathfrak{h}$ satisfies $B\left(H_{\beta}, h\right)=\beta(h)$ (for all $h \in \mathfrak{a}_{\mathfrak{s}}$ ) where $\beta=\alpha \mid \mathfrak{a}_{\mathfrak{s}}=\lambda$ or $2 \lambda$ for some $\lambda$. Thus we obtain

$$
\begin{aligned}
f\left(a ; \omega_{\mathfrak{s}}\right) & =f\left(a ; \sum H_{i}^{2}+2 \sum_{\alpha \in P^{+}} Z_{\alpha} Z_{-\alpha}\right) \\
& =f\left(a ; \sum H_{i}^{2}+\sum_{P^{+}} \beta(a)^{-1} H_{\beta}\right)-\sum_{P^{+}} \beta(a)^{-2} \mu\left(Y_{\alpha} Y_{-\alpha}+Y_{-\alpha} Y_{\alpha}\right) \cdot f(a) .
\end{aligned}
$$

The result now follows from the remarks preceding the lemma, and the observation that $\mu\left(\omega_{\mathrm{m}}\right) \cdot f(a)=0$ so that

$$
\left(\mu\left(\tilde{\omega}_{\lambda}\right)+\frac{1}{4} \mu\left(\tilde{\omega}_{2 \lambda}\right)\right) \cdot f(a)=\left(\mu\left(\omega_{\lambda}\right)-\frac{3}{4} \mu\left(\omega_{2 \lambda}\right)\right) \cdot f(a) .
$$

Now, retaining the above notation, let $K_{2 \lambda}$ be the smallest subgroup of $K$ containing $M$ and with Lie algebra $\mathfrak{k}_{2 \lambda}$, and define $K_{\lambda}$ similarly. If $\mathfrak{d}_{\lambda} \in \hat{K}_{\lambda}$, let $P\left(\mathfrak{d}_{\lambda}\right): V \rightarrow V$ be the orthogonal projection onto the space of vectors in $V$ which transform according to $\mathfrak{d}_{\lambda}$ under $\mu$, and define $\left|\mathfrak{d}_{\lambda}\right|^{2} \in \mathbf{R}$ by the equation $\mu\left(\omega_{\lambda}\right) P\left(\mathfrak{d}_{\lambda}\right)=\left|\mathfrak{d}_{\lambda}\right|^{2} P\left(\mathfrak{d}_{\lambda}\right)$. Define $P\left(\mathfrak{d}_{2 \lambda}\right)$ and $\left|\mathfrak{d}_{2 \lambda}\right|^{2}$ similarly, for $\mathfrak{d}_{2 \lambda} \in \hat{K}_{2 \lambda}$. Note that the space $V_{M}$ of $\mu(M)$-invariant vectors in $V$ is stable under $P\left(\mathfrak{d}_{\lambda}\right)$ and $P\left(\mathfrak{d}_{2 \lambda}\right)$. In the next lemma we assume that the reductive group corresponding to $H$ is of rank $=1$ (i.e., $\operatorname{dim} \mathfrak{a}_{\mathfrak{s}}=1$ ), so $K_{\lambda}=K$.

LEMMA 16. Let $H$ be a Cartan motion group such that $\operatorname{dim} \mathfrak{a}_{\mathfrak{s}}=1$. Let $\mathfrak{d}_{\lambda} \in$ $\hat{K}_{\lambda}$ and $\mathfrak{d}_{2 \lambda} \in \hat{K}_{2 \lambda}$ and suppose $P\left(\mathfrak{d}_{\lambda}\right) P\left(\mathfrak{d}_{2 \lambda}\right) \mid V_{M} \neq 0$. Then there is a (unique) nonnegative integer solution to the equation

$$
k^{2}+(m(\lambda)+m(2 \lambda)-1) k=(2 m(\lambda)+8 m(2 \lambda))\left(\left|\mathfrak{d}_{\lambda}\right|^{2}-\frac{3}{4}\left|\mathfrak{d}_{2 \lambda}\right|^{2}\right)
$$

(where $\left|\mathfrak{d}_{2 \lambda}\right|^{2}=0$ if $\left.m(2 \lambda)=0\right)$. Writing $k=k\left(\mathfrak{d}_{\lambda}, \mathfrak{d}_{2 \lambda}\right)$ for this solution, define the endomorphism $R$ of $V_{M} \otimes S\left(\mathfrak{a}_{\mathfrak{s}}\right)$ by

$$
R=\sum P\left(\mathfrak{d}_{\lambda}\right) P\left(\mathfrak{d}_{2 \lambda}\right) \otimes \lambda^{k}
$$

(where the sum is over those $\mathfrak{d}_{\lambda} \in \hat{K}_{\lambda}$ and $\mathfrak{d}_{2 \lambda} \in \hat{K}_{2 \lambda}$ such that $P\left(\mathfrak{d}_{\lambda}\right) P\left(\mathfrak{d}_{2 \lambda}\right) \mid V_{M} \neq$ 0 , and $\lambda^{k}$ is the operator of multiplication by $\lambda^{k}$ in $\left.S\left(\mathfrak{a}_{\mathfrak{s}}\right)\right)$. Then the inage of $\beta(\tau): S(\mathfrak{s}, \tau) \rightarrow V \otimes S\left(\mathfrak{a}_{\mathfrak{s}}\right)$ is precisely equal to the space of $W_{\mathfrak{s}}$-invariants in the image $R\left(V_{M} \otimes S\left(\mathfrak{a}_{\mathfrak{s}}\right)\right)$. In fact $R$ is an isomorphism of $I\left(\mathfrak{a}_{\mathfrak{s}}\right)$-algebras from $V_{M} \otimes \mathbf{C}$ $I\left(\mathfrak{a}_{\mathfrak{s}}\right)$ onto the image of $\beta(\tau)$.

ProOF. (1) Let us observe that for a polynomial function $f \in S(\mathfrak{s}, \tau), f\left(a ; \delta\left(\omega_{\mathfrak{s}}\right)^{n}\right)$ $=\omega_{\mathfrak{s}}^{n} \cdot f(a)$ defined on $\mathfrak{a}_{\mathfrak{s}}^{\prime}$ extends to an element of $S\left(\mathfrak{a}_{\mathfrak{s}}, \tau\right)$ and for $n>\frac{1}{2} \operatorname{deg} f=$ $\frac{1}{2} \operatorname{deg}\left(f \mid \mathfrak{a}_{\mathfrak{s}}\right)$ we have $f\left(a ; \delta\left(\omega_{\mathfrak{s}}\right)^{n}\right)=0$ (for all $a \in \mathfrak{a}_{\mathfrak{s}}^{\prime}$ ). But we also have a converse statement. Let $f: \mathfrak{s}^{\prime} \rightarrow V$ be a rational function on $\mathfrak{s}$ which is everywhere defined on $\mathfrak{s}^{\prime}$ and satisfies $f\left(k x k^{-1}\right)=\tau_{1}(k) f(x) \tau_{2}\left(k^{-1}\right)$ for $k \in K$ and $x \in \mathfrak{s}^{\prime} \subseteq H$. Suppose that $f \mid \mathfrak{a}_{\mathfrak{s}} \in S\left(\mathfrak{a}_{\mathfrak{s}}, \tau\right)$ and that $f\left(\cdot ; \delta\left(\omega_{\mathfrak{s}}\right)^{n}\right)$ is locally bounded on $\mathfrak{a}_{\mathfrak{s}}$ for every positive 
integer $n$. Then I claim that $f$ is actually a polynomial function and $f \in S(\mathfrak{s}, \tau)$. Let us first prove this.

(2) Clearly $f\left(\cdot ; \delta\left(\omega_{\mathfrak{s}}\right)^{n}\right)$ has a rational extension to $\mathfrak{s}^{\prime}$, namely $\omega_{\mathfrak{s}}^{n} \cdot f$. We show that the assumption that $f\left(\cdot ; \delta\left(\omega_{\mathfrak{s}}\right)^{n}\right)$ is locally bounded implies that $\omega_{\mathfrak{s}}^{n} \cdot f$ is locally bounded on $\mathfrak{s}$. Thus let $x \in \mathfrak{s}$ and select a compact neighborhood $U$ of $x$ in $\mathfrak{s}$ which is invariant under $\operatorname{Ad}(K)$. Then the set of values assumed by $\omega_{\mathfrak{s}}^{n} \cdot f$ on $U \cap \mathfrak{s}^{\prime}$ has the same bound as the set of values assumed on $U \cap \mathfrak{a}_{\mathfrak{s}}^{\prime}$ by $\omega_{\mathfrak{s}}^{n} \cdot f \mid \mathfrak{a}_{\mathfrak{s}}=f\left(\cdot ; \delta\left(\omega_{\mathfrak{s}}\right)^{n}\right)$ (because $\omega_{\mathfrak{s}}^{n}$ commutes with the $\mu(k)$ and $\mu$ is unitary). Moreover, this bound is finite since $U \cap \mathfrak{a}_{\mathfrak{s}}$ is compact. Thus $\omega_{\mathfrak{s}}^{n} \cdot f$ (defined on $\mathfrak{s}^{\prime}$ ) is locally bounded on $\mathfrak{s}$.

(3) Next we show that $f\left(\cdot ; \delta\left(\omega_{\mathfrak{s}}\right)^{n}\right)=0$ for $n>\frac{1}{2} \operatorname{deg}\left(f \mid \mathfrak{a}_{\mathfrak{s}}\right)$. Recall the polynomial function $\pi^{2}=\prod \lambda^{2}$ (product over $\lambda \in \Sigma_{+}^{1}$ ) used in the proof of Lemma 11. From the formula for $\delta\left(\omega_{\mathfrak{s}}\right)$ given in Lemma 15 we see that $\pi^{2} \delta\left(\omega_{\mathfrak{s}}\right)$ is a differential operator with polynomial coefficients defined on $\mathfrak{a}_{\mathfrak{s}}$. Thus $f\left(\cdot ; \pi^{2} \delta\left(\omega_{\mathfrak{s}}\right)\right)$ is a polynomial function on $\mathfrak{a}_{\mathfrak{s}}$. But by assumption $f\left(\cdot ; \delta\left(\omega_{\mathfrak{s}}\right)\right)=f\left(\cdot ; \pi^{2} \delta\left(\omega_{\mathfrak{s}}\right)\right) / \pi^{2}$ is locally bounded on $\mathfrak{a}_{\mathfrak{s}}$, and this implies that the numerator is divisible by $\pi^{2}$ (since $\pi^{2}$ is a product of real valued linear functions). Thus $f\left(\cdot ; \delta\left(\omega_{\mathrm{s}}\right)\right)$ is a polynomial function on $\mathfrak{a}_{\mathfrak{s}}$, and it clearly has degree $\leq \operatorname{deg}\left(f \mid \mathfrak{a}_{\mathfrak{s}}\right)-2$. By induction, $f\left(\cdot ; \delta\left(\omega_{\mathfrak{s}}\right)^{n}\right)$ is a polynomial function of degree $\leq \operatorname{deg}\left(f \mid \mathfrak{a}_{\mathfrak{s}}\right)-2 n$, and so if $n>\frac{1}{2} \operatorname{deg}\left(f \mid \mathfrak{a}_{\mathfrak{s}}\right)$ then $f\left(\cdot ; \delta\left(\omega_{\mathfrak{s}}\right)^{n}\right)=0$. (Induction is valid here because we only use the facts that $f \mid \mathfrak{a}_{\mathfrak{s}}$ is a polynomial function and $f\left(\cdot ; \delta\left(\omega_{\mathfrak{s}}\right)^{n}\right)$ is locally bounded; we do not need the fact that $f \mid \mathfrak{a}_{\mathfrak{s}} \in S\left(\mathfrak{a}_{\mathfrak{s}}, \tau\right)$.)

(4) From steps (2) and (3) we see that for sufficiently large $n, \omega_{\mathfrak{s}}^{n} \cdot f=0$ on $\mathfrak{s}$ in the sense of distributions. But $\omega_{\mathrm{s}}^{n}$ is an elliptic differential operator and so $f$ is actually a real-analytic function defined on all of $\mathfrak{s}$. Next we compute its Taylor series at the origin.

(5) Choose any element $X \in \mathfrak{s} \subseteq \mathfrak{h}$ and let $n$ be an integer $n>\operatorname{deg}\left(f \mid \mathfrak{a}_{\mathfrak{s}}\right)$. Choose $k \in K$ and $H \in \mathfrak{a}_{\mathfrak{s}} \subseteq \mathfrak{h}$ such that $\operatorname{Ad} k \cdot H=X$. But then

$$
f\left(0 ; X^{n}\right)=\mu(k) \cdot f\left(0 ; H^{n}\right)=\mu(k) \cdot\left(f \mid \mathfrak{a}_{\mathfrak{s}}\right)\left(0 ; H^{n}\right)=0 .
$$

Since the $X^{n}$ (as $X$ varies in $\mathfrak{s}$ ) span the space of homogeneous differential operators of degree $n$, we see that the homogeneous terms in the Taylor series of $f$ of degree $>\operatorname{deg}\left(f \mid \mathfrak{a}_{\mathfrak{s}}\right)$ all vanish. This implies that $f$ is a polynomial function, $f \in S(\mathfrak{s}, \tau)$.

(6) Let $w_{\lambda} \in W_{\mathrm{s}}$ be the reflection in the root plane corresponding to $\lambda$, and assume $m(2 \lambda) \neq 0$. Then it is not hard to see that $K_{2 \lambda}$ contains a representative of $w_{\lambda}$. (Consider the reductive analytic group $G_{2 \lambda} \subseteq G$ corresponding to $\mathfrak{m}+\mathfrak{a}_{\mathfrak{s}}+$ $\mathfrak{n}^{2 \lambda}+\mathfrak{n}^{-2 \lambda}$, so $K_{2 \lambda}^{\circ}=K \cap G_{2 \lambda}$.)

(7) So far we have not invoked the rank one assumption; we do so now. Let $\mathfrak{d}_{\lambda} \in \hat{K}_{\lambda}$ and $\mathfrak{d}_{2 \lambda} \in \hat{K}_{2 \lambda}$ and suppose $P\left(\mathfrak{d}_{\lambda}\right) P\left(\mathfrak{d}_{2 \lambda}\right) \mid V_{M} \neq 0$. Select a nonzero $\nu \in V_{M}$ such that $P\left(\mathfrak{d}_{\lambda}\right) P\left(\mathfrak{d}_{2 \lambda}\right) \cdot \nu=\nu$. By step (6), since $w \in K_{2 \lambda}$, we may also assume that $\mu(w) \cdot \nu= \pm \nu$ for $w \in W_{\mathfrak{s}}$. Call $\nu$ even if $\mu(w) \cdot \nu=\nu$ and odd if $\mu(w) \cdot \nu=-\nu$ (where $w$ is the nontrivial element of $W_{\mathfrak{s}}$ ). Let us show that $\nu \otimes S\left(\mathfrak{a}_{\mathfrak{s}}\right)$ intersects the image of $\beta(\tau)$. Clearly the $W_{\mathfrak{s}}$-invariant polynomial functions in $\nu \otimes S\left(\mathfrak{a}_{\mathfrak{s}}\right)$ are exactly those of the form $\nu \cdot \sum c_{m} \lambda^{m}$ where $c_{m}=0$ unless the parity of $m$ agrees with the parity of $\nu$. Also if $k \in K$ and $a \in \mathfrak{a}_{\mathfrak{s}}$ and $\operatorname{Ad} k \cdot a=a$ then either $k \in M$ or $a=0$. Thus if the polynomial function $f=\nu \cdot \sum c_{m} \lambda^{m}$ has no constant term, or if $\mathfrak{d}_{\lambda}$ is the trivial representation, then $f(a)=\tau_{1}(k) f(a) \tau_{2}\left(k^{-1}\right)$ whenever Ad $k \cdot a=a$. Thus by Lemma 11 we may choose 
a sufficiently large integer $n$ such that $\nu \cdot \lambda^{2 n} \sum c_{m} \lambda^{m}$ lies in the image of $\beta(\tau)$ whenever $\nu \cdot \sum c_{m} \lambda^{m}$ satisfies both of the above conditions.

The classical Chevalley restriction theorem [H, p. 430] says that the restriction of $I(\mathfrak{s})$, the $K$-invariants in $S(\mathfrak{s})$, consists of the even polynomials on $\mathfrak{a}_{\mathfrak{s}}$. Since $S(\mathfrak{s}, \tau)$ is a module over $I(\mathfrak{s})$, the image of $\beta(\tau)$ is a module over the even polynomials. Thus there is a nonnegative integer $k=k\left(\mathfrak{d}_{\lambda}, \mathfrak{d}_{2 \lambda}\right)$ such that $\nu \otimes S\left(\mathfrak{a}_{\mathfrak{s}}\right) \cap$ image $\beta(\tau)$ consists precisely of the polynomial functions $\nu \cdot \sum c_{m} \lambda^{m}$ where the sum is over a finite set of integers $m \geq k\left(\mathfrak{D}_{\lambda}, \mathfrak{d}_{2 \lambda}\right)$ with the parity of $m$ equal to the parity of $\nu$. Note that $k\left(\mathfrak{d}_{\lambda}, \mathfrak{d}_{2 \lambda}\right)$ is zero if $\mathfrak{d}_{\lambda}$ is trivial, and is greater than zero when $\mathfrak{d}_{\lambda}$ is nontrivial (because then $\nu$ is not $\mu(K)$-invariant so $\nu \cdot c_{0} \notin S\left(\mathfrak{a}_{\mathfrak{s}}, \tau\right)$ ).

(8) We have yet to show that the nonnegative integer $k=k\left(\mathfrak{d}_{\lambda}, \mathfrak{d}_{2 \lambda}\right)$ found in step (7) satisfies the quadratic equation in the statement of the lemma. We note here a few properties of this quadratic. Observe that the coefficient of $k, m(\lambda)+m(2 \lambda)-1$, is nonnegative, so the quadratic has at most one nonnegative root. Also we show that the right-hand side is nonnegative, and positive if $\boldsymbol{D}_{\lambda}$ is nontrivial, so that the quadratic does have a nonnegative root which is positive if $\mathfrak{d}_{\lambda}$ is nontrivial.

To see this, observe that

$$
\left|\mathfrak{d}_{\lambda}\right|^{2}=B\left(\Lambda+2 \rho_{\lambda}, \Lambda\right) \quad \text { and } \quad\left|\mathfrak{d}_{2 \lambda}\right|^{2}=B\left(\Lambda_{2}+2 \rho_{2 \lambda}, \Lambda_{2}\right)
$$

where $\Lambda$ is the highest weight of $\mathfrak{d}_{\lambda} \in \hat{K}$ and $2 \rho_{\lambda}$ is the sum of the positive roots of $K$, and similarly for $\Lambda_{2}$ and $2 \rho_{2 \lambda}$ on $K_{2 \lambda}$ (and $B$ is the fixed Killing form on $\mathfrak{g}$ ). In particular $\left|\mathfrak{d}_{\lambda}\right|^{2}>0$ if $\mathfrak{d}_{\lambda}$ is nontrivial, so we are done if $m(2 \lambda)=0$.

So suppose $m(2 \lambda) \neq 0$; then $m(2 \lambda)$ is odd so there is a real root $\alpha$ of $\left(\mathfrak{g}_{c}, \mathfrak{a}_{c}\right)$ (where $\mathfrak{a}_{c} \supseteq \mathfrak{a}_{\mathfrak{s}}$ ) such that $\alpha \mid \mathfrak{a}_{\mathfrak{s}}=2 \lambda$ [W1, p. 33], and $K_{2 \lambda}$ contains a Cartan subgroup of $G$ and of $K$. Let $\Lambda, \Lambda_{2}, \rho_{\lambda}$ and $\rho_{2 \lambda}$ of the preceding paragraph refer to this Cartan subalgebra (with a fixed ordering). Assuming that $P\left(\mathfrak{d}_{\lambda}\right) P\left(\mathfrak{d}_{2 \lambda}\right) \mid V_{M} \neq$ 0 , it follows that $\mathfrak{d}_{2 \lambda}$ occurs in $\mathfrak{d}_{\lambda}$ with positive multiplicity, and so $\Lambda_{2}$ is also a weight of $\mathfrak{d}_{\lambda}$. This implies that $B\left(\Lambda_{2}, \Lambda_{2}\right) \leq B(\Lambda, \Lambda)$ and that $\Lambda-\Lambda_{2}$ is a nonnegative integral linear combination of positive roots of $K$. Also $\rho_{\lambda}$ is a highest weight of $\mathfrak{k}$, hence of $\mathfrak{k}_{2 \lambda}$, so $\rho_{\lambda}$ is dominant integral for $\mathfrak{k}_{2 \lambda}$. We may assume that the ordering is chosen so that a simple root $\alpha$ of $\mathfrak{k}_{2 \lambda}$ is positive for $\mathfrak{k}$. Then $B\left(\rho_{\lambda}, \alpha\right)>0$ so

$$
2 B\left(\rho_{\lambda}-\rho_{2 \lambda}, \alpha\right) / B(\alpha, \alpha)=2 B\left(\rho_{\lambda}, \alpha\right) / B(\alpha, \alpha)-1 \geq 0 .
$$

Hence $\rho_{\lambda}-\rho_{2 \lambda}$ is dominant on $\mathfrak{k}_{2 \lambda}$ and so

$$
B\left(\Lambda_{2}+2 \rho_{2 \lambda}, \Lambda_{2}\right) \leq B\left(\Lambda_{2}, \Lambda_{2}\right)+B\left(2 \rho_{\lambda}, \Lambda_{2}\right) \leq B(\Lambda, \Lambda)+B\left(2 \rho_{\lambda}, \Lambda\right) .
$$

Thus we see that $\left|\mathfrak{d}_{2 \lambda}\right|^{2} \leq\left|\mathfrak{d}_{\lambda}\right|^{2}$ and so $\left|\mathfrak{d}_{\lambda}\right|^{2}-\frac{3}{4}\left|\mathfrak{d}_{2 \lambda}\right|^{2} \geq \frac{1}{4}\left|\mathfrak{d}_{\lambda}\right|^{2}>0$ if $\mathfrak{d}_{\lambda}$ is nontrivial.

(9) Let us now show that the nonnegative integer $k=k\left(\mathfrak{d}_{\lambda}, \mathfrak{d}_{2 \lambda}\right)$ found in step (7) satisfies the quadratic equation in the statement of the lemma. So let $v \in V_{M}$, $v \neq 0$, and suppose that $P\left(\mathfrak{d}_{\lambda}\right) P\left(\mathfrak{d}_{2 \lambda}\right) \cdot v=v$ and $\mu(w) \cdot v= \pm v\left(w \in W_{\mathbf{s}}\right.$ nontrivial). Let $f=v \cdot \sum c_{m} \lambda^{m}$ be any polynomial function in $v \otimes S\left(\mathfrak{a}_{\mathfrak{s}}\right)$. Step (1) suggests that it might be helpful to look for the condition that $f\left(\cdot ; \delta\left(\omega_{s}\right)^{m}\right)$ be locally bounded on $\mathfrak{a}_{\mathfrak{s}}$ for arbitrarily large $m$, and by step (3), this is the same as requesting that it be a polynomial function. So let us compute $f\left(\cdot ; \delta\left(\omega_{s}\right)^{m}\right)$.

First note that $\lambda\left(\cdot ; H_{\lambda}\right)$ is the constant function, $\lambda\left(\cdot ; H_{\lambda}\right)=(2 m(\lambda)+8 m(2 \lambda))^{-1}$. Define the quadratic (in $l$ )

$$
q\left(\mathfrak{d}_{\lambda}, \mathfrak{d}_{2 \lambda}, l\right)=(2 m(\lambda)+8 m(2 \lambda))^{-1}\left(l^{2}+(m(\lambda)+m(2 \lambda)-1) l\right)-\left|\mathfrak{d}_{\lambda}\right|^{2}+\frac{3}{4}\left|\mathfrak{d}_{2 \lambda}\right|^{2} .
$$


Using the formula for $\delta\left(\omega_{\mathfrak{s}}\right)$ derived in Lemma 15, we find, after a small computation, that

$$
f\left(\cdot ; \delta\left(\omega_{\mathfrak{s}}\right)\right)=v \cdot \sum_{n \geq 0} c_{n} q\left(\mathfrak{d}_{\lambda}, \mathfrak{d}_{2 \lambda}, n\right) \lambda^{n-2} .
$$

Now the necessary and sufficient condition that $f\left(\cdot ; \delta\left(\omega_{\mathbf{s}}\right)\right)$ be locally bounded is that the coefficients of $\lambda^{-1}$ and $\lambda^{-2}$ in this expression be zero. Thus $f\left(\cdot ; \delta\left(\omega_{\mathfrak{s}}\right)\right)$ is locally bounded if and only if $c_{1}=c_{0}=0$, or $c_{0}=0$ and $l=1$ is a root of $q\left(\mathfrak{d}_{\lambda}, \mathfrak{d}_{2 \lambda}, l\right)=0$, or $c_{1}=0$ and $\mathfrak{d}_{\lambda}$ is trivial.

Proceeding by induction, assume that $f\left(\cdot ; \delta\left(\omega_{\mathbf{s}}\right)^{l}\right)$ is locally bounded for all $l \leq m$. Then (as we will see) we have

$$
f\left(\cdot ; \delta\left(\omega_{\mathfrak{s}}\right)^{m}\right)=v \cdot \sum_{n \geq 2 m} c_{n} \prod_{l=0}^{m-1} q\left(\mathfrak{d}_{\lambda}, \mathfrak{d}_{2 \lambda}, n-2 l\right) \lambda^{n-2 m} .
$$

Applying $\delta\left(\omega_{\mathfrak{s}}\right)$ once more to $f$ gives

$$
\begin{aligned}
f\left(\cdot ; \delta\left(\omega_{\mathbf{s}}\right)^{m+1}\right)=v & \cdot \sum_{n \geq 2 m+2} c_{n} \prod_{l=0}^{m} q\left(\mathfrak{d}_{\lambda}, \mathfrak{d}_{2 \lambda}, n-2 l\right) \lambda^{n-2 m-2} \\
& +v \cdot c_{2 m+1} \prod_{l=0}^{m} q\left(\mathfrak{d}_{\lambda}, \mathfrak{d}_{2 \lambda}, 2 m+1-2 l\right) \lambda^{-1} \\
& +v \cdot c_{2 m} \prod_{l=0}^{m} q\left(\mathfrak{d}_{\lambda}, \mathfrak{d}_{2 \lambda}, 2 m-2 l\right) \lambda^{-2}
\end{aligned}
$$

Thus again in order for $f\left(\cdot ; \delta\left(\omega_{\mathbf{s}}\right)^{m+1}\right)$ to be locally bounded (assuming $f\left(\cdot ; \delta\left(\omega_{\mathbf{s}}\right)^{l}\right)$ is, for $l \leq m)$ it is necessary and sufficient that the coefficients of $\lambda^{-1}$ and $\lambda^{-2}$ should vanish (which gives formula $(*)$ by induction). Hence, assuming $f\left(\cdot ; \delta\left(\omega_{\mathfrak{s}}\right)^{l}\right)$ is locally bounded for $l \leq m$, we find that $f\left(\cdot ; \delta\left(\omega_{\mathfrak{s}}\right)^{m+1}\right)$ is locally bounded if and only if $c_{2 m+1}=0$ and $c_{2 m}=0$, or

$$
\begin{aligned}
& \text { "odd" } \quad c_{2 m}=0 \text { and } \prod_{l=0}^{m} q\left(\mathfrak{d}_{\lambda}, \mathfrak{d}_{2 \lambda}, 2 l+1\right)=0 \text {, or } \\
& \text { "even" } \quad c_{2 m+1}=0 \text { and } \prod_{l=0}^{m} q\left(\mathfrak{d}_{\lambda}, \mathfrak{d}_{2 \lambda}, 2 l\right)=0 .
\end{aligned}
$$

This inductive calculation shows that if $f$ is a nonzero polynomial function of the form $f=v \cdot \sum_{n \geq k} c_{n} \lambda^{n}$ (with $c_{k} \neq 0$ ), then $f\left(\cdot ; \delta\left(\omega_{s}\right)^{m}\right.$ ) is locally bounded for all $m$ if and only if either $c_{n}=0$ for all even $n$ and the product "odd" vanishes for all $m$ with $2 m+1 \geq k$, or $c_{n}=0$ for all odd $n$ and the product "even" vanishes for all $m$ with $2 m \geq k$. This is equivalent to requiring that $c_{n}=0$ unless the parity of $n$ is the same as the parity of $k$ and $q\left(\mathfrak{d}_{\lambda}, \mathfrak{d}_{2 \lambda}, l\right)=0$ for some $l \leq k$ of the same parity as $k$. Note that the quadratic $q\left(\mathfrak{d}_{\lambda}, \mathfrak{d}_{2 \lambda}, \cdot\right)$ is essentially the same as the quadratic which appears in the statement of the lemma. Thus by step (8), $q\left(\mathfrak{d}_{\lambda}, \mathfrak{d}_{2 \lambda}, \cdot\right)$ has exactly one nonnegative root, which is positive if $\mathfrak{d}_{\lambda}$ is nontrivial. (This justifies the implicit assertion above that at most one of the products "even" or "odd" vanishes for large $n$.) 
Now according to step (7), $v \otimes S\left(\mathfrak{a}_{\mathfrak{s}}\right)$ intersects the image $\beta(\tau)$ in the set of polynomial functions of the form $f=v \cdot \sum_{n \geq k} c_{n} \lambda^{n}$, where if $c_{n} \neq 0$ then the parity of $n$ is the same as that of $v$ and of $k=k\left(\mathfrak{d}_{\lambda}, \mathfrak{d}_{2 \lambda}\right)$. Moreover, by the first sentence of step (1), for such an $f$ in the intersection, $f\left(\cdot ; \delta\left(\omega_{\mathfrak{s}}\right)^{m}\right)$ is locally bounded for all positive integers $m$. Thus $l \leq k$ and the parity of $k$ is the same as that of $l$ (since the intersection is not zero). On the other hand, let $f^{\prime}=v \cdot \sum_{n \geq l} c_{n} \lambda^{n}$ where if $c_{n} \neq 0$ then the parities of $n, l$ and $v$ are all the same, and $l$ is a root of the quadratic $q$. Then $f^{\prime} \in S\left(\mathfrak{a}_{\mathfrak{s}}, \tau\right)$ (since $l>0$ if $\mathfrak{d}_{\lambda}$ is nontrivial by step (8)), and also $f^{\prime}\left(\cdot ; \delta\left(\omega_{s}\right)^{m}\right)$ is locally bounded for all positive integers $m$. Thus $f^{\prime}$ lies in the image of $\beta(\tau)$ (by Lemma 11 and the converse statement in step (1)) and so $l \geq k\left(\mathfrak{d}_{\lambda}, \mathfrak{d}_{2 \lambda}\right)$ (by definition in step (7)).

We have shown that the nonnegative integer $k\left(\mathfrak{d}_{\lambda}, \mathfrak{d}_{2 \lambda}\right)$ is a root of the quadratic equation given in the statement of the present lemma and that

$$
v \otimes S\left(\mathfrak{a}_{\mathfrak{s}}\right) \cap \text { image } \beta(\tau)=\left(v \otimes S\left(\mathfrak{a}_{\mathfrak{s}}\right)\right)^{W_{\mathfrak{s}}} \cap R\left(V_{M} \otimes S\left(\mathfrak{a}_{\mathfrak{s}}\right)\right) .
$$

(10) In order to complete the proof, we must put together the various pieces $v \otimes S\left(\mathfrak{a}_{\mathfrak{s}}\right) \cap$ image $\beta(\tau)$. First note that we have the direct sum decomposition

$$
S(\mathfrak{s}, \tau)=\sum_{\mathfrak{d}_{\lambda} \in \hat{K}} P\left(\mathfrak{d}_{\lambda}\right) \cdot S(\mathfrak{s}, \tau)
$$

(where $P\left(\mathfrak{d}_{\lambda}\right)$ is the projection on the $\mathfrak{d}_{\lambda}$-isotypic component under $\mu$ ). Since restriction clearly respects this direct sum decomposition,

$$
\text { image } \beta(\tau)=\sum_{\mathfrak{d}_{\lambda} \in \hat{K}} P\left(\mathfrak{d}_{\lambda}\right) \cdot \text { image } \beta(\tau) \text {. }
$$

A theorem of Kostant states that the trivial representation of $M$ occurs no more than once in any $\mathfrak{d}_{\lambda} \in \hat{K}$. Thus given $\mathfrak{d}_{\lambda} \in \hat{K}$ such that $P\left(\mathfrak{d}_{\lambda}\right) \cdot V_{M} \neq 0$, there is exactly one $\mathfrak{d}_{2 \lambda} \in \hat{K}_{2 \lambda}$ such that $P\left(\mathfrak{d}_{\lambda}\right) P\left(\mathfrak{d}_{2 \lambda}\right) \mid V_{M} \neq 0$, and then $P\left(\mathfrak{d}_{\lambda}\right) P\left(\mathfrak{d}_{2 \lambda}\right) \mid V_{M}=$ $P\left(\mathfrak{d}_{\lambda}\right) \mid V_{M}$. Also if $v \in V_{M}$ and $P\left(\mathfrak{d}_{\lambda}\right) \cdot v=v$ then already $\mu(w) \cdot v= \pm v$ (where $w$ is the nontrivial element of $W_{\mathbf{s}}$ ). Examining the definition of the endomorphism $R$ given in the statement of the lemma, we see that on $P\left(\mathfrak{d}_{\lambda}\right) \cdot V_{M} \otimes S\left(\mathfrak{a}_{\mathfrak{s}}\right), R$ is just the multiplication operator

$$
P\left(\mathfrak{d}_{\lambda}\right) R=R P\left(\mathfrak{d}_{\lambda}\right)=P\left(\mathfrak{d}_{\lambda}\right) \otimes \lambda^{k}
$$

(where $k=k\left(\mathfrak{d}_{\lambda}, \mathfrak{d}_{2 \lambda}\right)$ and $\mathfrak{d}_{2 \lambda}$ is determined by $\left.\mathfrak{d}_{\lambda}\right)$. Step (9) proves that the Weyl group invariants in the image $R\left(P\left(\mathfrak{d}_{\lambda}\right) \cdot V_{M} \otimes S\left(\mathfrak{a}_{\mathfrak{s}}\right)\right)$ coincides with the space of $f \in P\left(\mathfrak{d}_{\lambda}\right) \cdot S\left(\mathfrak{a}_{\mathfrak{s}}, \tau\right)$ such that $f\left(\cdot ; \delta\left(\omega_{\mathfrak{s}}\right)^{m}\right)$ is locally bounded for all $m$, and by step $(1)$, this space is just $P\left(\mathfrak{d}_{\lambda}\right) \cdot$ image $\beta(\tau)$. Thus the space of $W_{\mathfrak{s}}$-invariants in

$$
R\left(V_{M} \otimes S\left(\mathfrak{a}_{\mathfrak{s}}\right)\right)=\sum P\left(\mathfrak{d}_{\lambda}\right) R\left(V_{M} \otimes S\left(\mathfrak{a}_{\mathfrak{s}}\right)\right)
$$

coincides with the space

$$
\sum P\left(\mathfrak{d}_{\lambda}\right) \cdot \text { image } \beta(\tau)=\text { image } \beta(\tau) .
$$

The proof is finished.

REMARK 17. Lemma 16 is peculiar in that its statement lies in algebraic geometry but the proof seems to require some analysis (at least in step (4)). It would 
seem desirable to have a proof in algebraic geometry, but I do not see how to concoct it. As for Kostant's multiplicity one theorem, I will here give a simple proof along the lines of $[\mathbf{H}$, p. 408, Theorem 4.1].

First note that since $M$ contains the center of $G$, we may assume that $G$ is faithfully embedded in a complexification $G_{c}$. Then $\left(K_{2 \lambda}, M\right)$ and $\left(K, K_{2 \lambda}\right)$ are rank one compact symmetric pairs; the Cartan involutions are $\theta_{2 \lambda}=\operatorname{Ad} a$ and $\theta_{\lambda}=\operatorname{Ad} a^{2}$ respectively, where $a=\exp (\pi \sqrt{-1} H / 2 \lambda(H))$ computed in $G_{c}\left(H \in \mathfrak{a}_{\mathfrak{s}}\right.$, $\lambda(H) \neq 0$ ). If $m(2 \lambda)=0$ we are done (zonal case). If $m(2 \lambda)>1$ then according to another theorem of Kostant [W, Theorem 8.11.3], for each $Y \in \mathfrak{y}_{\lambda}+\mathfrak{y}_{2 \lambda}$ there exists an $m \in M$ such that $\operatorname{Ad} \mathfrak{m} \cdot Y=-Y$. Thus for any $f \in C^{\infty}(K)$ such that $f$ is bi-invariant under $M, f(k)=f\left(k^{-1}\right)$ for all $k \in K$, and again we are done. Finally if $m(2 \lambda)=1$, then a surreptitious peek at the classification shows that $\mathfrak{k}_{2 \lambda}=\mathfrak{m}+$ center of $\mathfrak{k}$, which implies the result.

If $m(2 \lambda)=0,1$ this fact also follows from the general multiplicity one result: if $\pi$ is a principal series representation of $G$ and $\mathfrak{d} \in \hat{K}$ then $[\pi: \mathfrak{d}] \leq 1$. Thus for any irreducible representation $\pi$ of $G$, we have $[\pi: \mathfrak{d}] \leq[\mathfrak{d}: \mathfrak{e}]$ for an appropriate choice $\mathfrak{e} \in \hat{M}$ (by subquotient). But now $K=S O(n)$ and $M=S O(n-1)$, or $K=S U(n) \times$ center and $M=S(U(n-1) \times U(1))$. So we analytically continue $\mathfrak{d}$ to $\tilde{K}=S O(n-1,1)$ or $\tilde{K}=S U(n-1,1)$, and use induction on dimension to obtain $[\mathfrak{d}: \mathfrak{e}] \leq 1$. Thus $[\pi: \mathfrak{d}] \leq 1$.

We are now ready to proceed to the statement of the main result of this paper. Let $G$ be a reductive group of arbitrary rank (satisfying the assumptions at the beginning of $\S 2$ ) and let $H$ be the corresponding Cartan motion group. Let $\Sigma_{+}^{1}$ be the system of positive short (restricted) roots of the pair $\left(\mathfrak{g}, \mathfrak{a}_{\mathfrak{s}}\right)$. For $\lambda \in \Sigma_{+}^{1}$ define

$$
\mathfrak{g}_{\lambda}=\mathfrak{m}+\mathfrak{a}_{\mathfrak{s}}+\mathfrak{n}^{\lambda}+\mathfrak{n}^{2 \lambda}+\mathfrak{n}^{-\lambda}+\mathfrak{n}^{-2 \lambda} .
$$

Then $\mathfrak{g}_{\lambda}$ is a $\theta$-stable reductive subalgebra of $\mathfrak{g}$ which is rank one modulo its center, and $\mathfrak{g}_{\lambda}=\mathfrak{k}_{\lambda}+\mathfrak{s}_{\lambda}=\left(\mathfrak{k} \cap \mathfrak{g}_{\lambda}\right)+\left(\mathfrak{s} \cap \mathfrak{g}_{\lambda}\right)$ is a Cartan decomposition. Let $G_{\lambda}$ be the analytic subgroup of $G$ corresponding to $\mathfrak{g}_{\lambda}$, and set $K_{\lambda}=K \cap G_{\lambda}$ and $M_{\lambda}=$ $M \cap G_{\lambda}$, and let $H_{\lambda}=K_{\lambda} \times \mathfrak{s}_{\lambda}$ be the corresponding Cartan motion group. It is not difficult to extend the result of Lemma 16 to $H_{\lambda}$, but for this it is convenient to introduce the following definition.

DEFINITION. For $\mathfrak{d}_{\lambda} \in \hat{K}_{\lambda}$ define

$$
\left|\mathfrak{d}_{\lambda}\right|_{0}^{2}=B\left(\Lambda_{\lambda}+2 \rho_{\lambda}, \Lambda_{\lambda}\right) / B(\lambda, \lambda)
$$

where $\Lambda_{\lambda}$ is the highest weight of $\mathfrak{d}_{\lambda}$ and $\rho_{\lambda}$ is the half sum of the positive roots of $K_{\lambda}$ with respect to a convenient Cartan subalgebra, and $B$ is the Killing form of $G$. Similarly if $\mathfrak{d}_{2 \lambda} \in \hat{K}_{2 \lambda}\left(K_{2 \lambda}\right.$ corresponding to $\left.\mathfrak{k} \cap\left(\mathfrak{m}+\mathfrak{a}_{\mathfrak{s}}+\mathfrak{n}^{2 \lambda}+\mathfrak{n}^{-2 \lambda}\right)\right)$ set

$$
\left|\mathrm{d}_{2 \lambda}\right|_{0}^{2}=B\left(\Lambda_{2 \lambda}+2 \rho_{2 \lambda}, \Lambda_{2 \lambda}\right) / B(\lambda, \lambda) .
$$

The point is that any compact factor of $G_{\lambda}$ lies in $M_{\lambda}$, so $\left|\mathfrak{d}_{\lambda}\right|_{0}$ and $\left|\mathfrak{d}_{2 \lambda}\right|_{0}$ do not depend on the normalization of $B$ coming from the ambient group $G$ (when $\mathfrak{d}_{\lambda}$ and $\mathfrak{d}_{2 \lambda}$ contain the trivial representation of $\left.M_{\lambda}\right)$. Let $\overline{\mathfrak{s}}_{\lambda}=\left[\mathfrak{g}_{\lambda}, \mathfrak{g}_{\lambda}\right] \cap \mathfrak{s}_{\lambda}$ and $\overline{\mathfrak{a}}_{\lambda}=\overline{\mathfrak{s}}_{\lambda} \cap \mathfrak{a}_{\mathfrak{s}}$ and $\mathfrak{a}^{\lambda}=\operatorname{ker}\left(\lambda: \mathfrak{a}_{\mathfrak{s}} \rightarrow \mathbf{R}\right)$. Then $\mathfrak{a}^{\lambda}$ lies in the center of $H_{\lambda}$ and $\mathfrak{s}_{\lambda}=\mathfrak{a}^{\lambda}+\overline{\mathfrak{s}}_{\lambda}$ and Lemma 16 applies to $K_{\lambda} \times \overline{\mathfrak{s}}_{\lambda}$. Thus if $V, \tau$ is a double unitary representation of $K$ and $\tau_{\lambda}$ is its restriction to $K_{\lambda}$ then

$$
S\left(\mathfrak{s}_{\lambda}, \tau_{\lambda}\right)=S\left(\overline{\mathfrak{s}}_{\lambda}, \tau_{\lambda}\right) \cdot S\left(\mathfrak{a}^{\lambda}\right) .
$$


By Lemma 16 the image of the restriction map $\beta\left(\tau_{\lambda}\right)$ is

$$
I\left(\mathfrak{a}_{\mathfrak{s}}, \tau_{\lambda}, \lambda\right)=\text { image } \beta\left(\tau_{\lambda}\right)=\sum P\left(\mathfrak{d}_{\lambda}\right) V_{M_{\lambda}} \otimes S\left(\mathfrak{a}^{\lambda}\right) \lambda^{k} \mathbf{C}\left[\lambda^{2}\right]
$$

where $k=k\left(\mathfrak{d}_{\lambda}, \mathfrak{d}_{2 \lambda}\right)$ is the nonnegative solution of

$$
k^{2}+(m(\lambda)+m(2 \lambda)-1) k=\left|\mathfrak{d}_{\lambda}\right|_{0}^{2}-\frac{3}{4}\left|\mathfrak{d}_{2 \lambda}\right|_{0}^{2} .
$$

(Note that if $B_{\lambda}$ is the Killing form of $G_{\lambda}$ then $B_{\lambda}(\lambda, \lambda)^{-1}=2 m(\lambda)+8 m(2 \lambda)$.) Now we can describe the image of $\beta(\tau)$.

DEFINITION. Define $I\left(\mathfrak{a}_{\mathfrak{s}}, \tau\right) \subseteq V \otimes S\left(\mathfrak{a}_{\mathfrak{s}}\right)$ by

$$
I\left(\mathfrak{a}_{\mathfrak{s}}, \tau\right)=\bigcap\left\{I\left(\mathfrak{a}_{\mathfrak{s}}, \tau_{\lambda}, \lambda\right) \mid \lambda \in \Sigma_{+}^{1}\right\}
$$

where $I\left(\mathfrak{a}_{\mathfrak{s}}, \tau_{\lambda}, \lambda\right)$ is described in the preceding paragraph.

THEOREM 19. Let $f: \mathfrak{a}_{\mathfrak{s}} \rightarrow V$. Then the following are equivalent:

(a) $f$ lies in the image of the restriction map $\beta(\tau): S(\mathfrak{s}, \tau) \rightarrow V \otimes S\left(\mathfrak{a}_{\mathfrak{s}}\right)$.

(b) $f \in I\left(\mathfrak{a}_{\mathfrak{s}}, \tau\right)$.

(c) $f \in S\left(\mathfrak{a}_{\mathfrak{s}}, \tau\right)$ and $f\left(\cdot ; \delta\left(\omega_{\mathfrak{s}}\right)^{m}\right)$ is locally bounded for all positive integers $m$.

(d) $f(\operatorname{Ad} m \cdot h)=\tau_{1}(m) f(h) \tau_{2}\left(m^{-1}\right)$ for all $m \in M^{*}$ (= the normalizer of $\mathfrak{a}_{\mathfrak{s}}$ in $K)$ and $f \in \bigcap\left\{I\left(\mathfrak{a}_{\mathfrak{s}}, \tau_{\lambda}, \lambda\right) \mid \lambda \in \Sigma_{+}^{1}, \lambda\right.$ simple $\}$.

PrOOF. (a) implies (c) follows from Lemma 11 and 15 and the converse comes from the first five steps of Lemma 16 (together with the last part of Lemma 11). To show (a) implies (d), let $\tilde{f} \in S(\mathfrak{s}, \tau)$ be such that $\tilde{f} \mid \mathfrak{a}_{\mathfrak{s}}=f$, and let $H_{\lambda} \subseteq H$ be the Cartan motion group described above (used to define $I\left(\mathfrak{a}_{\mathfrak{s}}, \tau_{\lambda}, \lambda\right)$ ). Assume $\lambda$ is a simple root. The $M^{*}$-invariance in (d) follows from the fact that $f \in S\left(\mathfrak{a}_{\mathfrak{s}}, \tau\right)$ (the second condition in the definition). That $f \in I\left(\mathfrak{a}_{\mathfrak{s}}, \tau_{\lambda}, \lambda\right)$ can be seen by noting that $\tilde{f} \mid \mathfrak{s}_{\lambda} \in S\left(\mathfrak{s}_{\lambda}, \tau_{\lambda}\right)$ and $f$ is simply the restriction of $\tilde{f} \mid \mathfrak{s}_{\lambda}$ to $\mathfrak{a}_{\mathfrak{s}}$.

To show that (d) implies (b), recall that every short root is conjugate under $W_{s}$ to a simple root. It is easy to see that

$$
\operatorname{Ad} w \cdot I\left(\mathfrak{a}_{\mathfrak{s}}, \tau_{\lambda}, \lambda\right)=I\left(\mathfrak{a}_{\mathfrak{s}}, \tau_{w \cdot \lambda}, w \cdot \lambda\right) .
$$

Since in $(\mathrm{d})$ we assume that $f \in I\left(\mathfrak{a}_{\mathfrak{s}}, \tau_{\lambda}, \lambda\right)$ for $\lambda$ simple, we have $f=w \cdot f \in$ $\bigcap I\left(\mathfrak{a}_{\mathfrak{s}}, \tau_{\lambda}, \lambda\right)$, intersection over all short roots. So now it only remains to show that (b) implies (a).

(1) The first job is to show that $I\left(\mathfrak{a}_{\mathfrak{s}}, \tau\right) \subseteq S\left(\mathfrak{a}_{\mathfrak{s}}, \tau\right)$. For this we will show that $M$ is generated by $\bigcup\left\{M_{\lambda} \mid \lambda \in \Sigma_{+}^{1}\right\}$. So let $M^{1}$ be the subgroup of $M$ generated by $\cup M_{\lambda}$. Of course $M^{1}$ contains the identity component of $M$. Next let us show that $K \cap$ center of $G$ lies in $M^{1}$.

Let $\mathfrak{g}_{c}=\mathfrak{a}_{c}+\sum \mathfrak{g}_{c}^{\alpha}(\alpha \in \Phi)$ be a root space decomposition, where the Cartan subalgebra $\mathfrak{a}_{c} \supseteq \mathfrak{a}_{\mathfrak{s}}$ and $\Phi$ is the set of roots $\mathfrak{g}_{c}, \mathfrak{a}_{c}$. Let $\Gamma \subseteq \Phi$ be a maximal strongly orthogonal system of real roots [W1, pp. 93-97]. "Real" means that we can find $X_{\alpha} \in \mathfrak{g}_{c}^{\alpha} \cap \mathfrak{g}$ and $X_{-\alpha} \in \mathfrak{g}_{c}^{-\alpha} \cap \mathfrak{g}$ such that $X_{\alpha}, X_{-\alpha}$ and $H_{\alpha}=\left[X_{\alpha}, X_{-\alpha}\right] \in \mathfrak{a}_{\mathfrak{s}}$ form a Chevalley basis (over $\mathbf{R}$ ) for a subalgebra $\mathfrak{s l}(2, \alpha) \subseteq \mathfrak{g}$ which is isomorphic to $\mathfrak{s l}(2, \mathbf{R})$. "Strongly orthogonal" implies that the elements of $\mathfrak{s l}(2, \alpha)$ commute with those of $\mathfrak{s l}(2, \beta)$ for $\alpha \neq \beta(\alpha, \beta \in \Gamma)$. Let $\mathfrak{s l}(2, \Gamma)$ be the subalgebra of $\mathfrak{g}$ spanned by $\mathfrak{a}_{\mathfrak{s}}$ and the $\mathfrak{s l}(2, \alpha)$ for $\alpha \in \Gamma$. Let $S l(2, \Gamma)$ be the analytic subgroup of $G$ generated by $\mathfrak{s l}(2, \Gamma)$. "Maximal" implies that $\mathfrak{t}=\mathfrak{k} \cap \mathfrak{s l}(2, \Gamma)$ is a Cartan subalgebra of $\mathfrak{k}$ and $T=K \cap S l(2, \Gamma)$ is a Cartan subgroup of $K$. 
Now $K \cap$ center of $G$ lies in $T$ and clearly it also lies in the center of $\operatorname{Sl}(2, \Gamma)$. On the other hand, since $\mathfrak{a}_{\mathfrak{s}} \subseteq \mathfrak{s l}(2, \Gamma), K \cap$ center of $S l(2, \Gamma)$ lies in $M$. But $K \cap$ center of $\operatorname{Sl}(2, \Gamma)$ is generated by $\exp \left(\mathfrak{a}_{c} \cap \mathfrak{k}\right.$ ) (contained in the identity component of $M)$ and the various centers of $\mathfrak{s l}(2, \alpha)(\alpha \in \Gamma)$, and these are contained in $M_{\lambda}$ for $\lambda=\alpha \mid \mathfrak{a}_{\mathfrak{s}}$ (or $\lambda=\frac{1}{2} \alpha \mid \mathfrak{a}_{\mathfrak{s}}$ ). Thus $M^{1}$ contains $K \cap$ center of $G$.

So now we may as well assume that $G$ is a semisimple adjoint group. Let $\tilde{G}$ be a covering group of $G$ which is contained in a simply connected complexification $\tilde{G}_{c}$, and let $\tilde{M}$ be the centralizer of $\mathfrak{a}_{\mathfrak{s}}$ in $\tilde{K}$. According to [W1, pp. 28, 213], $\tilde{M}$ is generated by its identity component and the $\tilde{m}_{\lambda}=\exp \left(\pi \sqrt{-1} H_{\lambda}\right)$, where $\exp$ is computed in $\tilde{G}_{c}$ and $H_{\lambda} \in \mathfrak{a}_{\mathfrak{s}}$ satisfies $B\left(h, H_{\lambda}\right)=c \lambda(h)$ (for all $h \in \mathfrak{a}_{\mathfrak{s}}$ ) and $\lambda\left(H_{\lambda}\right)=2$. Now the covering map $\tilde{G} \rightarrow G$ takes $\tilde{M}$ onto $M$, so $M$ is generated by its identity component and the images $m_{\lambda}$ of the $\tilde{m}_{\lambda}$. On the other hand, applying the same construction and lemmas in [W1] to $G_{\lambda}$, we see that $\tilde{m}_{\lambda}$ can be computed relative to $\tilde{G}_{\lambda}$, and $m_{\lambda} \in G_{\lambda}$. So we see that $M$ is generated by $\bigcup M_{\lambda}, \lambda \in \Sigma_{+}^{1}$.

(2) If $f \in I\left(\mathfrak{a}_{\mathfrak{s}}, \tau\right)$ then $f \in I\left(\mathfrak{a}_{\mathfrak{s}}, \tau_{\lambda}, \lambda\right)$ for each $\lambda$. Thus $f(h)=\tau_{1}(m) f(h) \tau_{2}\left(m^{-1}\right)$ for all $h \in \mathfrak{a}_{\mathrm{s}}$ and $m \in M_{\lambda}$, and since this holds for any $\lambda \in \Sigma_{+}^{1}$, we find that $f(h)$ is $M$-invariant. Now we can ask whether $f(w \cdot h)=\mu(w) \cdot f(h)$ for $w \in W_{\mathfrak{s}}$. But since $f \in I\left(\mathfrak{a}_{\mathfrak{s}}, \tau_{\lambda}, \lambda\right)$ this holds for the simple reflections, hence for all $W_{\mathfrak{s}}$. Thus $f$ satisfies the second condition in the definition of $S\left(\mathfrak{a}_{\mathfrak{s}}, \tau\right)$. (Note that this gives (b) implies (d).)

(3) Now we verify that each $f \in I\left(\mathfrak{a}_{\mathfrak{s}}, \tau\right)$ satisfies the first condition in the definition of $S\left(\mathfrak{a}_{\mathfrak{s}}, \tau\right)$. Such an $f$ lies in $I\left(\mathfrak{a}_{\mathfrak{s}}, \tau_{\lambda}, \lambda\right)=$ image $\beta\left(\tau_{\lambda}\right)$ for all $\lambda$ (Lemma 16), so if $h \in \mathfrak{a}_{\mathfrak{s}}$ and $k \in K_{\lambda}$ and $\operatorname{Ad} k \cdot h=h$ then $f(h)=\mu(k) \cdot f(h)$. Note that this equation holds for all $k \in K_{\lambda}$ if $h \in \mathfrak{a}^{\lambda}$, and if $h \notin \mathfrak{a}^{\lambda}$ then $\operatorname{Ad} k \cdot h=h$ implies $k \in M_{\lambda}$.

Let $\Lambda: \mathfrak{a}_{\mathfrak{s}} \rightarrow \mathbf{C}$ be real linear and let $K_{\Lambda}=\{k \in K \mid \operatorname{Ad} k \cdot \Lambda=\Lambda\}$ (this has nothing to do with the notation $K_{\lambda}$ ). In the reductive group $G$ associated to $H$, there is a standard construction of a parabolic subgroup $P_{\Lambda}$ of $G$ corresponding to $\Lambda$. Namely $P_{\Lambda}=L_{\Lambda} N$ where $L_{\Lambda}$ is the centralizer of $\Lambda$ in $G$ and $N$ is from the Iwasawa decomposition. It is easy to see that $K_{\Lambda}=K \cap L_{\Lambda}=K \cap P_{\Lambda}$. On the other hand, if $K_{\Lambda}^{\circ}$ and $L_{\Lambda}^{\circ}$ denote the identity components, then $L_{\Lambda}=L_{\Lambda}^{\circ} \cdot M$ [W1, p. 74] so $K_{\Lambda}=K_{\Lambda}^{\circ} \cdot M$. Define $H_{\Lambda} \in \mathfrak{a}_{\mathfrak{s c}}$ by $B\left(h, H_{\Lambda}\right)=\Lambda(h)$ for all $h \in \mathfrak{a}_{\mathfrak{s}}$. Then $\mathfrak{k}_{\Lambda}=\mathfrak{m}+\sum\left\{\mathfrak{k}_{\lambda} \mid \lambda\left(H_{\Lambda}\right)=0\right\}$ is the Lie algebra of $K_{\Lambda}$, so $K_{\Lambda}$ is generated by $M$ and the $K_{\lambda}$ such that $\lambda\left(H_{\Lambda}\right)=0$ (i.e., the $\left.K_{\lambda} \subseteq K_{\Lambda}\right)$. Hence to show that $\mu(k) \cdot f\left(H_{\Lambda}\right)=f\left(H_{\Lambda}\right)$ for all $k \in K_{\Lambda}$, it suffices to show this for $k \in M$ (which was done in step (2)) and for $k \in K_{\lambda}$ when $\lambda\left(H_{\Lambda}\right)=0$ (which we noted above).

(4) Now we complexify everything as in the last part of the proof of Lemma 11. If $f \in I\left(\mathfrak{a}_{\mathfrak{s}}, \tau\right)$ then $f \in S\left(\mathfrak{a}_{\mathfrak{s}}, \tau\right)$ so $f$ extends to a rational function $\tilde{f}: \mathfrak{s}_{c}^{\prime} \rightarrow V$ defined everywhere on $\mathfrak{s}_{c}^{\prime}$. If we show that $\tilde{f}$ is actually a polynomial function defined on $\mathfrak{s}_{c}$ we will be done.

Define $\sigma_{\lambda}$ and $\sigma$ in $S\left(\mathfrak{a}_{\mathfrak{s} c}\right)$ by

$$
\sigma_{\lambda}=\prod\left\{\mu^{2} \mid \mu \in \Sigma_{+}^{1} \text { and } \mu \neq \lambda\right\} \quad \text { and } \sigma=\sum\left\{\sigma_{\lambda} \mid \lambda \in \Sigma_{+}^{1}\right\} .
$$

It is clear that $\sigma$ is $W_{\mathfrak{s}}$-invariant, so it extends to a polynomial function $\tilde{\sigma}$ on $\mathfrak{s}_{c}$ which is $\operatorname{Ad} K_{c}$-invariant. Let $\mathfrak{s}_{c}^{\prime \prime}$ be the set of non-zeros of $\tilde{\sigma}$. Also $\sigma_{\lambda}$ is invariant by $W_{\lambda}$ (the Weyl group of $\left.\left(\mathfrak{g}_{\lambda}, \mathfrak{a}_{\mathfrak{s}}\right)\right)$ and so extends to an $\operatorname{Ad} K_{\lambda c}$-invariant polynomial $\tilde{\sigma}_{\lambda}$ on $\mathfrak{s}_{\lambda c}$. Let $\mathfrak{s}_{\lambda c}^{\prime \prime}$ be the set of $x \in \mathfrak{s}_{\lambda c}$ such that $\tilde{\sigma}(x) \neq 0$ and $\tilde{\sigma}_{\lambda}(x) \neq 0$. 
If $x \in \mathfrak{s}_{c}^{\prime \prime}$ write $x=x_{s}+x_{n}$ where $x_{s}$ is semisimple and $x_{n}$ is nilpotent and $\left[x_{s}, x_{n}\right]=0$ (this relative to the Lie algebra structure of $\mathfrak{g}_{c}$ ). By [K\&R, p. 772], after conjugating by $K_{c}$, we may assume that $x_{s} \in \mathfrak{a}_{\mathfrak{s c}}$ and then $\tilde{\sigma}(x)=\sigma\left(x_{s}\right) \neq 0$. From the form of $\sigma$, either $x_{s}$ is regular or exactly one short root vanishes on $x_{s}$. If $x_{s}$ is regular then $x=x_{s} \in \mathfrak{a}_{\mathfrak{s} c}^{\prime}$ and $\sigma(x) \neq 0$ and $\sigma_{\lambda}(x) \neq 0$ so $x \in \mathfrak{s}_{\lambda c}^{\prime \prime}$ for every $\lambda \in \Sigma_{+}^{1}$. If exactly one root, say $\lambda$, vanishes on $x_{s}$ then $x_{n} \in \mathfrak{s}_{\lambda c}\left(\mathfrak{g}_{\lambda}\right.$ is the centralizer of $x_{s}$ in $\left.\mathfrak{g}\right)$ and $\tilde{\sigma}_{\lambda}\left(x_{n}\right)=0$ [K\&R, p. 772] and $\tilde{\sigma}_{\lambda}(x)=\sigma_{\lambda}\left(x_{s}\right)=\sigma\left(x_{s}\right) \neq 0$ so $x \in \mathfrak{s}_{\lambda c}^{\prime \prime}$ for this $\lambda \in \Sigma_{+}^{1}$. Thus each point of $\mathfrak{s}_{c}^{\prime \prime}$ is conjugate under $K_{c}$ to an element of $\mathfrak{s}_{\lambda c}^{\prime \prime}$ for some $\lambda$.

We have to do a bit better. Let $C$ be a compact subset of $\mathfrak{s}_{c}^{\prime \prime}$. Then I claim that there are compact sets $C_{\lambda} \subseteq \mathfrak{s}_{\lambda c}^{\prime \prime}$ such that every point of $C$ is conjugate under $K_{c}$ to a point of $C_{\lambda}$ for some $\lambda$ (depending on the point of $C$ ). For $\varepsilon>0$ define

$$
U_{\lambda \varepsilon}=\left\{x \in \mathfrak{s}_{\lambda c}:|\tilde{\sigma}(x)|>\varepsilon,\left|\tilde{\sigma}_{\lambda}(x)\right|>\varepsilon \text { and }|x|<1 / \varepsilon\right\} .
$$

Then the $U_{\lambda \varepsilon}$ have compact closure in $\mathfrak{s}_{\lambda c}^{\prime \prime}$ and form a nested open covering of $\mathfrak{s}_{\lambda c}^{\prime \prime}$ (as $\varepsilon \rightarrow 0$ ). Moreover, the orbit map $(k, x) \mapsto \operatorname{Ad} k \cdot x: K_{c} \times U_{\lambda \varepsilon} \rightarrow \mathfrak{s}_{c}^{\prime \prime}$ is a submersion. (The Jacobian determinant of ad $x: \mathfrak{k}_{c} \cap \mathfrak{g}_{\lambda c}^{\perp} \rightarrow \mathfrak{s}_{c} \cap \mathfrak{g}_{\lambda c}^{\perp}$ divides an appropriate power of $\sigma_{\lambda}(x) \neq 0$, where $\mathfrak{g}_{\lambda c}^{\perp}$ is the perpendicular complement of $\mathfrak{g}_{\lambda c}$ in $\mathfrak{g}_{c}$.) Thus the $\operatorname{Ad} K_{c} \cdot U_{\lambda \varepsilon}$ form an open covering of $\mathfrak{s}_{c}^{\prime \prime}$ as $\lambda$ and $\varepsilon$ vary. Choosing $\varepsilon>0$ such that $C \subseteq \bigcup_{\lambda} \operatorname{Ad} K_{c} \cdot U_{\lambda \varepsilon}$, we can take $C_{\lambda}$ to be the closure of $U_{\lambda \varepsilon}$.

Let $f \in I\left(\mathfrak{a}_{\mathfrak{s}}, \tau\right)$ and let $\tilde{f}: \mathfrak{s}_{c}^{\prime} \rightarrow V$ be its extension to a rational function. But also, by definition, $f \in I\left(\mathfrak{a}_{\mathfrak{s} c}, \tau_{\lambda}, \lambda\right)$, so $f$ extends to a polynomial function $f_{\lambda}: \mathfrak{s}_{\lambda c} \rightarrow V$ by Lemma 16 , and $f_{\lambda}=\tilde{f}$ on $\mathfrak{s}_{\lambda c} \cap \mathfrak{s}_{c}^{\prime}$. If $C$ is any compact set in $\mathfrak{s}_{c}^{\prime \prime}$ and $C_{\lambda}$ are as above, then

$$
\sup _{C \cap \mathfrak{s}_{c}^{\prime}}|\tilde{f}| \leq \max _{\lambda} \sup _{C_{\lambda} \cap \mathfrak{s}_{c}^{\prime}}\left|f_{\lambda}\right|<\infty .
$$

Thus $\tilde{f}$ is locally bounded on $\mathfrak{s}_{c}^{\prime \prime}$, and the complement of $\mathfrak{s}_{c}^{\prime}$ in $\mathfrak{s}_{c}^{\prime \prime}$ is a thin set (the zeros of $\tilde{\pi}^{2}$ ) so $\tilde{f}$ extends to a rational function defined on all of $\mathfrak{s}_{c}^{\prime} \cup \mathfrak{s}_{c}^{\prime \prime}$ [G\&R, p. 19].

(5) In order to complete the proof, all that remains is to show that the complement of $\mathfrak{s}_{c}^{\prime} \cup \mathfrak{s}_{c}^{\prime \prime}$ in $\mathfrak{s}_{c}$, that is the algebraic set of common zeros of $\tilde{\pi}^{2}$ and $\tilde{\sigma}$, has codimension two in $\mathfrak{s}_{c}$ [G\&R, p. 247]. For this it suffices to show that $\tilde{\pi}^{2}$ and $\tilde{\sigma}$ are relatively prime in $S\left(\mathfrak{s}_{c}\right)$. Let $p$ be the greatest common divisor of $\tilde{\pi}^{2}$ and $\tilde{\sigma}$. Since $\tilde{\pi}^{2}$ and $\tilde{\sigma}$ are Ad $K_{c}$-invariant, for any $k \in K_{c}, \operatorname{Ad} k \cdot p$ is also a g.c.d. for $\tilde{\pi}^{2}$ and $\tilde{\sigma}$. Thus $\operatorname{Ad} k \cdot p=c(k) p$ where $c$ is a character of $K_{c}$. Hence $p \mid \mathfrak{a}_{\mathfrak{s} c}$ is a common factor of $\pi^{2}=\tilde{\pi}^{2} \mid \mathfrak{a}_{\mathfrak{s} c}$ and $\sigma=\tilde{\sigma} \mid \mathfrak{a}_{\mathfrak{s} c}$ of the same degree as $p$. But from their definitions it is clear that $\pi^{2}$ and $\sigma$ are relatively prime, so $p \mid \mathfrak{a}_{\mathfrak{s} c}$ is constant, and $\tilde{\pi}^{2}$ and $\tilde{\pi}$ are relatively prime.

This completes the proof of the theorem.

In fact it can be shown that the g.c.d. of any two elements of $I(\mathfrak{s})$ is actually Ad $K_{c}$-invariant (i.e. $c(k)=1$ in the preceding paragraph).

Unfortunately, Theorem 19 is difficult to use for two reasons. First, the numbers $k\left(\mathfrak{d}_{\lambda}, \mathfrak{d}_{2 \lambda}\right)$ and the projections $P\left(\mathfrak{d}_{\lambda}\right)$ are hard to calculate. Also this theorem expresses the image of $\beta(\tau)$ as an intersection which is again hard to compute (partly because the $P\left(\mathfrak{d}_{\lambda}\right)$ do not commute as $\lambda$ varies). This is especially bad since we will be interested in the image of this intersection under certain maps. In this case, an approximate answer is more useful than the exact solution. 
Let $m$ be the largest of the numbers $5\left|\Lambda_{1}+\Lambda_{2}\right|$ as $\Lambda_{1}$ and $\Lambda_{2}$ vary over the highest weights of the representations $k \mapsto \tau_{1}(k)$ and $k \mapsto \tau_{2}\left(k^{-1}\right)$, respectively, of $K$ on $V$, and

$$
\left|\Lambda_{1}+\Lambda_{2}\right|^{2}=B\left(\Lambda_{1}+\Lambda_{2}+2 \rho_{k}, \Lambda_{1}+\Lambda_{2}\right)
$$

(where $\rho_{k}$ is the half sum of the positive roots of $\mathfrak{k}$ ). Let $P$ be a semistandard parabolic subgroup of the reductive group $G$ associated to $H$ [W1, p. 69]. Set $K_{P}=K \cap P$ and put

$$
C\left(K_{P}\right)=\left\{v \in V \mid \tau_{1}(k) \cdot v \cdot \tau_{2}\left(k^{-1}\right)=v \text { for all } k \in K_{P}\right\} .
$$

Let $\mathfrak{u}$ be the Lie algebra of the unipotent radical of $P$ and let $\pi(P) \in S\left(\mathfrak{a}_{\mathfrak{s}}\right)$ be the polynomial function $h \mapsto \operatorname{det}(\operatorname{ad} h \mid \mathfrak{u})$. Set $\pi(P)=1$ if $P=G$.

DEFINITION. Put

$$
J^{\prime}\left(\mathfrak{a}_{\mathfrak{s}}, \tau\right)=\sum_{P} C\left(K_{P}\right) \otimes S\left(\mathfrak{a}_{\mathfrak{s}}\right) \pi(P)^{m}
$$

where the vector space sum is over all semistandard parabolics of $G$. Then $J^{\prime}\left(\mathfrak{a}_{\mathfrak{s}}, \tau\right)$ is stable under the action of $W_{\mathfrak{s}}$. Let $J\left(\mathfrak{a}_{\mathfrak{s}}, \tau\right)$ be the space of $W_{\mathfrak{s}}$-invariants in $J^{\prime}\left(\mathfrak{a}_{\mathfrak{s}}, \tau\right)$.

COROLLARY 20. Image $\beta(\tau)=I\left(\mathfrak{a}_{\mathfrak{s}}, \tau\right)$ contains $J\left(\mathfrak{a}_{\mathfrak{s}}, \tau\right)$. Moreover in the case

$$
V=\mathscr{L}\left(E_{\mathfrak{\jmath}}\right) \quad \text { and } \quad \tau_{1}(k) v \tau_{2}(l)=\mu_{\mathfrak{\vartheta}}(k) \circ v \circ \mu_{\mathfrak{\jmath}}(l),
$$

$J\left(\mathfrak{a}_{\mathfrak{s}}, \tau\right)$ is a subalgebra of the image of $\beta_{\mathfrak{0}}: \mathfrak{X} \rightarrow \mathscr{L}\left(E_{\mathfrak{0}}\right) \otimes S\left(\mathfrak{a}_{\mathfrak{s}}\right)$.

PROOF. Because of Theorem 19(d), it suffices to show that

$$
C\left(K_{P}\right) \otimes S\left(\mathfrak{a}_{\mathfrak{s}}\right) \pi(P)^{m} \subseteq \bigcap\left\{\sum_{\mathfrak{d}_{\lambda}} P\left(\mathfrak{d}_{\lambda}\right) \cdot V_{M} \otimes S\left(\mathfrak{a}_{\mathfrak{s}}\right) \lambda^{k} \mid \lambda \text { is simple }\right\}
$$

$\left(k=k\left(\mathfrak{d}_{\lambda}, \mathfrak{d}_{2 \lambda}\right)\right)$ for each semistandard $P \subseteq G$, and this amounts to verifying that $\lambda^{k}$ divides $\pi(P)^{m}$ when $P\left(\mathfrak{d}_{\lambda}\right) \cdot V_{M} \cap C\left(K_{P}\right) \neq\{0\}$.

Let $\mathfrak{l}+\mathfrak{u}$ be the Levi decomposition of the Lie algebra of $P$, and let $\Sigma_{P}=$ $\Sigma_{\mathfrak{l}}+\Sigma_{\mathfrak{u}} \subseteq \Sigma$ be the set of roots for the adjoint action of $\mathfrak{a}_{\mathfrak{s}}$ on $\mathfrak{l}+\mathfrak{u}$ [W1, pp. 14, 72]. Then

$$
\pi(P)=\prod\left\{\lambda^{m(\lambda)}(2 \lambda)^{m(2 \lambda)} \mid \lambda \in \Sigma_{\mathfrak{u}}\right\}
$$

and $\pi(P)^{5}$ is divisible by

$$
\lambda^{2 m(\lambda)+8 m(2 \lambda)} \quad\left(\lambda \in \Sigma_{\mathfrak{u}}\right)
$$

(since $m(2 \lambda) \leq m(\lambda)-1$ ).

If $\lambda \in \Sigma_{1} \cap \Sigma_{+}^{1}$ then $K_{\lambda} \subseteq K_{P}$ and $P\left(\mathfrak{d}_{\lambda}\right) \cdot V_{M} \cap C\left(K_{P}\right) \neq\{0\}$ implies that $\mathfrak{d}_{\lambda}$ is the trivial representation of $K_{\lambda}$. Thus $k=k\left(\mathfrak{d}_{\lambda}, \mathfrak{d}_{2 \lambda}\right)=0$ and $\lambda^{k}$ divides $\pi(P)^{m}$.

So suppose $\lambda \in \pm \Sigma_{\mathfrak{u}} \cap \Sigma_{+}^{1}$. From equation (18) it is clear that $k \leq\left|\mathfrak{d}_{\lambda}\right|_{0}$ (since $\left.\left|\mathrm{d}_{2 \lambda}\right|_{0}^{2} \geq 0\right)$. Now

$$
\begin{aligned}
\left|\mathfrak{d}_{\lambda}\right|_{0}^{2} & =(2 m(\lambda)+8 m(2 \lambda)) B_{\lambda}\left(\Lambda_{\lambda}+2 \rho_{\lambda}, \Lambda_{\lambda}\right) \\
& \leq(2 m(\lambda)+8 m(2 \lambda)) B\left(\Lambda_{\lambda}+2 \rho_{\lambda}, \Lambda_{\lambda}\right) .
\end{aligned}
$$

(The last inequality follows from the fact that $B_{\lambda}$ is computed via a trace on the subspace $\mathfrak{g}_{\lambda} \subseteq \mathfrak{g}$ ). Now let $\omega_{\lambda}$ be the Casimir operator $K_{\lambda}$ computed relative 
to $B \mid \mathfrak{k}_{\lambda} \times \mathfrak{k}_{\lambda}$ and let $\omega_{k}$ be the Casimir operator of $K$ relative to $B$. Then for $v \in P\left(\mathfrak{d}_{\lambda}\right) \cdot V_{M}$,

$$
\mu\left(\omega_{\lambda}\right) \cdot v=B\left(\Lambda_{\lambda}+2 \rho_{\lambda}, \Lambda_{\lambda}\right) \cdot v
$$

and

$$
\mu\left(\omega_{k}\right)\left|V_{M}=\sum \mu\left(\omega_{\lambda}\right)\right| V_{M} \quad\left(\lambda \in \Sigma_{+}^{1}\right) .
$$

Since this is a sum of positive operators, the largest eigenvalue of any of the $\mu\left(\omega_{\lambda}\right) \mid V_{M}$ does not exceed $m / 5$, the largest eigenvalue of $\mu\left(\omega_{k}\right)$. Thus $k\left(\mathfrak{d}_{\lambda}, \mathfrak{d}_{2 \lambda}\right) \leq$ $(2 m(\lambda)+8 m(2 \lambda)) m / 5$ and $\lambda^{k}$ divides $\pi(P)^{m}$ (where $\lambda \in \pm \Sigma_{\mathfrak{u}}$ ).

In order to prove the last statement it suffices to show that $J\left(\mathfrak{a}_{\mathfrak{5}}, \tau\right)$ is a subalgebra of $\mathscr{L}\left(E_{\mathfrak{0}}\right) \otimes S\left(\mathfrak{a}_{\mathrm{s}}\right)$. But this follows from the observation that if $P$ and $Q$ are standard parabolic subgroups of $G$, then $C\left(K_{P}\right) \cdot C\left(K_{Q}\right)=C\left(K_{P \cap Q}\right)$ and $\pi(P \cap Q)$ is the least common multiple of $\pi(P)$ and $\pi(Q)$.

4. Applications to harmonic analysis. We now return to the problem of parameterizing $\left[I_{c 0}(H)\right]$; the solution was initiated in Lemmas 13 and 14 . We showed there that any spherical function of type $\mathfrak{d}$ was equivalent to an irreducible factor in the Jordan-Hölder series of certain induced spherical functions. To complete the parameterization we will show that in fact these induced spherical functions are irreducible. First we need to expand the notation a bit.

Let $H=K \times \mathfrak{s}$ be a Cartan motion group and let $\mathfrak{s}=\mathfrak{a}_{\mathfrak{s}}+\mathfrak{z}$ be the orthogonal decomposition described at the beginning of $\S 2$. If $x \in H$, write uniquely $x=$ $(\kappa(x), H(x)+z)$ where $\kappa(x) \in K, H(x) \in \mathfrak{a}_{\mathfrak{s}}$ and $z \in \mathfrak{z}$. If $\Lambda: \mathfrak{a}_{\mathfrak{s}} \rightarrow \mathbf{C}$ is reallinear, then $x \mapsto \Lambda \circ H(x)$ gives a linear extension to $\mathfrak{s}$ with $\mathfrak{z}$ contained in the kernel. As in Lemma 13 , let $K_{\Lambda}$ be the centralizer of $\Lambda \circ H$, and let $\mathfrak{e} \in \hat{K}_{\Lambda}$. Let $\pi=\pi(\mathfrak{e}, \Lambda)$ be the representation of $H$ induced from $(k, X) \mapsto \mu_{\mathfrak{e}}(k) e^{\Lambda H(X)}: K_{\Lambda} \times$ $\mathfrak{s} \rightarrow \mathscr{L}\left(E_{\mathfrak{e}}\right)$. Formula (9) gives a formula for the corresponding induced spherical function $\Phi(\mathfrak{e}, \Lambda, \mathfrak{d})$.

LEMMA 21. Let $\Phi$ be a spherical function of type $\mathfrak{d} \in \hat{K}$. Then there exists a real-linear function $\Lambda: \mathfrak{a}_{\mathfrak{s}} \rightarrow \mathbf{C}$ and an $\mathfrak{e} \in \hat{K}_{\Lambda}$ with $[\mathfrak{d}: \mathfrak{e}]>0$ such that $\Phi$ is equivalent to the spherical function $\Phi(\mathfrak{e}, \Lambda, \mathfrak{d})$ realized on

$$
F_{\mathfrak{0} \mid \mathrm{e}}=\operatorname{Hom}_{K_{\Lambda}}\left(E_{\mathfrak{0}}, E_{\mathrm{e}}\right)
$$

by the formula

$$
\Phi(\mathfrak{e}, \Lambda, \mathfrak{o}, x) \cdot l=\int_{K} e^{\Lambda H(x k)} l \circ \mu_{\mathfrak{o}}\left(\kappa\left(k^{-1} x k\right)\right) d k .
$$

On the other hand the $\Phi(\mathfrak{e}, \Lambda, \mathfrak{d})$ are spherical functions of type $\mathfrak{d}$ (that is the corresponding representation of $I_{c \mathcal{D}}(H)$ is irreducible). Finally $\Phi\left(\mathfrak{e}_{1}, \Lambda_{1}, \mathfrak{d}\right)$ is equivalent to $\Phi\left(\mathfrak{e}_{2}, \Lambda_{2}, \mathfrak{d}\right)$ if and only if there is a $w \in W_{\mathfrak{s}}$ such that $\Lambda_{1}=w \cdot \Lambda_{2}$ and $\mathfrak{e}_{1}=w \cdot \mathfrak{e}_{2}$.

Proof. To show irreducibility of $\Phi(\mathfrak{e}, \Lambda, \mathfrak{d})$, it suffices to show that the corresponding representation of $\mathfrak{X}$ is irreducible (because $H$ is connected so $\mathfrak{X}$ is dense in the space of $K$-central compactly supported distributions in the weak topology induced by real-analytic functions, such as $\Phi(\mathfrak{e}, \Lambda, \mathfrak{d}))$. Recalling the formula

$$
\Phi\left(\mathfrak{e}, \Lambda, \mathfrak{o}, 1 ; D_{K}\right) \cdot l=l \circ\left(\mu_{\mathfrak{o}} \otimes e^{\Lambda}\right)\left(\beta_{\mathfrak{o}}\left(D_{K}\right)\right)
$$

$\left(D_{K} \in \mathfrak{X}\right.$ and $\left.l \in F_{\mathfrak{0} \mid \mathfrak{e}}\right)$ for this representation of $\mathfrak{X}$, we see that it will suffice to show that $D_{K} \mapsto\left(1 \otimes e^{\Lambda}\right) \circ \beta_{\mathfrak{0}}\left(D_{K}\right)$ takes $\mathfrak{X}$ onto the centralizer of $\mu_{\mathfrak{\jmath}}\left(K_{\Lambda}\right)$ in $\mathscr{L}\left(E_{\mathfrak{0}}\right)$. 
More generally, we show that if $\tau=\left(\tau_{1}, \tau_{2}\right): K \times K \rightarrow \mathscr{L}(V)$ is a double representation of $K$ and $\Lambda \in \mathfrak{a}_{\mathfrak{s} c}$, then

$$
\left(1 \otimes e^{\Lambda}\right) \circ \beta(\tau, S(\mathfrak{s}, \tau))=C\left(K_{\Lambda}\right)=\left\{v \in V \mid \tau_{1}(k) v \tau_{2}\left(k^{-1}\right)=v \text { for all } k \in K_{\Lambda}\right\} .
$$

Recall then in the proof of Theorem 19, step (3), it was observed that $K_{\Lambda}=$ $K \cap P_{\Lambda}$ where $P_{\Lambda}=L_{\Lambda} N$ is a semistandard parabolic subgroup of $G$ and $L_{\Lambda}$ is the centralizer of $\Lambda$ in $G$. Now the roots of the unipotent radical of $P_{\Lambda}$ are precisely the roots which are positive on $\Lambda$, so, in the notation around Corollary $20, e^{\Lambda}\left(\pi\left(P_{\Lambda}\right)^{m}\right) \neq 0$. Choose $D \in S\left(\mathfrak{a}_{\mathfrak{s}}\right)$ such that

$$
e^{\Lambda}\left(D \pi\left(P_{\Lambda}\right)^{m}\right)=1 \quad \text { and } \quad e^{w \cdot \Lambda}(D)=0 \quad \text { if } w \cdot \Lambda \neq \Lambda
$$

$\left(w \in W_{\mathfrak{s}}\right)$. Then by Corollary 20 , if $L \in C\left(K_{\Lambda}\right)$ and $\left[W_{\Lambda}\right]$ is the order of the centralizer of $\Lambda$ in $W_{\mathfrak{s}}$, we have that

$$
\left[W_{\Lambda}\right]^{-1} \sum_{w \in W_{s}} \tau_{1}\left(w^{-1}\right) L \tau_{2}(w) \otimes \operatorname{Ad} w^{-1}\left(D \pi\left(P_{\Lambda}\right)^{m}\right)
$$

lies in the image $\beta(\tau, S(\mathfrak{s}, \tau))$ and is mapped to $L$ by $1 \otimes e^{\Lambda}$. This proves the irreduciblity.

As for the equivalences, Lemma 13 tells us that $\Phi\left(\mathfrak{e}_{1}, \Lambda_{1}, \mathfrak{d}\right)$ and $\Phi\left(\mathfrak{e}_{2}, \Lambda_{2}, \mathfrak{d}\right)$ are disjoint unless $\Lambda_{1}=w \cdot \Lambda_{2}$ for some $w \in W_{\mathbf{s}}$. If $m \in M^{*}$ represents $w$ then $K_{w \Lambda_{2}}=m K_{\Lambda_{2}} m^{-1}$ and $w \cdot \mathfrak{e}_{2}$ is the equivalence class of $k \mapsto \mu_{\mathfrak{e}_{2}}\left(m^{-1} \mathrm{~km}\right)$. Clearly $l \mapsto l \circ \mu_{\mathfrak{o}}\left(m^{-1}\right): F_{\mathfrak{d} \mid \mathfrak{e}_{2}} \rightarrow F_{\mathfrak{d} \mid w \cdot \mathfrak{e}_{2}}$ defines an equivalence of $\Phi\left(\mathfrak{e}_{2}, \Lambda_{2}, \mathfrak{d}\right)$ and $\Phi\left(w \cdot \mathfrak{e}_{2}, w \cdot \Lambda_{2}, \mathfrak{d}\right)$. Thus we may as well assume $\Lambda_{1}=\Lambda_{2}$, so assuming $\Phi\left(\mathfrak{e}_{1}, \Lambda, \mathfrak{d}\right)$ is equivalent to $\Phi\left(\mathfrak{e}_{2}, \Lambda, \mathfrak{d}\right)$ we are to show $\mathfrak{e}_{1}=\mathfrak{e}_{2}$. But this follows from

$$
\mu_{\mathfrak{0}}\left(\alpha_{\mathfrak{e}_{1}}\right) \in C\left(K_{\Lambda}\right)=\left(1 \otimes e^{\Lambda}\right) \circ \beta_{\mathfrak{o}}(\mathfrak{X}) .
$$

Now if $\Phi: G \rightarrow \mathscr{L}(F)$ is any spherical function, then Lemma 13 says that there exists $\Lambda$ and $\mathfrak{e} \in \hat{K}_{\Lambda}$ and an isomorphism $Q: F \rightarrow F_{\mathfrak{o} \mid e}$ which gives an equivalence of the representations of $\mathfrak{X}$ deduced from $\Phi$ and $\Phi(\mathfrak{e}, \Lambda, \mathfrak{d})$. But then by Proposition 7 , the real-analytic function on $H$ (connected) $x \mapsto \Phi(\mathfrak{e}, \Lambda, \mathfrak{d}, x)-Q \Phi(x) Q^{-1}$ has zero Taylor series at $1 \in H$, so $[\Phi]=[\Phi(\mathfrak{e}, \Lambda, \mathfrak{d})]$.

We are now able to parameterize the nonunitary dual $[H]$ of $H$ (up to Naimark equivalence). The result is already suggested by Lemma 14 .

LEMMA 22. Let $\Lambda: \mathfrak{a}_{\mathfrak{s}} \rightarrow \mathbf{C}$ be real-linear and let $\mathfrak{e} \in \hat{K}_{\Lambda}$. Let $\pi(\mathfrak{e}, \Lambda)$ be the representation of $H$ induced from $(k, X) \mapsto \mu_{\mathrm{e}}(k) e^{\Lambda H(X)}: K_{\Lambda} \times \mathfrak{s} \rightarrow \mathscr{L}\left(E_{\mathrm{e}}\right)$. Then $\pi(\mathfrak{e}, \Lambda)$ is TCI. Also $\pi\left(\mathfrak{e}_{1}, \Lambda_{1}\right)$ and $\pi\left(\mathfrak{e}_{2}, \Lambda_{2}\right)$ are Naimark equivalent if and only if $\Lambda_{1}=w \cdot \Lambda_{2}$ and $\mathfrak{e}_{1}=w \cdot \mathfrak{e}_{2}$ for some $w \in W_{\mathfrak{s}}$. Finally any TCI representation of $H$ is Naimark equivalent to $\pi(\mathfrak{e}, \Lambda)$ for some $\mathfrak{e}, \Lambda$.

PROOF. If we could show that $\pi(\mathfrak{e}, \Lambda)$ is TCI, then the statement about equivalence would follow from Corollary 3 and Lemma 21, and the final assertion would be a consequence of Lemma 14. To see that $\pi=\pi(\mathfrak{e}, \Lambda)$ is TCI, note first that $\pi\left(C_{c}(G)\right)$ is a $K \times K$-submodule of $\mathscr{L}(E)$ (where $E$ is the representation space of $\pi$ ). If $\pi$ were not TCI, that is $\pi\left(C_{c}(G)\right)$ were not dense in $\mathscr{L}(E)$ (simple convergence), then there would exist a double $K$-module $V$ and a nonzero double $K$-homomorphism $T: \mathscr{L}(E) \rightarrow V$ such that $T(\pi(f))=0$ for all $f \in C_{c}(G)$. Thus to prove $\pi$ is TCI, it suffices to show that given a unitary double $K$-module $V, \tau$ and a double 
$K$-homomorphism $T: \mathscr{L}(E) \rightarrow V$, if $T(\pi(x))=0$ for all $x \in H$ then $T=0$ (see [H-CIII, p. 130]).

But $T(\pi(x))$ is given by an Eisenstein integral of Harish-Chandra. Using the notation of Remark 8, formula (10) simplifies substantially for Cartan motion groups. For $x=(k, X)$ write $\kappa(x)=k$ and $p(x)=X$. Set $v=\psi_{T}(1) \in V$ (where $1 \in \mathscr{L}\left(E_{\sigma}\right)$ is the unity), so $v$ is an arbitrary vector in $V$ satisfying

$$
\tau_{1}(m) v=v \tau_{2}(m) \quad\left(m \in K_{\Lambda}\right) \quad \text { and } \quad \tau_{1}\left(\alpha_{\mathfrak{e}}\right) \cdot v=v .
$$

Define $\mu(k) \cdot v=\tau_{1}(k) v \tau_{2}\left(k^{-1}\right)$. Then the Eisenstein integral is given by

$$
E\left(P, \mathfrak{e}, \Lambda, \psi_{T}, x\right)=\tau(\kappa(x)) \int_{K} \mu(k) \cdot v e^{\Lambda H(x k)} d k
$$

(where $P=K_{\Lambda} \times \mathfrak{s}$ and $v=\psi_{T}(1)$ ).

Now $E\left(P, \mathfrak{e}, \Lambda, \psi_{T}\right)$ defines a linear functional on $S(\mathfrak{s}, \tau)$ given by

$$
\begin{aligned}
\left\langle E\left(P, \mathfrak{e}, \Lambda, \psi_{T}\right) \mid \sum v_{n} \otimes D_{s n}\right\rangle & =\sum\left(E\left(P, \mathfrak{e}, \Lambda, \psi_{T}, 1 ; D_{s n}\right) \mid v_{n}\right) \\
& =\sum\left(v \mid v_{n}\right) e^{\Lambda \circ H}\left(1 ; D_{s n}\right) \\
& =\left(v \mid\left(1 \otimes e^{\Lambda}\right) \circ \beta\left(\tau, \sum v_{n} \otimes D_{s n}\right)\right)
\end{aligned}
$$

(where $(\cdot \cdot)$ is the inner product on $V$ ). Thus to show that $T \neq 0$ implies $T(\pi(x)) \not \equiv$ 0 (and so that $\pi=\pi(\mathfrak{e}, \Lambda)$ is TCI), it suffices to show that $\left(v \mid\left(1 \otimes e^{\Lambda}\right) \circ \beta(\tau, D)\right) \neq 0$ for some $D \in S(\mathfrak{s}, \tau)$ (for any given $v \neq 0$ in $C\left(K_{\Lambda}\right)$ ). But the existence of such a $D$ follows from

$$
\left(1 \otimes e^{\Lambda}\right) \circ \beta(\tau, S(\mathfrak{s}, \tau))=C\left(K_{\Lambda}\right)
$$

which was shown in the proof of Lemma 21 .

Next we describe the Fell topology on $[H]$. For $\lambda, \nu \in \mathfrak{a}_{s}^{\vee}$ define

$$
|\lambda+\sqrt{-1} \nu|^{2}=B(\lambda, \lambda)+B(\nu, \nu) \text {. }
$$

Let $\mathscr{F}_{c}$ be the set of all pairs $(\mathfrak{e}, \Lambda)$ where $\Lambda: \mathfrak{a}_{\mathfrak{s}} \rightarrow \mathbf{C}$ is real-linear and $\mathfrak{e} \in \hat{K}_{\Lambda}$. Given $(\mathfrak{e}, \Lambda) \in \mathscr{F}_{c}$, if $\varepsilon>0$ is sufficiently small then $\left|\Lambda-\Lambda^{\prime}\right|<\varepsilon$ implies $K_{\Lambda^{\prime}} \subseteq K_{\Lambda}$. Then

$$
U=\left\{\left(\mathfrak{e}^{\prime}, \Lambda^{\prime}\right) \in \mathscr{F}_{c}:\left|\Lambda-\Lambda^{\prime}\right|<\varepsilon \text { and }\left[\mathfrak{e} \mid K_{\Lambda^{\prime}}: \mathfrak{e}^{\prime}\right]>0\right\}
$$

defines a basis for the neighborhoods of $(\mathfrak{e}, \Lambda)$ in the topology we give $\mathscr{F}_{c}$. If $\mathfrak{d} \in \hat{K}$, let $\mathscr{F}_{c}(\mathfrak{d})=\left\{(\mathfrak{e}, \Lambda) \in \mathscr{F}_{c} \mid[\mathfrak{d}: \mathfrak{e}]>0\right\}$, so $\mathscr{F}_{c}(\mathfrak{d})$ is open in $\mathscr{F}_{c}$. Now $W_{\mathfrak{s}}$ acts on $\mathscr{F}_{c}$ and $\mathscr{F}_{c}(\mathfrak{d})$ by $w \cdot(\mathfrak{e}, \Lambda)=(w \cdot \mathfrak{e}, w \cdot \Lambda)$. Let $\mathscr{F}_{c} / W_{\mathfrak{s}}$ and $\mathscr{F}_{c}(\mathfrak{d}) / W_{\mathfrak{s}}$ be the quotient spaces by this action of $W_{s}$, with the quotient topologies.

THEOREM 24. The map $(\mathfrak{e}, \Lambda) \mapsto[\pi(\mathfrak{e}, \Lambda)]: \mathscr{F}_{c} / W_{\mathfrak{s}} \rightarrow[H]$ defines a homeomorphism of $\mathscr{F}_{c} / W_{\mathbf{s}}$ with the above topology onto the Banach dual $[H]$ of $H$ with the Fell topology. Similarly $(\mathfrak{e}, \Lambda) \mapsto[\Phi(\mathfrak{e}, \Lambda, \mathfrak{d})]: \mathscr{F}_{c}(\mathfrak{d}) / W_{\mathfrak{s}} \rightarrow\left[I_{c \mathfrak{d}}(H)\right]$ is a homeomorphism.

PROOF. Lemmas 21 and 22 say that the maps in the statement of the theorem are one to one and onto. Also by Proposition 5, it suffices to show that the second map is a homeomorphism (since $\mathscr{F}_{c}(\mathfrak{d})$ is open in $\mathscr{F}_{c}$ ). 
Let $\left(\mathfrak{e}_{\alpha}, \Lambda_{\alpha}\right)$ be a net converging to $(\mathfrak{e}, \Lambda)$ in $\mathscr{F}_{c}(\mathfrak{d}) / W_{\mathfrak{s}}$, so we may as well assume that $\Lambda_{\alpha}$ converges to $\Lambda$. First suppose that $K_{\Lambda_{\alpha}}$ and $\mathfrak{e}_{\alpha}=\mathfrak{e}^{\prime}$ are constant, independent of $\alpha$. Then the formula in Lemma 21 implies

$$
\begin{aligned}
\lim \operatorname{tr} & \Phi\left(\mathfrak{e}^{\prime}, \Lambda_{\alpha}, \mathfrak{d}, x\right) \\
& =\lim d\left(\mathfrak{e}^{\prime}\right)^{-1} \int_{K} \operatorname{tr}\left\{P\left(\mathfrak{e}^{\prime}\right) \mu_{\mathfrak{d}}\left(\kappa\left(k^{-1} x k\right)\right)\right\} e^{\Lambda_{\alpha} H(x k)} d k \\
& =\sum\left\{\left[\mathfrak{e}^{\prime \prime}: \mathfrak{e}^{\prime}\right] \operatorname{tr} \Phi\left(\mathfrak{e}^{\prime \prime}, \Lambda, \mathfrak{d}, x\right) \mid \mathfrak{e}^{\prime \prime} \in \hat{K}_{\Lambda} \text { and }\left[\mathfrak{d}: \mathfrak{e}^{\prime \prime}\right]>0\right\}
\end{aligned}
$$

uniformly on compacta. Using the Chinese remainder theorem, choose $f_{\mathfrak{e}} \in I_{c \mathfrak{d}}(H)$ such that $\Phi\left(\mathfrak{e}^{\prime \prime}, \Lambda, \mathfrak{d}, f_{\mathfrak{e}}\right)$ is the identity matrix if $\mathfrak{e}^{\prime \prime}=\mathfrak{e}$ and is zero otherwise. Then

$$
\lambda_{\alpha}(f)=\left[\mathfrak{e}: \mathfrak{e}^{\prime}\right]^{-1} \operatorname{tr} \Phi\left(\mathfrak{e}^{\prime}, \Lambda_{\alpha}, \mathfrak{d}, f * f_{\mathfrak{e}}\right)
$$

converges to $\operatorname{tr} \Phi(\mathfrak{e}, \Lambda, \mathfrak{d}, f)$ for all $f \in I_{c \mathfrak{d}}(H)$. Now in general there are only a finite number of choices for $K_{\Lambda \alpha}$ and $\mathfrak{e}_{\alpha}$, so considering in turn each of the cofinal subnets on which these are constant, we find that the map $\mathscr{F}_{c}(\mathfrak{d}) / W_{\mathfrak{s}} \rightarrow\left[I_{c \mathfrak{o}}(H)\right]$ is continuous.

Let $\left(\mathfrak{e}_{\alpha}, \Lambda_{\alpha}\right)$ be a net in $\mathscr{F}_{c}(d) / W_{\mathfrak{s}}$, set $\Phi_{\alpha}=\Phi\left(\mathfrak{e}_{\alpha}, \Lambda_{\alpha}, \mathfrak{d}\right)$ and suppose that $\left[\Phi_{\alpha}\right]$ converges to $[\Phi]=[\Phi(\mathfrak{e}, \Lambda, \mathfrak{d})]$ in $\left[I_{c \mathfrak{d}}(H)\right]$. Recall that this means given any $\lambda \in$ $\mathscr{A}(\Phi)$ we can choose $\lambda_{\alpha}^{\prime} \in \mathscr{A}\left(\Phi_{\alpha}\right)$ such that $\lim \lambda_{\alpha}^{\prime}(f)=\lambda(f)$ for all $f \in I_{c \mathfrak{d}}(H)$. Fix $f$ such that $\Phi(f)=1$ and set $\lambda_{\alpha}(D)=\lambda_{\alpha}^{\prime}(f * D)$ (where $D \in \mathfrak{X}$ is thought of as a distribution with support at $\{1\})$. Then $\lim \lambda_{\alpha}(D)=\lambda(D)$ for all $D \in \mathfrak{X}$. This is to say that the identity map from $\left[I_{c \mathfrak{d}}(H)\right]$ onto $\left[\beta_{\mathfrak{d}}(\mathfrak{X})\right]$ is continuous. So now it is sufficient to show that the obvious map $\left[\beta_{\mathfrak{d}}(\mathfrak{X})\right] \rightarrow \mathscr{F}_{c}(\mathfrak{d}) / W_{\mathfrak{s}}$ is continuous.

Now if $D \in 1 \otimes I\left(\mathfrak{a}_{\mathfrak{s}}\right)$, a subalgebra of the center of $\beta_{\mathfrak{d}}(\mathfrak{X})$, then $D$ is represented by scalars, so if $\lambda(1) \neq 0$ we have

$$
\lim e^{\Lambda_{\alpha}}(D)=\lim \lambda_{\alpha}(D) / \lambda_{\alpha}(1)=e^{\Lambda}(D) .
$$

Thus we may as well assume that $\Lambda_{\alpha}$ converges to $\Lambda$ and, as above, we may suppose $\mathfrak{e}_{\alpha}=\mathfrak{e}^{\prime}$ is independent of $\alpha$. But then

$$
\lim \operatorname{tr} \Phi\left(\mathfrak{e}^{\prime}, \Lambda_{\alpha}, \mathfrak{d}, 1 ; D\right)=\sum\left\{\left[\mathfrak{e}, \mathfrak{e}^{\prime}\right] \operatorname{tr} \Phi(\mathfrak{e}, \Lambda, \mathfrak{d}, 1 ; D) \mid \mathfrak{e} \in \hat{K}_{\Lambda} \text { and }[\mathfrak{d}: \mathfrak{e}]>0\right\}
$$

for all $D \in \mathfrak{X}$. Now the rest of the proof, the continuity of $\left[\beta_{\mathfrak{d}}(\mathfrak{X})\right] \rightarrow \mathscr{F}_{c}(\mathfrak{d}) / W_{\mathfrak{s}}$, is a consequence of the following lemma.

LEMMA 25. Let $A$ be an associative algebra with 1 over $\mathbf{C}$ and suppose every TCI representation of $A$ is finite dimensional. Let $\left[\Phi_{\alpha}\right]$ be a net in $[A]$ such that $\lim \operatorname{tr} \Phi_{\alpha}(a)$ exists for all $a \in A$. Then

$$
\lim \operatorname{tr} \Phi_{\alpha}(a)=\sum m(\Phi) \operatorname{tr} \Phi(a)
$$

(finite sum) where $m(\Phi) \neq 0$ if and only if $\left[\Phi_{\alpha}\right]$ converges to $[\Phi]$ in the Fell topology on $[A]$.

Proof. Define the linear map $t: A \rightarrow \mathbf{C}$ by $t(a)=\lim \operatorname{tr} \Phi_{\alpha}(a)$. Set $d=$ $\lim \operatorname{tr} \Phi_{\alpha}(1)$ and put

$$
I=\{b \in A \mid t(a b)=0 \text { for all } a \in A\} .
$$

Then $t(a b)=t(b a)$ (for $a, b \in A$ ) and $I$ is a two sided ideal. 
(1) $\operatorname{dim}(A / I) \leq d^{2}$. To see this, suppose that $b_{1}, \ldots, b_{n}$ have linearly independent images in $A / I$. If $V=\mathrm{C}^{n}$ then we have a linear map $\lambda: A \rightarrow V^{\vee}$ defined by

$$
\langle\lambda(a) \mid c\rangle=t\left(\left(\sum c_{i} b_{i}\right) a\right) \quad\left(c=\left(c_{1}, \ldots, c_{n}\right) \in \mathbf{C}^{n}\right)
$$

and $c \neq 0$ implies $\langle\lambda(a) \mid c\rangle \neq 0$ for some $a \in A$ (by definition of $I$ ). Thus $\lambda$ is surjective, so we can choose $a_{1}, \ldots, a_{n} \in A$ such that $t\left(b_{i} a_{j}\right)=\delta_{i j}$. (In particular the bilinear form $(a, b) \mapsto t(a b)$ is nondegenerate on $A / I$.) But then the linear maps $b \mapsto \operatorname{tr} \Phi_{\alpha}\left(b a_{j}\right)$ (where $\left.j=1, \ldots, n\right)$ are linearly independent for $\alpha$ sufficiently large. Thus

$$
n \leq \operatorname{dim} \Phi_{\alpha}(A)=d^{2} \quad(\alpha \text { large }) .
$$

(2) Let $\Phi_{1}, \ldots, \Phi_{k}$ be the various inequivalent irreducible representations of $A$ which factor through $A / I$. Then $a \mapsto \operatorname{tr} \Phi_{i}(a)$ defines a linear functional on $A / I$, so by step (1) we may choose $a_{i} \in A$ such that

$$
\operatorname{tr} \Phi_{i}(a)=t\left(a a_{i}\right)=\lim \operatorname{tr} \Phi_{\alpha}\left(a a_{i}\right) .
$$

Thus $\left[\Phi_{\alpha}\right]$ converges to $\left[\Phi_{i}\right]$ for each $i$.

(3) Now we show that if $\left[\Phi_{\alpha}\right]$ converges to $[\Phi]$ in $[A]$ then $\Phi$ factors through $A / I$. If $B \in \mathscr{L}\left(\mathbf{C}^{d}\right)$, let

$$
z^{d}+\sum_{k=1}^{d} \varphi_{k}(B) z^{d-k}
$$

be its characteristic polynomial. Then there exist universal polynomials $p_{k}$ such that

$$
\varphi_{k}(B)=p_{k}\left(\operatorname{tr}(B), \ldots, \operatorname{tr}\left(B^{k}\right)\right) .
$$

(Upper triangulate $B$ and apply [Weyl, p. 39].) In particular

$$
\phi_{k}(a)=\lim \varphi_{k}\left(\Phi_{\alpha}(a)\right)=\lim p_{k}\left(\operatorname{tr} \Phi_{\alpha}(a), \ldots, \operatorname{tr} \Phi_{\alpha}\left(a^{k}\right)\right)=p_{k}\left(t(a), \ldots, t\left(a^{k}\right)\right)
$$

exists. Now a matrix satisfies its own characteristic polynomial, so

$$
\Phi_{\alpha}\left(a^{d}\right)+\sum_{k=1}^{d} \varphi_{k}\left(\Phi_{\alpha}(a)\right) \Phi_{\alpha}\left(a^{d-k}\right)=0
$$

for all large $\alpha$. If $\lambda \in \mathscr{A}(\Phi)$ choose $\lambda_{\alpha} \in \mathscr{A}\left(\Phi_{\alpha}\right)$ such that $\lim \lambda_{\alpha}(a)=\lambda(a)$ for all $a \in A$. Then

$$
\begin{aligned}
0 & =\lim \lambda_{\alpha}\left(a^{d}+\sum \varphi_{k}\left(\Phi_{\alpha}(a)\right) a^{d-k}\right) \\
& =\lim \left\{\lambda_{\alpha}\left(a^{d}\right)+\sum \varphi_{k}\left(\Phi_{\alpha}(a)\right) \lambda_{\alpha}\left(a^{d-k}\right)\right\} \\
& =\lambda\left(a^{d}\right)+\sum \phi_{k}(a) \lambda\left(a^{d-k}\right) \\
& =\lambda\left(a^{d}+\sum \phi_{k}(a) a^{d-k}\right) .
\end{aligned}
$$

Since this holds for all $\lambda \in \mathscr{A}(\Phi)$ we see that $\Phi(a)$ satisfies the polynomial equation

$$
\Phi(a)^{d}+\sum \phi_{k}(a) \Phi(a)^{d-k}=0
$$

for all $a \in A$. 
Now if $a \in I$ then $\phi_{k}(a b)=p_{k}\left(t(a b), \ldots, t\left((a b)^{k}\right)\right)=p_{k}(0, \ldots, 0)=0$ for all $b \in A$. Then we have $\Phi(a b)^{d}=0$ for all $b \in A$, which implies $\Phi(a)=0$ since $\Phi$ is irreducible. That is if $\left[\Phi_{\alpha}\right]$ converges to $[\Phi]$ then $\Phi$ factors through $A / I$.

(4) Next we show that the ring $A / I$ is semisimple. Suppose, if possible, that $b \neq 0$ lies in the nilpotent radical of $A / I$. Replacing $b$ by $a b^{n}$ for suitable $a \in A$ and $n \in \mathbf{N}$, we can suppose that $t(b)=1$ and $t\left(b^{l}\right)=0$ for $l>1$. Now the universal polynomials mentioned in the preceding paragraph are determined by solving the recursion relation

$$
(k+1) \phi_{k+1}(a)+\sum_{m=0}^{k} \phi_{m}(a) t\left(a^{k-m+1}\right)=0
$$

(where $\phi_{0}(a)=1$ ). Substituting $t(b)=1$ and $t\left(b^{l}\right)=0$ for $l>0$, we obtain $\phi_{k}(b)=(-1)^{k} / k$ !. As in the previous paragraph, this gives

$$
0=\lambda\left(b^{d}+\sum_{k=1}^{d} \frac{(-1)^{k} b^{d-k}}{k !}\right)
$$

for any linear functional $\lambda: A / I \rightarrow \mathrm{C}$. In particular, if $l(b)$ denotes left multiplication by $b$ on $A / I$, then $l(b)$ satisfies the polynomial equation

$$
\sum_{k=0}^{d} \frac{(-1)^{k} z^{d-k}}{k !}=0 .
$$

But this is not divisible by the minimal polynomial of $l(b)$ (which is of the form $z^{m}=0$ since $l(b)$ is nilpotent). This contradiction shows that $A / I$ is semisimple.

(5) So now write $1=e_{1}+\cdots+e_{k}$ as a sum of orthogonal central idempotents, so $a \mapsto t\left(a e_{i}\right)$ is a central linear functional on the simple ideal $e_{i} \cdot A / I$. If $\Phi_{1}, \ldots, \Phi_{k}$ are as in step (2), then we can suppose the enumeration is such that $\Phi_{i}\left(e_{j}\right)=\delta_{i j} \cdot 1$. Then $t\left(a e_{i}\right)=m_{i} \operatorname{tr} \Phi_{i}(a)$ with $m_{i}=t\left(e_{i}\right) / \operatorname{tr} \Phi_{i}\left(e_{i}\right) \neq 0$ (because otherwise $t\left(a e_{i}\right)=$ 0 for all $a \in A$ so $\left.e_{i} \in I\right)$. So now for any $a \in A$

$$
t(a)=t\left(a \sum e_{i}\right)=\sum m_{i} \operatorname{tr} \Phi_{i}(a),
$$

and, taking account of steps (2) and (3), the result is proved.

It is curious to observe that the parameterization we have obtained for the Naimark dual of $H$ is the same as one would get if Mackey's theory of induced representations were applicable to these nonunitary representations. (It is not.) Let

$$
\mathscr{F}=\left\{(\mathfrak{e}, \Lambda) \in \mathscr{F}_{c} \mid \Lambda=\sqrt{-1} \lambda \text { where } \lambda \text { is real valued }\right\} .
$$

Then Mackey's theory says that the unitary dual $\hat{H}$ of $H$ is parameterized by $\mathscr{F} / W_{\mathbf{s}}$ exactly as in Theorem 24 .

Next we turn to the Plancherel formula for $H$. It is shown in [W1, p. 333] that if $\pi$ is an admissible representation of $H$ on a Hilbert space and if $f \in C_{c}^{\infty}(H)$ then $\pi(f)$ is a trace class operator and

$$
f \mapsto \Theta_{\pi}(f)=\operatorname{tr} \pi(f) \quad\left(f \in C_{c}^{\infty}(H)\right)
$$


is a central distribution depending only on the Naimark equivalence class of $\pi$. In fact for $r \geq 0$ let

$$
\mathscr{C}_{r}(H)=\left\{f \in C^{\infty}(H): \sup _{H}\left|f\left(D_{1} ;(k, X) ; D_{2}\right)\right| p(X) e^{r|X|}<\infty\right\}
$$

where $D_{1}, D_{2} \in \mathfrak{H}$, and $p$ is a polynomial function on $\mathfrak{s}$ and $|X|^{2}=B(X, X)$. If $\pi=\pi(\mathfrak{e}, \Lambda)$ and $|\operatorname{Re} \Lambda|^{2}=B(\operatorname{Re} \Lambda, \operatorname{Re} \Lambda) \leq r^{2}$, then clearly $f \mapsto \pi(f)$ is a trace class operator for $f \in \mathscr{C}_{r}(H)$, and $f \mapsto \operatorname{tr} \pi(f)$ is continuous with respect to the obvious seminorms on $\mathscr{C}_{r}(H)$.

A standard computation, [W1, p. 464], gives a formula for the characters $\Theta_{\pi}$ of the irreducible (induced) representations $\pi=\pi(\mathfrak{e}, \Lambda)$ we have parameterized. Namely

$$
\Theta_{\pi}(f)=\int_{K_{\Lambda} \times \mathfrak{s}} \int_{K} f\left(k m k^{-1}, \operatorname{Ad} k \cdot X\right) \operatorname{tr} \mu_{\mathrm{e}}(m) e^{\Lambda(X)} d k d m d X
$$

for $f \in \mathscr{C}_{r}(H)$. Note that $\Theta_{\pi}$ is given by a measure, but not generally by a locally integrable class function (because the support of $\Theta_{\pi}$ is $\left\{k m k^{-1} \mid k \in K\right.$ and $\left.m \in K_{\Lambda}\right\} \times \mathfrak{s}$ which usually has empty interior). In particular these formulas hold for $\mathscr{C}_{0}(H)$ (the Schwartz space) and unitary $\pi$ (the real part of $\Lambda, \Re \Lambda=0$ ).

Normalize the Lebesgue measures on subspaces of $\mathfrak{s}$ and $\mathfrak{s}^{\vee}$ so that the measure of the unit cube with respect to $B$ is one, and normalizc the Haar measures of closed subgroups of $K$ to have total mass one. Choose the Haar measure $d x=d k d X$ on $H$. Let $\Sigma_{+}$be the set of positive roots of $\left(\mathfrak{g}, \mathfrak{a}_{\mathfrak{s}}\right)$ and $m(\lambda)$ be the multiplicity of $\lambda \in \Sigma_{+}$, with $m\left(\frac{1}{2} \lambda\right)=0$ if $\frac{1}{2} \lambda$ is not in $\Sigma_{+}$. Let $\rho_{\mathbf{s}}=\frac{1}{2} \sum\left\{m(\lambda) \lambda \mid \lambda \in \Sigma_{+}\right\}$and set $\rho_{\lambda}=\left(\rho_{\mathfrak{s}}, \lambda\right) /(\lambda, \lambda)$. Define the constant

$$
\gamma\left(P_{0}\right)=\prod_{\lambda} \Gamma\left(\frac{1}{4} m\left(\frac{1}{2} \lambda\right)+\rho_{\lambda}\right)(2 \pi /(\lambda, \lambda))^{m(\lambda) / 2} / \Gamma\left(\frac{1}{2} m(\lambda)+\frac{1}{4} m\left(\frac{1}{2} \lambda\right)+\rho_{\lambda}\right)
$$

where the product is over all $\lambda \in \Sigma_{+}$and $\Gamma$ is the classical gamma function.

THEOREM 28. With the above normalization of Haar measures, the Plancherel formula for $H$ is given by

$$
\begin{aligned}
f(1)=\left[W_{\mathfrak{s}}\right]^{-1}(\sqrt{2} \pi)^{-\operatorname{dim} s}(\sqrt{2})^{-\operatorname{dim} \mathfrak{a}_{\mathfrak{s}}} \gamma\left(P_{0}\right) & \\
& \times \int_{\mathfrak{a}_{\mathfrak{s}}} \prod_{\lambda \in \Sigma_{+}}|(\lambda, \nu)|^{m(\lambda)} \sum_{\mathfrak{e} \in \hat{M}} d(\mathfrak{e}) \Theta_{\pi(\mathfrak{e}, i \nu)}(f) d \nu
\end{aligned}
$$

for all $f \in \mathscr{C}_{0}(H)$ (absolute convergence).

PROOF. The proof of this theorem appears in [K\& L] except for the computation of the normalizing constant. However, since the proof is so simple, I will reproduce it. First, substituting formula (26) and applying the Plancherel formula for $M$, we have

$$
\sum_{\mathfrak{e} \in \hat{M}} d(\mathfrak{e}) \Theta_{\pi(\mathfrak{e}, i \nu)}(f)=\int_{K} \int_{\mathfrak{s}} f(1, X) e^{i<\operatorname{Ad} k \cdot \nu|X\rangle} d X d k .
$$

It turns out that

$$
\gamma\left(P_{0}\right)=\int_{N^{-}} e^{-2 \rho_{0} H(n)} d n=(\sqrt{2})^{-\operatorname{dim}(K / M)} \operatorname{vol}(K / M) .
$$


(Here, Haar measures on closed subgroups of $G$ and on $K / M$ are normalized to be the Riemannian measures corresponding to the invariant Riemann metric induced by $B$ as in [H-CI, p. 106].) Substituting these formulas into the integral given in the statement of the theorem and using $\left[\mathbf{H}\right.$, p. 381] and $\operatorname{dim}(K / M)=\operatorname{dim} \mathfrak{s}-\operatorname{dim} \mathfrak{a}_{\mathfrak{s}}$ gives

$$
\begin{aligned}
\operatorname{vol}(K / M) \int_{\mathfrak{a}_{\mathfrak{s}}} \prod_{\lambda \in \Sigma_{+}}|(\lambda, \nu)| \int_{K} \int_{\mathfrak{s}} f(1, X) e^{i\langle\operatorname{Ad} k \cdot \nu \mid X\rangle} d X d k d \nu \\
=\left[W_{\mathfrak{s}}\right] \int_{\mathfrak{s}^{\vee}} \int_{\mathfrak{s}} f(1, X) e^{i\langle\alpha \mid X\rangle} d X d \alpha \\
=\left[W_{\mathfrak{s}}\right](2 \pi)^{\operatorname{dim} \mathfrak{s}} f(1,0) . \quad
\end{aligned}
$$

REMARK 29. The constant $\gamma\left(P_{0}\right)$ appears in Harish-Chandra's Plancherel formula [H-CIII, Theorem 27.3]. It can be computed, as in [W2, p. 324], using the Gindikin Karpelevic technique. (Note that the Riemannian normalization of Haar measures satisfies the precondition [W2, p. 320, †]. The rank one case can be handled by noting that $k M_{\lambda} \mapsto \operatorname{Ad} k \cdot H_{\lambda}$ identifies $K_{\lambda} / M_{\lambda}$ with the sphere of radius $1 /(\lambda \mid \lambda)$ in $\mathfrak{s}_{\lambda}$, where $H_{\lambda} \in \mathfrak{a}_{\mathfrak{s}} \cap \mathfrak{s}_{\lambda}$ and $\lambda\left(H_{\lambda}\right)=1$.) I may as well record the other constants.

Let $P=M A N$ be a standard parabolic subgroup of the reductive group $G$ and let $\Sigma(P)$ be the set of roots of $\mathfrak{a}$ in $\mathfrak{n}$ (where $\mathfrak{a}$ is the Lie algebra of $A$, etc.). For $\alpha \in \Sigma(P)$ define

$$
\begin{aligned}
& \Sigma_{+}(\alpha)=\left\{\lambda \in \Sigma_{+}: \lambda \mid \mathfrak{a}=k \alpha \text { with } k>0\right\}, \\
& \rho_{\alpha}=\frac{1}{2} \sum\left\{m(\lambda) \lambda: \lambda \in \Sigma_{+}(\alpha)\right\}, \\
& \Sigma_{P}^{+}=\bigcup_{\alpha \in \Sigma(P)} \Sigma_{+}(\alpha), \\
& \Gamma(\lambda, \nu)=\Gamma\left(\frac{1}{4} m\left(\frac{1}{2} \lambda\right)+\nu_{\lambda}\right) / \Gamma\left(\frac{1}{2} m(\lambda)+\frac{1}{4} m\left(\frac{1}{2} \lambda\right)+\nu_{\lambda}\right),
\end{aligned}
$$

where $\nu \in \mathfrak{a}_{\mathfrak{s} c}^{\vee}$ and $\nu_{\lambda}=(\nu, \lambda) /(\lambda, \lambda)$ and $\lambda \in \Sigma_{+}$. Then in the notation of [H-CIII, p. 125]

$$
\begin{aligned}
\gamma(P) & =\gamma(G / A)=\gamma\left(P_{0}\right) / \gamma\left({ }^{*} P_{0}\right)=(\sqrt{2})^{-\operatorname{dim}\left(K / K_{P}\right)} \operatorname{vol}\left(K / K_{P}\right) \\
& =\prod_{\lambda \in \Sigma_{P}^{+}} \Gamma\left(\lambda, \rho_{\mathrm{s}}\right)(2 \pi /(\lambda, \lambda))^{m(\lambda) / 2} .
\end{aligned}
$$

Similarly using the notation of [H-CIII, p. 139]

$$
\begin{aligned}
c(P) & =c(G / A)=\gamma_{\bar{P} \mid P} \gamma(P)^{-1}=\gamma(P)^{-1} \prod_{\alpha \in \Sigma(P)} \gamma\left(M_{\alpha} / A\right) \\
& =\prod_{\alpha \in \Sigma(P)} \prod_{\lambda \in \Sigma_{+}(\alpha)} \Gamma\left(\lambda, \rho_{\alpha}\right) / \Gamma\left(\lambda, \rho_{\mathfrak{s}}\right)
\end{aligned}
$$

(where $M_{\alpha}$ is the centralizer of ker $\alpha \subseteq \mathfrak{a}$ in $G$ ).

5. Asymptotics. Our next goal is to obtain a description of the asymptotic behavior of spherical functions on Cartan motion groups, and of the Eisenstein 
integrals

$$
E(\Lambda, x) \cdot v=\int_{K} \mu\left(k^{-1}\right) \cdot v e^{\Lambda H(\operatorname{Ad} k \cdot x)} d k
$$

for $x \in \mathfrak{s} \subseteq H$ and complex $\Lambda \in \mathfrak{a}_{\mathfrak{s c}}^{\vee}$. The technique is a variation of the method of stationary phase, but not like that in $[\mathbf{M}]$. Here we conceive of the phase function as being the real vector valued function $k \mapsto H(\operatorname{Ad} k \cdot): K \rightarrow \mathscr{L}\left(\mathfrak{a}_{\mathfrak{s}}\right)$ and think of $\Lambda, h$ as several (complex) parameters replacing the usual real parameter $t$ [BC, Theorem 3.1]. A similar result in the zonal case and with regular $\Lambda$ and $h$ is given in [BC, Theorems 3.9 and 4.3 ].

Fix an ordering on the roots of the reductive group $G$ associated to $H$ relative to our choice of $\mathfrak{a}_{\mathfrak{s}} \subseteq \mathfrak{s}$. Let $P=L U$ be a standard parabolic subgroup of $G$. Reverting to the notation around Corollary 20, let $\Sigma_{L}$ be the roots of $\mathfrak{a}_{\mathfrak{s}}$ in the Levi component $L$ of $P$, and let $\Sigma_{P}^{+}$be the roots in the unipotent radical $U$. Call $h \in \mathfrak{a}_{\mathfrak{s} c}$ dominant if $\mathfrak{R} \lambda(h) \geq 0$ and $\mathfrak{I} \lambda(h) \geq 0$ when $\mathfrak{R} \lambda(h)=0$ (for all positive roots $\lambda$ ). (Here $\mathfrak{R}$ and $\mathfrak{I}$ denote the real and imaginary parts respectively.) For real $a>0$ define the set

$$
\begin{aligned}
\mathscr{C}(P, a)=\left\{h \in \mathfrak{a}_{\mathfrak{s} c}: h \text { is dominant, and } \lambda(h)=0 \text { for } \lambda \in \Sigma_{L},\right. \text { and } \\
\left.\qquad|\lambda(h)| \geq a|h|>0 \text { for } \lambda \in \Sigma_{P}^{+}\right\} .
\end{aligned}
$$

Then every point of $\mathfrak{a}_{\mathfrak{s} c}$ is conjugate by $W_{\mathfrak{s}}$ to a point of $\mathscr{C}(P, a)$ for some (unique) choice of $P$ and some $a>0$. If $\Lambda: \mathfrak{a}_{\mathfrak{s}} \rightarrow \mathbf{C}$ is real linear, write $\Lambda \in \mathscr{C}(P, a)$ if $H_{\Lambda} \in \mathscr{C}(P, a)$. Also set $\mathscr{C}_{0}(P, a)=\mathscr{C}(P, a) \cap \mathfrak{a}_{\mathrm{s}}$. Since

$$
E(w \cdot \Lambda, \operatorname{Ad} k \cdot x) \cdot v=\mu(k) E(\Lambda, x) \cdot \mu\left(w^{-1}\right) v,
$$

it will suffice to obtain asymptotic estimates for $x=h \in \mathscr{C}_{\circ}(P, a)$ and $\Lambda \in \mathscr{C}(Q, b)$. For fixed $P$ and $Q$ and for $w \in W_{Q} \backslash W_{\mathfrak{s}} / W_{P}$ define $\Theta_{w}=\left\{\lambda \in \Sigma_{P}^{+} \mid w \cdot \lambda \in \pm \Sigma_{Q}^{+}\right\}$.

LEMma 30. Let $h \in \mathscr{C}_{0}(P, a)$ and $\Lambda \in \mathscr{C}(Q, b)$ and let $g \in C^{\infty}(K)$. Recalling the notation $K_{P}=K \cap P$, define

$$
\bar{g}(k)=\int_{K_{P}}\left(\int_{K_{Q}} g(m k l) d m\right) d l .
$$

Then there is an asymptotic expansion

$$
\begin{aligned}
\int_{K} g(k) e^{\Lambda H(\operatorname{Ad} k \cdot h)} d k \\
=\sum_{w \in W_{Q} \backslash W_{\mathfrak{s}} / W_{P}} c_{w} e^{\langle\Lambda \mid w \cdot h\rangle} \prod_{\lambda \in \Theta_{w}}\left(\lambda(h) w \cdot \lambda\left(H_{\Lambda}\right)\right)^{-m(\lambda) / 2} \\
\quad \times\left\{\bar{g}(w)+\sum_{1 \leq|I| \leq 3 N} \frac{p_{I}(\bar{g}, \Lambda, h)}{\left(\lambda(h) w \cdot \lambda\left(H_{\Lambda}\right)\right)^{I}}\right\}
\end{aligned}
$$

in the sense that the difference is dominated by $\sigma(g)(|\Lambda||h|)^{-N-N_{w} / 2-1} e^{\langle\Re \Lambda \mid h\rangle}$ (where $N_{w}=\sum_{\lambda \in \Theta_{w}} m(\lambda)$ and $\sigma$ is a seminorm on $C^{\infty}(K)$ of order $\leq$ $\left.3\left(N+N_{w} / 2+1\right)\right)$, this for each integer $N \geq 0$. Here $I$ is a multi-index defined on $\Theta_{w}$ and the $p_{I}(\bar{g}, \Lambda, h)$ are polynomials in $\Lambda, h$ of degree $\leq 2|I| / 3$. Each $p_{I}(\bar{g}, \Lambda, h)$ is a sum of terms homogeneous of degree $k$ (say) simultaneously in $\Lambda$ 
and $h$ with coefficients of the form $D_{k} \bar{g}(w)$ for some differential operators $D_{k}$ of order $\leq 2|I|-3 k$. The square root is computed as the product of the branches of the square roots $\left(\lambda(h) w \cdot \lambda\left(H_{\Lambda}\right)\right)^{1 / 2}$ with arguments satisfying $-\pi / 2<\arg \leq \pi / 2$. Finally the constants $c_{w}$ are given by

$$
c_{w}=\frac{(2 \pi)^{N_{w} / 2} \operatorname{vol}\left(K_{P}\right) \operatorname{vol}\left(K_{Q}\right)}{\operatorname{vol}(K) \operatorname{vol}\left(K_{P} \cap w^{-1} K_{Q} w\right)}
$$

where the volumes are computed relative to the Riemannian measures.

REMARK 31. Part of the assertion of the lemma is the uniformity in $\Lambda$ and $h$ of the asymptotic approximation. If $w \in W_{Q} \backslash W_{\mathrm{s}} / W_{P}$ does not lie in the double coset of 1 , then it may very well be that $\mathfrak{R}\langle\Lambda \mid w h-h\rangle$ is negative, and if this is of the order of magnitude of $|\Lambda||h|$, then the part of the asymptotic expansion corresponding to $w$ will be of order $(|\Lambda||h|)^{-N} e^{\langle\Re \Lambda \mid h\rangle}$ for all $N$. That is, it will be swamped by the error term. However, if you assume that

$$
\mathfrak{R}\langle\Lambda \mid w h-h\rangle \geq-C(\log |\Lambda||h|)^{k} \quad(C>0)
$$

for some $k$, then the part corresponding to $w$ contributes significantly to the asymptotic expansion whenever $N>k$. A straightforward application of the method of stationary phase (as given in $[\mathbf{M}]$ ) would not yield this result. It would force the real part to go to infinity at the same rate as $|\Lambda||h|$, and hence force to zero any contribution corresponding to $w$ with $\mathfrak{R}\langle\Lambda \mid w h\rangle<\mathfrak{R}\langle\Lambda \mid h\rangle$. (See [BC, Theorem 4.3].) Divisibility problems prevent us from getting such a clean formula as in [BC, Theorem 3.9]. The differential operators mentioned in the lemma are described more fully in step (8) of the proof.

PROOF. We wish to estimate

$$
I(g, \Lambda, h)=\int_{K} g(k) e^{\Lambda H(\operatorname{Ad} k \cdot h)} d k .
$$

(1) First let us set up some notation. For the phase function, write

$$
\phi(\Lambda, k, h)=\Lambda H(\operatorname{Ad} k \cdot h) .
$$

Replace $\exp (x)=e^{x}$. Let $\mathfrak{y}$ be the orthogonal complement of $\mathfrak{m}$ in $\mathfrak{k}$ and let $\mathfrak{y}=\sum \mathfrak{y}_{\lambda}$ be the orthogonal decomposition as described at the beginning of $\S 3$. Writing $Y=\sum Y_{\lambda}$ accordingly, set $|Y|_{\lambda}^{2}=-B\left(Y_{\lambda}, Y_{\lambda}\right)$. Define the quadratic form

$$
q(h, \Lambda, Y)=\sum_{\lambda \in \Sigma^{+}} \lambda(h) \lambda\left(H_{\Lambda}\right)|Y|_{\lambda}^{2} .
$$

(2) The structure of the critical set for the phase function $k \mapsto \phi(\Lambda, k, h)$ is given in [DKV, p. 316]. The critical set is $\coprod\left\{K_{Q} w K_{P} \mid w \in W_{Q} \backslash W_{\mathfrak{s}} / W_{P}\right\}$ (where $W_{P}$ and $W_{Q}$ are the centralizers of $\mathscr{C}_{\circ}(P, a)$ and $\mathscr{C}(Q, b)$ in $W_{\mathrm{s}}$ respectively). The Hessian on $K_{Q} w l \subseteq K_{Q} w K_{P}$ is given by $-q\left(h, w^{-1} \Lambda, \operatorname{Ad} l \cdot Y\right)$. (Notice that $K_{P}=K_{P}^{\circ} \cdot M$ and the Lie algebra of $K_{P}$ is spanned by $\mathfrak{m}$ and the $\mathfrak{y}_{\lambda}$ such that $\lambda(h)=0$. Thus $K_{P}$ is the centralizer of any particular $h \in \mathscr{C}(P, a)$. Also $W_{P}$ is generated by the reflections in the roots which vanish on $h$, so $W_{P}$ is the Weyl group of the Levi component of $P$. Similar facts hold for $K_{Q}$ and $W_{Q}$. Thus the critical set depends only on $P$ and $Q$ with $h \in \mathscr{C}_{\circ}(P, a)$ and $\Lambda \in \mathscr{C}(Q, b)$, as the notation suggests.)

Similar results hold for $\mathfrak{R} \phi(\Lambda, k, h)=\phi(M, k, h)$ (where $M$ is the real part of $\Lambda)$. For the moment write $K_{M}$ and $W_{M}$ for the centralizer of $M$ in $K$ and $W_{\mathfrak{s}}$ 
respectively. Below we show that the Hessian of $\phi(M, k, h)$ is negative semidefinite on $K_{M} w K_{P}$ if and only if $w$ lies in the double coset of 1 in $W_{M} \backslash W_{\mathfrak{s}} / W_{P}$. This implies that the maximum of the real part of the phase function is $e^{\langle\Lambda \mid h\rangle}$ and occurs precisely on $K_{M} 1 K_{P}$. As we will see, this accounts for the exponential factor in the error term.

To see the assertion about negative semidefiniteness, it suffices to find a positive root $\beta$ such that $\beta(h)>0$ and $\beta\left(w^{-1} M\right)<0$, assuming that $w \notin W_{M} \cdot 1 \cdot W_{P}$. Since $h$ and $M$ lie in the closed positive Weyl chamber, this gives $\beta>0$ and $w \cdot \beta<0$. Suppose $w$ has the shortest length of any element in its double coset $W_{M} \cdot w \cdot W_{P}$, and let $w=w_{1} \ldots w_{l}$ be a shortest word for $w$ in terms of reflections $w_{k}$ in the simple root $\alpha_{k}$. The positive roots which are made negative by $w$ are of the form $\beta_{k}=w_{l} \ldots w_{k+1} \cdot \alpha_{k}$. Now $w$ is also the shortest element of its coset $w \cdot W_{P}$. If $0=\beta_{k}(h)=\alpha_{k}\left(w_{k+1} \ldots w_{l} \cdot h\right)$ then $w_{k}$ centralizes $w_{k+1} \ldots w_{l} \cdot h$ and so $w_{1} \ldots w_{k-1} w_{k+1} \ldots w_{l}$ is a shorter element of $w \cdot W_{P}$. This shows that $\beta_{k}(h) \neq 0$, and so $\beta_{k}(h)>0$ since $h$ lies in the closed Weyl chamber. Similarly the equation $w \cdot \beta_{k}(M)=-\alpha_{k}\left(w_{k-1} \ldots w_{1} \cdot M\right)=0$ yields a shorter element of $W_{M} \cdot w \cdot W_{P}$. Thus any $\beta>0$ with $w \cdot \beta<0$ fulfills our requirements.

(3) We generalize the statement we are trying to prove, eliminating extraneous details. Let $s$ be a parameter varying in a compact set $S$. Assume only that $K$ is a smooth manifold equipped with a measure $d k$ which is locally a positive $C^{\infty}$ multiple of Lebesgue measure. Assume that the phase function is of the form

$$
\phi(\Lambda, s, k)=\langle\Lambda \mid A(s, k)\rangle
$$

for some smooth function $A: S \times K \rightarrow \mathfrak{a}$, where $\mathfrak{a}$ is a real vector space and $\Lambda: \mathfrak{a} \rightarrow \mathbf{C}$ is real linear. (Here $\Lambda$ replaces $\Lambda \otimes h$ in the statement of the lemma.) Assume that the critical set $K_{\phi}$ is a manifold which is independent of $s$ and $\Lambda$, and that the Hessian is nondegenerate transversally to the critical set, for all $s \in S$ and all $\Lambda \in \mathscr{C}$, where $\mathscr{C} \subseteq \mathfrak{a}_{c}^{\vee} \backslash\{0\}$ is a closed conical set. (Note that $\mathscr{C}=\{\Lambda \otimes h \mid \Lambda \in \mathscr{C}(Q, b)$ and $\left.h \in \mathscr{C}_{0}(P, a)\right\}$ is a closed conical set in $\left.\mathfrak{a}_{\mathfrak{s} c}^{\vee} \otimes \mathfrak{a}_{\mathfrak{s}} \backslash\{0\}.\right)$

Let $\mathscr{K}$ be the set of components of $K_{\phi}$, so the phase function $k \mapsto\langle\Lambda \mid A(s, k)\rangle$ is constant on each $C \in \mathscr{K}$. Then I claim that there is a measure $d \mu_{C}(\Lambda, s)$ on each $C$, which is smooth in $s$ and holomorphic and homogeneous of degree $-d / 2=-(\operatorname{dim} K-\operatorname{dim} C) / 2$ in $\Lambda$, such that we have the asymptotic expansion

$$
\int_{K} g(k) e^{\langle\Lambda \mid A(s, k)\rangle} d k \sim \sum_{C \in \mathscr{K}} e^{\langle\Lambda \mid A(s, C)\rangle} \int_{C} \sum_{m=0}^{3 N} D_{m}(\Lambda, s, u) g(u) d \mu_{C}(\Lambda, s, u)
$$

for any $g \in C_{c}^{\infty}(K)$. This means that the difference is dominated by

$$
\sigma(g)|\Lambda|^{-N-d / 2-1} e^{\delta(\Re \Lambda)}
$$

where $\sigma$ is a seminorm on $C_{c}^{\infty}(K)$ (of order $\leq 3(N+d / 2+1)$ ) and $\delta(\Re \Lambda)$ is the supremum of $\langle\mathfrak{R} \Lambda \mid A(s, k)\rangle$ for $s \in S$ and $k$ in the support of $g$. The $D_{m}(\Lambda, s, u)$ are differential operators on $K$ of degree $2 m$ which are rational functions of $\Lambda$, a sum of operators homogeneous of degrees between $-m / 3$ and $-m$ (and smooth in $s$ ).

(4) Let $g \in C_{c}^{\infty}(K)$ and suppose that $g$ vanishes to order $2 N$ on the critical set $K_{\phi}$ (i.e., all derivitives of order $\leq 2 N-1$ of $g$ are zero on $K_{\phi}$ ). Then we show that

$$
\left|\int_{K} g(k) e^{\langle\Lambda \mid A(s, k)\rangle} d k\right| \leq \sigma(g)|\Lambda|^{-N} e^{\delta(\Re \Lambda)}
$$


where $\sigma$ is a seminorm of order $\leq 3 N$ on $C_{c}^{\infty}(K)$ and $\delta(\Re \Lambda)$ is as in step (3).

To see this, let $X_{i}$ be a finite collection of vector fields on $K$ which span the tangent space at each point of a neighborhood $U$ of the support of $g$. Set

$$
a_{i}=X_{i} \phi(\Lambda /|\Lambda|, s, k)=\left\langle\Lambda \mid X_{i} A(s, k)\right\rangle /|\Lambda|,
$$

and define $a=\sum\left|a_{i}\right|^{2}$. Then $a$ vanishes to order two on $K_{\phi}$ and nowhere else in $U$. Define the vector field $X$ on $U \backslash K_{\phi}$ by $X=\sum_{i}\left(\bar{a}_{i} / a\right) X_{i}$ (where bar is complex conjugate). Then

$$
X \cdot \exp (\phi(\Lambda, s, k))=|\Lambda| \exp (\phi(\Lambda, s, k)) .
$$

Let $X_{i}^{*}=Y_{i}+\beta_{i}$ be the formal adjoint of $X_{i}$ with respect to $d k$, where $Y_{i}$ is a vector field and $\beta_{i}$ is a function, so

$$
X_{i}^{*}\left(\bar{a}_{i} f / a^{p+1}\right)=\left\{a Y_{i}\left(\bar{a}_{i} f\right)-(p+1) \bar{a}_{i} f Y_{i}(a)+a \beta_{i} \bar{a}_{i} f\right\} / a^{p+2}
$$

where the numerator vanishes to order $n+2$ on $K_{\phi}$ if $f$ vanishes to order $n$ there. Thus by induction, $\left(X^{*}\right)^{N} \cdot g=f / a^{2 N}$ off $K_{\phi}$, where the numerator vanishes to order $4 N$ on $K_{\phi}$ and involves derivatives of $g$ of order $\leq N$.

Locally we can take $K_{\phi} \subseteq K$ to be the inclusion $\mathbf{R}^{m} \subseteq \mathbf{R}^{m} \times \mathbf{R}^{n}$ of Euclidean spaces with standard coordinates $\left(u_{1}, \ldots, u_{m}, x_{1}, \ldots, x_{n}\right)$. Replacing $g$ by its $2 N$ th order Taylor remainder

$$
g(u, x)=\frac{1}{(2 N-1) !} \sum_{|I|=2 N} x^{I} \int_{0}^{1}(1-t)^{2 N-1} \frac{\partial^{|I|} g}{\partial x^{I}}(u, t x) d t
$$

and $\bar{a}_{i}$ and $a$ by their first and second order remainders, respectively, gives

$$
\left(X^{*}\right)^{N} \cdot g(u, x)=\frac{1}{a^{2 N}}\left(\sum_{|I|=4 N} x^{I} g_{I}(u, x)\right)
$$

where the coefficients $g_{I}$ are $C^{\infty}$ functions constructed from derivatives of $g$ of order $\leq 3 N$.

Still working locally, we may suppose the $X_{i}$ are renumbered so that

$$
X_{i}=\sum c_{i j} \frac{\partial}{\partial x_{j}}+\sum b_{i j} \frac{\partial}{\partial u_{j}}
$$

where $c_{i j}$ for $i, j=1, \ldots, n$ is an invertible matrix of smooth functions on $U$. Taking the first order Taylor expansion of $a_{i}$ gives

$$
a_{i}(u, x)=\sum_{j, k} x_{k} c_{i j}(u, 0) H_{j k}(u, 0)+R_{i}
$$

where $H_{j k}(u, 0)$ is the Hessian matrix of $\phi$, assumed nonsingular on $\mathbf{R}^{m}\left(=K_{\phi}\right)$ and the remainder $R_{i}$ vanishes to second order on $\mathbf{R}^{m}$. This gives

$$
\begin{aligned}
a(u, x) & \geq \sum_{i=1}^{n}\left|c_{i j} H_{j k} x_{k}\right|^{2}-R+\sum_{i>n}\left|a_{i}\right|^{2} \\
& \geq C|x|^{2} \quad(C>0)
\end{aligned}
$$

for $x$ near zero. But now if $|I|=4 N$ then $\left|x^{I} / a^{2 N}\right| \leq C^{-2 N}$, so $x^{I} / a^{2 N}$ are bounded near $K_{\phi}$. 
Now choose a finite partition of unity on $S \times\{\Lambda \in \mathscr{C}:|\Lambda|=1\} \times U$ which is fine enough so that the above local calclations are valid. Then

$$
\begin{aligned}
|\Lambda|^{N}\left|\int_{K} g(k) e^{\langle\Lambda \mid A(s, k)\rangle} d k\right| & \leq \int_{K}\left|\left(X^{*}\right)^{N} \cdot g(k)\right| e^{\Re\langle\Lambda \mid A(s, k)\rangle} d k \\
& \leq e^{\delta(\Re \Lambda)} \int_{K}\left|\left(X^{*}\right)^{N} \cdot g(k)\right| d k .
\end{aligned}
$$

Breaking apart this final integral according to the partition of unity, and observing that the coefficients of $X$ and all of their derivatives with respect to $k$ can be bounded uniformly in $s$ and $\Lambda$ when $k$ stays outside of a neighborhood of $K_{\phi}$, completes the proof of the assertion of step (4). This says that we can replace $g$ by its $2 N$ th order Taylor series, locally (times a cutoff function), to achieve an error term of order $N$, and then subsequent analysis will not increase the order $3 N$ of $\sigma$.

(5) By step four, we can work locally near $K_{\phi}$, and suppose we are dealing with $\mathbf{R}^{m} \subseteq \mathbf{R}^{m} \times \mathbf{R}^{n}$. Write $d k=J(u, x) d u d x$ and let $g \in C_{c}^{\infty}\left(\mathbf{R}^{m} \times \mathbf{R}^{n}\right)$ be supported near zero. Factoring the constant $e^{\langle\Lambda \mid A(s,(u, 0))\rangle}$ out of the integral, it suffices to obtain the asymptotic expansion of

$$
I(g, \Lambda)=\iint g(u, x) J(u, x) e^{\langle\Lambda \mid A(s, u, x)\rangle} d u d x
$$

where now we assume

$$
A(s, u, x)=-q(s, u, x)+h(s, u, x)
$$

and

$$
-q(s, u, x)=\frac{1}{2}\left\langle\Lambda \mid \sum_{i, j=1}^{n} H_{i j}(s, u) x_{i} x_{j}\right\rangle
$$

is a nondegenerate quadratic form in $x$, and $h$ vanishes to order three along $\mathbf{R}^{m}$.

Suppressing variables and using a Taylor expansion on $e^{\langle\Lambda \mid h\rangle}$, we get

$$
\begin{aligned}
I(g, U)= & \sum_{k=0}^{M} \frac{1}{k !} \iint g J\langle\Lambda \mid h\rangle^{k} e^{-q} d u d x \\
& +\int_{0}^{1} \frac{1}{M !}(1-t)^{M} \iint g J\langle\Lambda \mid h\rangle^{M+1} e^{-q+t h} d u d x d t .
\end{aligned}
$$

Take $M$ odd and set $M+1=2 N$. Now $\langle\Lambda \mid h\rangle^{M+1}$ is a polynomial in $\Lambda$ homogeneous of degree $2 N$ with coefficients which vanish to order $6 N$ on $\mathbf{R}^{m}$. Since the differential $d q$ vanishes only on $\mathbf{R}^{m}$ to order one and $d h$ vanishes to order two along $\mathbf{R}^{m}$, we may assume that the critical set of the new phase function, $-q(s, u, x)+\operatorname{th}(s, u, x)$, intersects the support of $g$ only in $\mathbf{R}^{m}$. The Hessian along $\mathbf{R}^{m}$ is essentially $-2 q$, assumed nondegenerate. Thus step (4) implies that the remainder integral in this Taylor expansion is dominated by

$$
\left.C|\Lambda|^{2 N} \cdot \sigma(g)|\Lambda|^{-3 N} e^{\delta_{1}(\Re \Lambda)} \quad \text { (some } C>0\right) .
$$

The contribution to the error in the entire integral is

$$
C \sigma(g)|\Lambda|^{-N} e^{\langle\Re \Lambda \mid A(s, u, 0)\rangle+\delta_{1}(\Re \Lambda)} .
$$


Here $g$ may be assumed to be supported arbitrarily close to $\mathbf{R}^{m}$, so we may take $\delta_{1}(\Re \Lambda)$ quadratically small in the distance from $\mathbf{R}^{m}$. Moreover it is easy to check that we may assume $\delta_{1}(\Re \Lambda) \leq 0$ when $\langle\Re \Lambda \mid-q+h\rangle \leq 0$. Thus the new error term is of the same or smaller order of magnitude as that of step (4), so we can retain the error term of step (4).

So now we may assume that the phase function is quadratic in $x$ (and linear in $\Lambda$ ). Also step (4) implies that we can replace the amplitudes $g J\langle\Lambda \mid h\rangle^{k}$ by their Taylor series in $x$ to order $2 N$ (times a cutoff function in $x$ ). The point is that the integrand may be extended holomorphically to a neighborhood of $\mathbf{R}^{m}$ in $\mathbf{R}^{m} \times \mathbf{C}^{n}$. Thus if $z_{1}, \ldots, z_{n}$ are the coordinates on $\mathbf{C}^{n}$, we can compute our integral as the integral of a differential form of type $(n, 0)$

$$
\int_{\mathbf{R}^{m} \times \mathbf{R}^{n}} \alpha(z) p(s, u, z) e^{-q(s, u, z)} d z_{1} \wedge \cdots \wedge d z_{n} d u
$$

where $\alpha \in C_{c}^{\infty}\left(\mathbf{C}^{n}\right)$ is identically one in a neighborhood of the origin and $p$ is a Taylor polynomial.

(6) Let $Q(\Lambda)=Q(\Lambda, s, u)$ be the matrix of the quadratic form $q$. We show that if $Q(\Lambda, s, u)$ has a negative real eigenvalue at any point $u$ of $\mathbf{R}^{m}$ in the support of $g$, then the part of the integral coming from a neighborhood of $(s, u)$ does not contribute to the asymptotic expansion. If $Q(\Lambda)$ has a negative real eigenvalue then $Q(\Lambda /|\Lambda|)=Q(\Lambda) /|\Lambda|$ does too; call it $-\mu$. Let $x+i y \in \mathbf{C}^{n}$ be the corresponding eigenvector. Then

$$
\langle Q(\Lambda) x \mid x\rangle+\langle Q(\Lambda) y \mid y\rangle=\langle Q(\Lambda)(x+i y) \mid x-i y\rangle=-\mu|\Lambda|(\langle x \mid x\rangle+\langle y \mid y\rangle) .
$$

This shows that we may choose $x$ as close as we please to $\mathbf{R}^{m}$ and in the support of $g$ such that $\Re q(\Lambda, s, u, x) \leq-C|\Lambda|$ for some constant $C>0$. But then the original phase function satisfies

$$
\delta(\mathfrak{R} \Lambda) \geq \mathfrak{R} \phi(\Lambda, s, u, x) \geq \mathfrak{R}\langle\Lambda \mid A(s, u, 0)\rangle+C|\Lambda| .
$$

Thus the contribution to the asymptotic expansion near $(s, u, 0)$ will be of order

$$
e^{\delta(\Re \Lambda)} e^{-C|\Lambda| / 2}=\mathscr{O}\left(|\Lambda|^{-N}\right) e^{\delta(\Re \Lambda)}
$$

for all $N$, hence can be ignored.

(7) So assume $Q(\Lambda)$ has no negative real eigenvalues. We follow the proof in $[\mathbf{M}$, p. 152]. For $0 \leq t \leq 1$ set $Q(\Lambda, t)=t \cdot 1+(1-t) Q(\Lambda /|\Lambda|, s, u)$. Then $Q(\Lambda, t)$ is a nonsingular symmetric matrix with no negative real eigenvalues for all $t$. On C slit along the negative real axis, let $w^{1 / 2}$ be the branch of the square root with argument satisfying $-\pi / 2<\arg <\pi / 2$. Use the usual method, of integrating $(Q(\Lambda, t)-w \cdot 1)^{-1} w^{1 / 2}$ around a large contour, to define the square root $Q(\Lambda, t)^{1 / 2}$ which is symmetric and continuous in $u, s$ and $\Lambda /|\Lambda|$. I wish to make the change of variable $z \mapsto Q(\Lambda, 0)^{-1 / 2} \cdot z$ in the last integral in step (5). So let

$$
\omega(u, z)=p(s, u, z) e^{-q(s, u, z)} d z_{1} \wedge \cdots \wedge d z_{n},
$$

holomorphic in $z$.

Define a chain $\Gamma_{u}=\Gamma_{\Lambda, s, u}$ in $\mathbf{C}^{n}$ by $\Gamma_{u}(t, x)=Q(\Lambda, t)^{-1 / 2} \cdot x$. Since $Q(\Lambda, 1)^{-1 / 2}$ $=1$, the integral in (5) is $\iint_{\Gamma_{u}(1, \cdot)} \alpha \omega d u$, and since ${ }^{t} Q(\Lambda, 0)^{-1 / 2} Q(\Lambda) Q(\Lambda, 0)^{-1 / 2}$ 
$=|\Lambda| \cdot 1$ we have

(*) $\iint_{\Gamma_{u}(0, \cdot)} \alpha \omega d u$

$$
=\operatorname{det}\left(Q^{-1 / 2}\right) \iint \alpha\left(Q^{-1 / 2} \cdot x\right) p\left(s, u, Q^{-1 / 2} \cdot x\right) e^{-|\Lambda|\langle x \mid x\rangle} d x d u
$$

where $Q^{-1 / 2}=Q(\Lambda /|\Lambda|)^{-1 / 2}$. (Here $\operatorname{det}\left(Q^{-1 / 2}\right)$ is the constant Jacobian determinant for the transformation $x \mapsto Q(\Lambda, 0)^{-1 / 2} \cdot x$.) On the other hand Stokes' theorem gives

$$
\left|\iint_{\Gamma_{u}(1, \cdot)} \alpha \omega d u-\iint_{\Gamma_{u}(0, \cdot)} \alpha \omega d u\right|=\left|\iint_{\Gamma_{u}}(\bar{\partial} \alpha) \omega d u\right| \leq \sigma(g)|\Lambda|^{-M} e^{\delta(\Re \Lambda)}
$$

for all $M$. The estimate follows as in step (4) from the fact that $\bar{\partial} \alpha$ vanishes in a neighborhood of the critical set and as in step (5) from the fact that

$$
\mathfrak{R}\left\langle Q(\Lambda) Q(\Lambda, t)^{-1 / 2} \cdot x \mid Q(\Lambda, t)^{-1 / 2} \cdot x\right\rangle \geq 0 \text { when } \mathfrak{R}\langle Q(\Lambda) \cdot x \mid x\rangle \geq 0 .
$$

So now it suffices to estimate the integral $(*)$. But this is well known and easy. First we will have a factor

$$
|\Lambda|^{-1 / 2} \operatorname{det} Q(\Lambda /|\Lambda|)^{-1 / 2}=\operatorname{det} Q(\Lambda)^{-1 / 2}
$$

where the branch of the square root is determined as the product of the square roots of the eigenvalues with argument between $-\pi / 2$ and $\pi / 2$. The summands will involve the Laplacian

$$
\begin{aligned}
\frac{1}{|\Lambda|} \sum & \frac{\partial^{2}}{\partial x_{i}^{2}}\left(x \mapsto p\left(s, u, Q^{-1 / 2} \cdot x\right)\right)_{x=0} \\
\quad & =\left.\frac{1}{|\Lambda|} \sum Q(\Lambda /|\Lambda|)_{j i}^{-1 / 2} Q(\Lambda /|\Lambda|)_{k i}^{-1 / 2} \frac{\partial}{\partial z_{j}} \frac{\partial}{\partial z_{k}} p(s, u, z)\right|_{z=0} \\
\quad & \left.\left(\sum Q(\Lambda)_{j k}^{-1} \frac{\partial}{\partial x_{j}} \frac{\partial}{\partial x_{k}}\right) p(s, u, x)\right|_{x=0}
\end{aligned}
$$

where we have used the Cauchy-Riemann equations twice, and the symmetry of $Q^{-1 / 2}$. Let $\left\langle Q(\Lambda)^{-1} \partial / \partial x \mid \partial / \partial x\right\rangle$ denote this last differential operator. Then the asymptotic expansion of $(*)$ is

$$
\begin{aligned}
& \left.\pi^{\operatorname{codim} K_{\phi} / 2} \int \operatorname{det} Q(\Lambda)^{-1 / 2} \sum_{m=0}^{\operatorname{deg} p / 2} \frac{1}{m !}\left\langle\frac{1}{4} Q(\Lambda)^{-1} \frac{\partial}{\partial x} \mid \frac{\partial}{\partial x}\right\rangle^{m} p(s, u, x)\right|_{x=0} d u \\
& +o\left(e^{-\delta^{2}|\Lambda|}\right)
\end{aligned}
$$

(where $\delta>0$ is a constant depending on the size of the ball where $\alpha \equiv 1$ ).

Let us unwind all of this. Recall that $p$ was a Taylor polynomial for $g J\langle\Lambda \mid h\rangle^{k}$, which in turn came from a Taylor series for $g J e^{\langle\Lambda \mid h\rangle}$. Thus the derivatives above can be computed as

$$
\left.\left\langle\frac{1}{4} Q(\Lambda)^{-1} \frac{\partial}{\partial x} \mid \frac{\partial}{\partial x}\right\rangle^{m} g(u, x) J(u, x) e^{\langle\Lambda \mid h(s, u, x)\rangle}\right|_{x=0}=D_{m}(\Lambda, s, u) g(u, 0)
$$


(in the notation of step (3)). The $Q(\Lambda)^{-1}$ contribute rational functions in $\Lambda$ homogeneous of degree $-m$ and the derivatives contribute a polynomial in $\Lambda$ of degree $\leq 2 m / 3$ (because $h$ vanishes to order three along $x=0$ ). One may define the measure $\operatorname{det} Q(\Lambda, s, u)^{-1 / 2} d u$ to be zero when $Q(\Lambda)$ has a negative real eigenvalue, or one can define det $Q(\Lambda, s, u)^{-1 / 2}$ as the product of the square roots of the eigenvalues with arguments satisfying $-\pi / 2<\arg \leq \pi / 2$, whichever is convenient. (It does not matter since there will be no contribution to the asymptotic expansion.) Looking back at the claim made in step (3), you will find it has been proved.

(8) We explicate the results of steps (3) and (7) in the context expressed in the lemma, and consider the contribution to the asymptotic expansion coming from the portion $C_{w}=K_{Q} w K_{P}$ of the critical set. Let $\mathfrak{y}_{w}$ be the span of the $\mathfrak{y}_{\lambda}$ (defined at the beginning of $\S 3)$ for $\lambda \in \Theta_{w}=\Sigma_{P}^{+} \cap\left( \pm w^{-1} \Sigma_{Q}^{+}\right)$. Then at each point $k m_{w} l$ of $C_{w}, \operatorname{Ad} l^{-1} \cdot \mathfrak{y}_{w}$ is the orthogonal complement in $\mathfrak{k}$ of the tangent space of the manifold $C_{w}$. Thus we can choose $\delta>0$ such that for $-B(Y, Y)<\delta\left(Y \in \mathfrak{y}_{w}\right)$ the map

$$
\left(k, m_{w}, l, Y\right) \mapsto k m_{w} l \exp \left(\operatorname{Ad} l^{-1} \cdot Y\right)=k m_{w} \exp (Y) l
$$

defines a diffeomorphism of the bundle of balls of radius $\delta$ in this normal bundle onto a neighborhood $U$ of $C_{w}$ in $K$.

In these coordinates, Haar measure on $K$ will be expressed

$$
\int_{K} f(k) d k=\int_{\mathfrak{\eta}_{w}} \bar{f}(Y) J(Y) d Y
$$

where $f$ is supported in $U$ and $J(Y)$ is a Jacobian determinant and

$$
\bar{f}(Y)=\int_{K_{P}} \int_{K_{Q}} f\left(k m_{w} \exp (Y) l\right) d k d l .
$$

The phase function is given in these coordinates by

$$
\begin{aligned}
& \left.\phi\left(\Lambda, k m_{w} \exp (Y) l, h\right)=\langle\Lambda| w \operatorname{Ad}(\exp Y) \cdot h\right) \\
& \quad=\langle\Lambda \mid w \cdot h\rangle-\frac{1}{2} q\left(h, w^{-1} \Lambda, Y\right)+\left\langle w^{-1} \Lambda \mid \sum_{m \geq 3} \frac{1}{m !}(\operatorname{ad} Y)^{m} \cdot h\right\rangle
\end{aligned}
$$

(expressed as in step (4)). Here

$$
q\left(h, w^{-1} \Lambda, Y\right)=\sum_{\lambda \in \Theta_{w}} \lambda(h) w \cdot \lambda\left(H_{\Lambda}\right)|Y|_{\lambda}^{2}
$$

so the differential operator expressed in step (7) is

$$
\left\langle\frac{1}{4} Q(\Lambda)^{-1} \frac{\partial}{\partial x} \mid \frac{\partial}{\partial x}\right\rangle=\sum_{\lambda \in \Theta_{w}}\left(2 \lambda(h) w \cdot \lambda\left(H_{\Lambda}\right)\right)^{-1} \omega_{\lambda}
$$

where $\omega_{\lambda}$ is the Laplacian on $\mathfrak{y}_{\lambda}$. Abbreviate

$$
H(h, \Lambda, Y)=\exp \left\langle w^{-1} \Lambda \mid \sum_{m \geq 3} \frac{1}{m !}(\operatorname{ad} Y)^{m} \cdot h\right\rangle .
$$


Then by step (7), the contribution to the asymptotic expansion coming from $C_{w}$ is

$$
\begin{aligned}
e^{\langle\Lambda \mid w \cdot h\rangle}(2 \pi)^{N_{w} / 2} & \prod_{\lambda \in \Theta_{w}}\left(\lambda(h) w \cdot \lambda\left(H_{\Lambda}\right)\right)^{-m(\lambda) / 2} \\
& \times\left.\sum_{m=0}^{3 N} \frac{1}{m !}\left(\sum\left(2 \lambda(h) w \cdot \lambda\left(H_{\Lambda}\right)\right)^{-1} \omega_{\lambda}\right)^{m} \cdot \bar{g}(Y) J(Y) H(\Lambda, h, Y)\right|_{Y=0} .
\end{aligned}
$$

The rest of the constant $c_{w}$ comes from computing the value of $J(0)$. The volumes can be computed from Remark 29 using

$$
c_{w}=\frac{(2 \pi)^{N_{w} / 2} \operatorname{vol}\left(w^{-1} K_{Q} w / K_{P} \cap w^{-1} K_{Q} w\right)}{\operatorname{vol}\left(K / K_{P}\right)} .
$$

This implies the lemma.

REMARK 32. Notice that $\Lambda$ and $h$ appear in a symmetric manner in

$$
I(g, \Lambda, h)=\int_{K} g(k) e^{\langle\Lambda \mid \operatorname{Ad} k \cdot h\rangle} d k,
$$

so we could just as well have taken $h$ to be complex in Lemma 30 . Then, using the Cauchy integral formula, the asymptotic expansion for the derivatives of $I(g, \Lambda, h)$ with respect to $h$ can be computed by differentiating the asymptotic formula for $I(g, \Lambda, h)$. (This is not always the case for general asymptotic expansions.) Thus the asymptotic expansion will formally satisfy the differential equations corresponding to the radial components of the differential operators in $\mathfrak{X}$. This yields recursion relations satisfied by the $p_{I}$ in Lemma 30 . For example, if $G$ is semisimple and $\Lambda$ and $h$ are regular (and setting $p_{0}(\bar{g}, \Lambda, h)=\bar{g}(w)$ ), then the Laplacian $\omega_{\mathfrak{s}}$ on $\mathfrak{s}$ yields

$$
\begin{array}{r}
\sum_{\lambda \in \Sigma_{+}^{1}}(2 m(\lambda)+8 m(2 \lambda))\left(H_{\lambda}^{2}+2\left\langle\Lambda \mid H_{\lambda}\right\rangle H_{\lambda}\right) \sum_{I} \frac{p_{I}(\bar{g}, \Lambda, h)}{\left(\lambda(h) w \cdot \lambda\left(H_{\Lambda}\right)\right)^{I}} \\
=\sum_{\lambda \in \Sigma_{+}^{1}} \frac{1}{\lambda(h)^{2}} \sum_{I} p_{I}\left(\left(\omega_{\lambda}-\frac{3}{4} \omega_{2 \lambda}+\frac{1}{4}\left(n(\lambda)^{2}-2 n(\lambda)\right)(\lambda \mid \lambda)\right) \cdot \bar{g}, \Lambda, h\right) \\
\times 1 /\left(\lambda(h) w \cdot \lambda\left(H_{\Lambda}\right)\right)^{I}
\end{array}
$$

where $n(\lambda)=m(\lambda)+m(2 \lambda)$ (using Helgason's example below). However, as pointed out in [H2, p. 298], the solution of these recursion relations is problematical, because monomials in the reciprocals of roots fail to be linearly independent. (Helgason's example is

$$
\sum_{\lambda \neq \mu \in \Phi^{+}} \frac{(\lambda \mid \mu)}{\lambda \mu}=\pi_{\Phi}^{-1} \sum H_{i}^{2} \cdot \pi_{\Phi}=0
$$

where $\pi_{\Phi}=\prod_{\lambda \in \Phi^{+}} \lambda$ and $\Phi^{+}$is the positive roots of a semisimple root subsystem $\Phi \subseteq \Sigma^{1}$, so $\pi_{\Phi}$ is harmonic.)

Let us record the form taken by the asymptotic expansions for spherical functions and Eisenstein integrals (but only the leading term since we know so little about the $\left.p_{I}\right)$. 
COROLlary 33. Let $h \in \mathscr{C}_{0}(P, a)$ and $\Lambda \in \mathscr{C}(Q, b)$. Then we have the estimate

$$
\begin{aligned}
E\left(P, \mathfrak{e}, \Lambda, \psi_{T}, h\right) & \sum_{w \in W_{P} \backslash W_{\mathrm{s}} / W_{Q}} c_{w^{-1}} P\left(K_{Q}\right) \mu(w) P\left(K_{P}\right) \cdot v e^{\langle w \cdot \Lambda \mid h\rangle} \\
& \times\left[\prod_{\lambda \in \Theta_{w}}\left(\lambda(h) w \cdot \Lambda\left(H_{\lambda}\right)\right)^{-m(\lambda) / 2}+\mathscr{O}\left((|\Lambda||h|)^{-N_{w}-1}\right)\right]
\end{aligned}
$$

(see (23)) where $P\left(K_{Q}\right)$ is the orthogonal projection onto the subspace which transforms trivially under $K_{Q}$ and other notation is as in Lemma 30. Similarly

$$
\begin{aligned}
& \Phi(\mathfrak{e}, \Lambda, \mathfrak{d}, k h) \cdot l=\sum_{w \in W_{Q} \backslash W_{\mathfrak{s}} / W_{P}} c_{w} l \circ P_{w}\left(\mu_{\mathfrak{o}}(k)\right) e^{\langle\Lambda \mid w \cdot h\rangle} \\
& \quad \times\left[\prod_{\lambda \in \Theta_{w}}\left(\lambda(h) w \cdot \lambda\left(H_{\Lambda}\right)\right)^{-m(\lambda) / 2}+\mathscr{O}\left((|\Lambda||h|)^{-N_{w}-1}\right)\right]
\end{aligned}
$$

where

$$
P_{w}(L)=\int_{K_{P}} \int_{K_{Q}} \mu_{\mathfrak{o}}(m w l) \circ L \circ \mu_{\mathfrak{o}}(m w l)^{-1} d m d l .
$$

We now turn to the Paley-Wiener Theorem. Consider the double $K$-module structure $\tau$ on $C^{\infty}(K \times K)$ defined by

$$
\left(\tau_{1}\left(k_{1}\right) \phi \tau_{2}\left(k_{2}\right)\right)\left(k_{3}, k_{4}\right)=\phi\left(k_{3} k_{1}, k_{2} k_{4}\right) .
$$

Given $f \in C_{c}^{\infty}(H)$ define $F: H \rightarrow C^{\infty}(K \times K)$ by $F(x)\left(k_{1}, k_{2}\right)=f\left(k_{1} x k_{2}\right)$. Then clearly

$$
F\left(k_{1} x k_{2}\right)=\tau_{1}\left(k_{1}\right) F(x) \tau_{2}\left(k_{2}\right)
$$

and conversely any weakly $C_{c}^{\infty} \operatorname{map} F: H \rightarrow C^{\infty}(K \times K)$ satisfying this property comes from some $f \in C_{c}^{\infty}(H)$, namely $f(x)=F(x)(1,1)$.

Let $\Lambda: \mathfrak{s} \rightarrow \mathbf{C}$ be real-linear and consider $\pi=\operatorname{ind}_{\mathfrak{s}}^{H}\left(e^{-\Lambda}\right)$. Recall that for $g \in C(K)$ and $f \in C_{c}^{\infty}(H)$,

$$
\pi(f) \cdot g\left(k_{1}\right)=\int_{K} \int_{s} f\left(k_{1} p k_{2}\right) e^{\langle\Lambda \mid p\rangle} \cdot g\left(k_{2}^{-1}\right) d p d k_{2}
$$

That is $\pi(f)$ is given by the integral operator

$$
\begin{aligned}
\hat{F}(\Lambda)\left(k_{1}, k_{2}\right) & =\int_{\mathfrak{s}} f\left(k_{1} p k_{2}\right) e^{\langle\Lambda \mid p\rangle} d p \text { or } \\
\hat{F}(\Lambda) & =\int_{\mathfrak{s}} F(p) e^{\langle\Lambda \mid p\rangle} d p .
\end{aligned}
$$

Note that $\hat{F}(\operatorname{Ad} k \cdot \Lambda)=\tau_{1}(k) \hat{F}(\Lambda) \tau_{2}\left(k^{-1}\right)$ and $\hat{F}$ is (weakly) holomorphic in $\Lambda$.

Now let us do Fourier inversion on $\hat{F}(\Lambda)$. Let $q \in \mathfrak{s}$ and let $J(\Lambda)$ denote the appropriate Jacobian. Then

$$
\begin{aligned}
F(q) & =c \int_{\mathfrak{s}^{\vee}} \hat{F}(i \Lambda) e^{-\langle i \Lambda \mid q\rangle} d \Lambda \\
& =c \int_{K} \int_{\mathfrak{a}_{\mathfrak{g}}} \hat{F}(\operatorname{Ad} k \cdot i \Lambda) e^{-\langle i \operatorname{Ad} k \cdot \Lambda \mid q\rangle} J(\Lambda) d \Lambda d k \\
& =c \int_{\mathfrak{a}_{\mathfrak{g}}} \int_{K} \tau_{1}\left(k^{-1}\right) \hat{F}(i \Lambda) \tau_{2}(k) e^{-\langle i \Lambda \mid \operatorname{Ad} k \cdot q\rangle} d k J(\Lambda) d \Lambda .
\end{aligned}
$$


But now let $x=(l, q) \in H$ and let $\hat{F}(\Lambda)$ be a smooth integral operator on $\operatorname{ind}_{\mathbf{s}}^{H}\left(e^{-i \Lambda}\right)$ with $\Lambda$ real. Then $\pi(x)^{*}=\pi\left(x^{-1}\right)$ and

$$
\pi\left(x^{-1}\right) \hat{F}(\Lambda)\left(k_{1}, k_{2}\right)=\hat{F}(\Lambda)\left(l k_{1}, k_{2}\right) e^{-\left\langle i \Lambda \mid \operatorname{Ad} k_{1}^{-1} \cdot q\right\rangle}
$$

as an integral operator. Thus the trace is given by

$$
\operatorname{tr}\left(\pi(x)^{*} \hat{F}(i \Lambda)\right)=\int_{K} \tau_{1}\left(k^{-1}\right) \hat{F}(i \Lambda) \tau_{2}(k)(l, 1) e^{-\langle i \Lambda \mid \operatorname{Ad} k \cdot q\rangle} d k .
$$

Since the Plancherel measure and $J(\Lambda)$ agree up to a constant, we see that the operator valued Fourier transform and its inverse are given by

$$
\begin{array}{ll}
f \mapsto \hat{F}, & \hat{F}(\Lambda)=\int_{\mathfrak{s}} F(p) e^{\langle\Lambda \mid p\rangle} d p \\
\hat{F} \mapsto f, & f(l, q)=\int_{\mathfrak{s}^{\vee}} \hat{F}(i \Lambda) e^{-\langle i \Lambda \mid q\rangle} d \Lambda(l, 1)
\end{array}
$$

up to constant multiples.

Let $\mathfrak{D}$ be a finite subset of $\hat{K} \times \hat{K}$ and let $C_{c}^{\infty}(H, \mathfrak{D})$ be the space of $C_{c}^{\infty}$ functions on $H$ which transform according to $\mathfrak{D}$ under left and right translation (i.e., $f=$ $\left.\sum_{\left(\mathfrak{o}_{1}, \mathfrak{D}_{2}\right) \in \mathfrak{D}} \alpha_{\mathfrak{D}_{1}} * f * \alpha_{\mathfrak{D}_{2}}\right)$. Note that $f$ transforms according to $\mathfrak{D}$ if and only if $F$ does if and only if $\hat{F}$ does. Let $V, \tau$ be the unitary double $K$-submodule of $C^{\infty}(K \times K)$ consisting of those functions which transform according to $\mathfrak{D}$ under $\left(\tau_{1}, \tau_{2}\right)$, equipped with the $L_{2}$ norm. Then the ordinary Euclidean Paley-Wiener theorem gives: The operator valued Fourier transform of $C_{c}^{\infty}(H, \mathfrak{D})$ is the space of entire functions $\hat{F}: \mathfrak{s}_{c}^{\vee} \rightarrow V$ satisfying

$$
\hat{F}(\operatorname{Ad} k \cdot Z)=\tau_{1}(k) \hat{F}(Z) \tau_{2}\left(k^{-1}\right)
$$

and $\hat{F}$ is rapidly decreasing and of exponential type $r$ for some $r>0$. (That is, for each integer $N \geq 0$ there is a constant $C_{N}$ such that

$$
\left.\|\hat{F}(Z)\| \leq C_{N}\left(1+|Z|^{2}\right)^{-N} e^{r|\Re Z|} \quad\left(Z \in \mathfrak{s}_{c}^{\vee}\right) .\right)
$$

Let $G$ be the restriction of such an $F$ to $\mathfrak{a}_{\mathfrak{s} c}$. Then clearly $G$ is entire and rapidly decreasing and of exponential type $r$ (same $r$ and $C_{N}$ but now $Z \in \mathfrak{a}_{s c}^{\vee}$ ). Also if we write $\hat{F}=\sum_{m} \hat{F}_{m}$ as a Taylor series of homogeneous polynomials $\hat{F}_{m}$ of degree $m$ and set $G_{m}=\hat{F}_{m} \mid \mathfrak{a}_{\mathfrak{s} c}^{\vee}$, then $G=\sum_{m} G_{m}$ is the homogeneous Taylor series of $G$. Moreover each $\hat{F}_{m}$ lies in $S(\mathfrak{s}, \tau)$ so each $G_{m}$ is in $I\left(\mathfrak{a}_{\mathfrak{s}}, \tau\right)$ (see Theorem 19). Thus we have shown one direction in the following theorem.

THEOREM 35. Let $G: \mathfrak{a}_{\mathfrak{s c} c}^{\vee} \rightarrow V$ be an entire function. Then $G$ is the operator valued Fourier transform of a function $f \in C_{c}^{\infty}(H, \mathfrak{D})$ if and only if $G$ is rapidly decreasing and of exponential type $r$ (for some $r>0$ ) and each $G_{m}$ lies in $I\left(\mathfrak{a}_{\mathfrak{s}}, \tau\right)$, where $G=\sum_{m} G_{m}$ is the Taylor series of $G$ with each $G_{m}$ homogeneous of degree $m$.

PROOF. A similar result, at least in the zonal case, appears in [H2, pp. 299, 313]. Using results from there, we prove it by showing that $G$ extends to an entire function $\hat{F}$ on $\mathfrak{s}_{c}$ satisfying the conditions listed before the lemma.

(1) First we show that an entire function $G=\sum_{m} G_{m}: \mathfrak{a}_{s c}^{\vee} \rightarrow V$ with each $G_{m}$ in $I\left(\mathfrak{a}_{\mathfrak{s}}, \tau\right)$ extends to an entire function on $\mathfrak{s}_{c}$. An argument similar to the proof of 
Lemma 11 shows that $G$ extends to a holomorphic function on the set $\mathfrak{s}_{c}^{\prime}$ of $s$-regular elements. If $H$ corresponds to a rank one group, then Lemma 16, and especially step (10) in its proof, shows that $I\left(\mathfrak{a}_{\mathfrak{s}}, \tau\right)$ is a free module over the algebra $I\left(\mathfrak{a}_{\mathfrak{s}}\right)$ of Weyl group invariants. Thus if $v_{i}$ is a basis for $V_{M}$ with $P\left(\mathfrak{d}_{i \lambda}\right) P\left(\mathfrak{d}_{i 2 \lambda}\right) \cdot v_{i}=v_{i}$ and if $k_{i}=k\left(\mathfrak{d}_{i \lambda}, \mathfrak{d}_{i 2 \lambda}\right)$ as in Lemma 16 , then

$$
G(\lambda)=\sum v_{i} \otimes \lambda^{k_{i}} G_{i}(\lambda)
$$

where each $G_{i}(\lambda)$ is entire and Weyl group invariant. Each $v_{i} \otimes \lambda^{k_{i}}$ extends to a polynomial function $p_{i} \in S\left(\mathfrak{s}_{c}, \tau\right)$ and by [H2, p. 299] each $G_{i}$ extends to a $K_{c^{-}}$ invariant entire function $F_{i}$ on $\mathfrak{s}_{c}$. Then $F=\sum p_{i} F_{i}$ provides an entire extension of $G$ to $\mathfrak{s}_{c}^{\vee}$ satisfying $F(\operatorname{Ad} k \cdot Z)=\tau_{1}(k) F(Z) \tau_{2}\left(k^{-1}\right)$ (rank one case). Now steps (4) and (5) in the proof of Theorem 19 go through to show that $G$ extends to $\mathfrak{s}_{c}^{\vee}$ in any rank to an entire function satisfying the $K_{c}$-equivariance condition.

(2) Next we show that the extension $F$ is of exponential type $r$. Let $(\cdot \mid \cdot)$ be the Hermitian inner product on $V$ (conjugate linear in the second slot) making $\tau_{1}$ and $\tau_{2}$ unitary. Extend to a pairing

$$
(\cdot \mid \cdot): S\left(\mathfrak{s}_{c}, \tau\right) \times S\left(\mathfrak{s}_{c}, \tau\right) \rightarrow I\left(\mathfrak{s}_{c}\right) \quad \text { where }(f \mid g)(Z)=(f(Z) \mid g(\bar{Z})) .
$$

(The $K_{c}$-invariance comes from the fact that Ad $k$ commutes with complex conjugation $Z \mapsto \bar{Z}$ for $k \in K$, and $(f \mid g)$ is holomorphic.)

Let $v_{1}, \ldots, v_{n}$ be an orthonormal basis for $V_{M}$ and let $p$ be a polynomial function on $\mathfrak{a}_{\mathfrak{s} c}^{\vee}$ such that $p(\rho)=1$ and $p(w \cdot \rho)=0$ for $w \neq 1$ in $W_{\mathfrak{s}}$. Define the polynomials $g_{i}: \mathfrak{a}_{\mathfrak{s} c}^{\vee} \rightarrow V_{M}$ by

$$
g_{i}(\Lambda)=\sum \mu(w) \cdot v_{i} p\left(w^{-1} \cdot \Lambda\right)
$$

Then $g_{i}(w \cdot \Lambda)=\tau_{1}(w) g_{i}(\Lambda) \tau_{2}\left(w^{-1}\right)$ and, replacing $p$ by $p \pi^{2 n}$ for a sufficiently large $n$, Lemma 11 implies that we may assume that each $g_{i} \in I\left(\mathfrak{a}_{\mathfrak{s}}, \tau\right)$. (If we assume $n \geq 1$ then condition (1), there, is automatically satisfied since then $g_{i}$ vanishes identically on the singular set.) Let $\tilde{g}_{i}: \mathfrak{s}_{c} \rightarrow V$ be the $K_{c}$-equivariant extensions and define the matrix $M$ of $K_{c}$-invariant polynomials by $M_{i j}=\left(g_{i} \mid g_{j}\right)$. Let $m=\operatorname{det} M$ and let $M^{\dagger}$ be the adjoint matrix, so $M \cdot M^{\dagger}=m \cdot 1$. Define the function

$$
\Phi=\sum_{i j}\left(F \mid \tilde{g}_{i}\right) M_{i j}^{\dagger} \tilde{g}_{j}: \mathfrak{s}_{c} \rightarrow V
$$

so $\Phi$ is entire and $\Phi(\operatorname{Ad} k \cdot Z)=\tau_{1}(k) \Phi(Z) \tau_{2}\left(k^{-1}\right)$.

By construction, $\left(g_{i} \mid g_{j}\right)(\rho)=\left(v_{i} \mid v_{j}\right)=\delta_{i j}$, so there is a Zariski open set $U$, the nonzeros of $m$ in $\mathfrak{a}_{\mathfrak{s c}}^{\vee}$, such that the $g_{i}(h)$ form a basis for $V_{M}$ when $h \in U$. Thus for $v \in V_{M}$ and $h \in U$ we have

$$
m(h) \cdot v=\sum_{i j}\left(v \mid g_{i}(h)\right) M_{i j}^{\dagger}(h) g_{j}(h) .
$$

This implies that $\Phi\left|\mathfrak{a}_{s c}^{\vee}=m\right| \mathfrak{a}_{s c}^{\vee} \cdot F \mid \mathfrak{a}_{s c}^{\vee}$ and, since restriction is clearly one to one, that $\Phi=m F$.

Suppose $Z \in \mathfrak{s}_{c}^{\vee}$ is $s$-regular. The function $k \mapsto|\mathfrak{R}(\operatorname{Ad} k \cdot Z)|$ (for $k \in K_{c}$ ) is minimized when $\operatorname{Ad} k \cdot Z \in \mathfrak{a}_{\mathfrak{s} c}^{\vee}$, as is shown in [H2, p. 300] (using $\mathfrak{R}(Z)=\mathfrak{I}(i Z)$ ). 
The functions $\sum_{i}\left(F \mid \tilde{g}_{i}\right) M_{i j}^{\dagger}$ are holomorphic and $K$-invariant, hence $K_{c}$-invariant, and for $Z=\operatorname{Ad} k \cdot \Lambda$

$$
\begin{aligned}
& \left|\sum_{i}\left(F \mid \tilde{g}_{i}\right) M_{i j}^{\dagger}(Z)\right|=\left|\sum_{i}\left(G(\Lambda) \mid g_{i}(\Lambda)\right) M_{i j}^{\dagger}(\Lambda)\right| \\
& \quad \leq \sum_{i}\left|g_{i}(\Lambda)\right|\left|M_{i j}^{\dagger}(\Lambda)\right| C_{N}\left(1+|\Lambda|^{2}\right)^{-N} e^{r|\mathfrak{R} \Lambda|} \\
& \quad \leq C_{j} e^{r|\Re \Lambda|}
\end{aligned}
$$

(choosing $N$ large enough so that $\sum\left|g_{i}\right|\left|M_{i j}^{\dagger}\right|\left(1+|\cdot|^{2}\right)^{-N}$ remains bounded). So now

$$
\|m(Z) F(Z)\| \leq \sum_{j}\left|g_{j}(Z)\right| C_{j} e^{r|\Re \Lambda|}
$$

for any $Z$ which is $s$-regular. By continuity, this holds for any $Z \in \mathfrak{s}_{c}^{\vee}$ and then [T, p. 274] implies that $F$ itself is slowly growing and of exponential type $r$.

(3) The last sentence implies, by the Euclidean Paley-Wiener theorem, that $F$ is the Fourier transform of a tempered distribution with compact support in the ball of radius $r$. On the other hand if $Z=i$ Ad $k \cdot \Lambda$ lies in the unitary axis $i \mathfrak{s}$ (where $\Lambda \in \mathfrak{a}_{\mathbf{s}}^{\vee}$ ) then

$$
\|F(Z)\|=\|G(i \Lambda)\| \leq C_{N}\left(1+|\Lambda|^{2}\right)^{-N}
$$

for all $N$, so $F$ is also the Fourier transform of a $C^{\infty}$ function all of whose derivatives are $L_{2}$. This implies the theorem.

Now drop the assumption that $f$ is $K$-finite, and let $f \in C_{c}^{\infty}(H)$ be supported in $K \times B_{r}$, where $B_{r}$ is the ball of radius $r$ in $\mathbf{s}$. Then again we have the operator valued Fourier transform $G: \mathfrak{a}_{s c}^{\vee} \rightarrow L_{2}(K \times K)$ given by (34) with $\Lambda \in \mathfrak{a}_{\mathfrak{s c}}^{\vee}$. The easy direction of the proof of the ordinary Paley-Weiner theorem and [W1, p. 261] imply that $G$ is (weakly) entire and for any $N \in \mathbf{N}$ there is a constant $C_{N}>0$ such that

$$
\left\|P\left(\mathfrak{d}_{1}\right) G(\Lambda) P\left(\mathfrak{d}_{2}\right)\right\|_{2} \leq C_{N}\left(1+\left|\mathfrak{d}_{1}\right|^{2}\right)^{-N}\left(1+\left|\mathfrak{d}_{2}\right|^{2}\right)^{-N}\left(1+|\Lambda|^{2}\right)^{-N} e^{r|\Re \Lambda|}
$$

where $|\mathfrak{d}|^{2}$ is defined by $\mu_{\mathfrak{o}}\left(\omega_{k}\right) \cdot P(\mathfrak{d})=|\mathfrak{d}|^{2} P(\mathfrak{d})$ and $\omega_{k}$ is the Casimir operator on $\mathfrak{k}$ computed relative to the (negative definite) restriction of the Killing form $B$. Moreover Theorem 35 tells us that the homogeneous Taylor components of $P\left(\mathfrak{d}_{1}\right) G(\cdot) P\left(\mathfrak{d}_{2}\right)$ lie in $I\left(\mathfrak{a}_{\mathfrak{s}}, \tau\right)$ for each $\mathfrak{d}_{1}, \mathfrak{d}_{2}$ (where $V, \tau$ is the subspace of $L_{2}(K \times K)$ which transforms according to $\mathfrak{d}_{1}$ under $\tau_{1}$ and $\mathfrak{d}_{2}$ under $\tau_{2}$ ).

Conversely, if $G: \mathfrak{a}_{\mathfrak{s c}}^{\vee} \rightarrow L_{2}(K \times K)$ has the above properties, then Theorem 35 tells us that each $P\left(\mathfrak{d}_{1}\right) G(\cdot) P\left(\mathfrak{d}_{2}\right)$ is the operator valued Fourier transform of a function $f_{\mathfrak{o}_{1}, \mathfrak{o}_{2}}$ in $C_{c}^{\infty}(H)$ supported in $K \times B_{r}$ and satisfying $f_{\mathfrak{o}_{1}, \mathfrak{o}_{2}}=\alpha_{\mathfrak{o}_{1}}$ * $f_{\mathfrak{o}_{1}, \mathfrak{o}_{2}} * \alpha_{\mathfrak{o}_{2}}$. Now the Sobolev norms generate the $C_{c}^{\infty}(H)$ topology on the space of functions supported in the fixed compact set $K \times B_{r}\left[\mathbf{T}\right.$, p. 64]: $\cap H_{\text {loc }}^{s}=C^{\infty}$ topologically). Thus the Fourier series

$$
f=\sum_{\left(\mathfrak{o}_{1}, \mathfrak{o}_{2}\right) \in \hat{K} \times \hat{K}} f_{\mathfrak{o}_{1}, \mathfrak{o}_{2}}
$$

is absolutely convergent in $C_{c}^{\infty}(H)$ to a smooth function $f$ with support in $K \times B_{r}$. 
Summarizing, we have shown

COROLlaRY 37. Let $G: \mathfrak{a}_{s c}^{\vee} \rightarrow L_{2}(K \times K)$ be a (weakly) entire function. Then $G$ is the operator valued Fourier transform of a function $f \in C_{c}^{\infty}(H)$ if and only if for each $N \in \mathbf{N}, G$ satisfies an inequality of the form (36), and for each $\mathfrak{d}_{1}, \mathfrak{d}_{2} \in \hat{K}$ the homogeneous Taylor components of $P\left(\mathfrak{d}_{1}\right) G(\cdot) P\left(\mathfrak{d}_{2}\right)$ lie in $I\left(\mathfrak{a}_{\mathfrak{s}}, \tau\right)$ (where $V=$ $\left.P\left(\mathfrak{d}_{1}\right) L_{2}(K \times K) P\left(\mathfrak{d}_{2}\right)\right)$.

APPENDIX. Some algebraic lemmas. Here we intend to state some general algebraic lemmas which were used in the body of the paper. For the proofs, the reader is referred to $[\mathbf{R}]$.

Lemma. Let $X \subseteq A$ be algebras with 1 over $\mathbf{C}$ and let $Z$ be a Noetherian subalgebra of $X$ which is contained in the center of $A$ with $1 \in Z$. Assume that the vector space $A / X$ is finite dimensional. Let $\eta: Z \rightarrow \mathbf{C}$ be a representation and let $M$ be the kernel of $\eta$. If $\alpha: X \rightarrow A$ is the inclusion, let $\alpha^{\prime}: X / M X \rightarrow A / M A$ be the natural map induced by $\alpha$. Then the kernel of $\alpha^{\prime}$ is contained in the (nilpotent) radical of $X / M X$.

The proof of this lemma is by localizing at $M$. The finite dimensionality of $A / X$ is used only to guarantee that the $Z$-module $A / X$ has a locally finite composition series in which each factor is a semisimple $Z$-module.

LEMMA. Let $B$ be a finite dimensional algebra over $\mathbf{C}, 1 \in B$, and let $\phi_{n}: B \rightarrow$ $\mathscr{L}\left(E_{n}\right)$ be finite dimensional representations of $B$ such that $\bigcap$ ker $\phi_{n}$ is a nilpotent ideal in $B$. Then every irreducible representation of $B$ occurs in the Jordan-Hölder series of one of the $\phi_{n}$.

The proof uses the Chinese remainder theorem.

LEMMA. Let the notation and assumptions be as in the previous two lemmas, with $B=X / M X$. Assume in addition that $A$ is a finite module over $Z$. Let $\phi$ be an irreducible representation of $X$ such that $\phi \mid Z=\eta \cdot 1$. Let $\phi_{n}$ be the irreducible representations of $A$ such that $\phi_{n} \mid Z=\eta \cdot 1$. Then $\phi$ occurs in the Jordan-Hölder series of one of the $\phi_{n} \mid X$.

For the proof, note that $\phi_{n}$ and $\phi$ factor through the finite dimensional algebras $A / M A$ and $B=X / M X$, and $A / X$ is finite dimensional. The $\phi_{n}$ are all of the irreducible representations of $A / M A$, so $\bigcap \operatorname{ker} \phi_{n}$ is a nilpotent ideal. But then $\cap \alpha^{\prime-1}\left(\operatorname{ker} \phi_{n}\right)$ is a nilpotent ideal in $X / M X$ by the first lemma, and so the result follows from the second.

Lemma. Let $A$ be a finite dimensional algebra over $\mathbf{C}$ and let $W$ be a finite group of automorphisms of $A$. Let $B$ be the subalgebra of $W$-invariants in $A$. Let $\phi: A \rightarrow \mathscr{L}(F)$ be an irreducible representation of $A$. Then $\phi \mid B$ is a semisimple representation of $B$. In particular if $A$ is semisimple then so is $B$.

Proof. For $w \in W$ define ${ }^{w} \phi: A \rightarrow \mathscr{L}(F)$ by ${ }^{w} \phi(a)=\phi\left(w^{-1} \cdot a\right)$, an irreducible representation of $A$. Let $W_{\phi}$ be the subgroup of $w \in W$ such that ${ }^{w} \phi$ is equivalent to $\phi$, and for each $w \in W_{\phi}$ let $S_{w}$ be an equivalence, $\phi(a) S_{w}=S_{w}{ }^{w} \phi(a)$. Then $w \mapsto S_{w}$ defines a projective representation of $W_{\phi}$ on $F$.

Choose $w_{1}, \ldots, w_{r} \in W$, representatives of $W / W_{\phi}$ and choose $e_{i} \in A$ such that ${ }^{w_{i}} \phi\left(e_{j}\right)=\delta_{i j} \cdot 1$. Let $\mathscr{S}=\sum\left\{\mathbf{C} \cdot S_{w} \mid w \in W_{\phi}\right\}$ and $L \in \mathscr{L}(\boldsymbol{F})$ and suppose 
$L \circ S=S \circ L$ for all $S \in \mathscr{S}$. Choose $a_{i} \in A$ such that ${ }^{w_{i}} \phi\left(a_{i}\right)=L$ and put $a=\sum e_{i} a_{i}$, so ${ }^{w_{i}} \phi(a)=L$ for all $i$. Since $L \circ S=S \circ L$ for all $S \in \mathscr{S}$ we have $S_{w} \circ{ }^{w_{i}} \phi(a)={ }^{w_{i}} \phi(a) \circ S_{w}=S_{w} \circ{ }^{w_{i} w} \phi(a)$ for all $w \in W_{\phi}$, so

$$
w_{i} \phi(a)=w_{i} w \phi(a) \text {. }
$$

Thus

$$
\begin{aligned}
L & =\left[W: W_{\phi}\right]^{-1} \sum_{i}{ }^{w_{i}} \phi(a) \\
& =\left[W: W_{\phi}\right]^{-1}\left[W_{\phi}\right]^{-1} \sum_{i} \sum_{w \in W_{\phi}} w_{i} w \phi(a)=\phi(b)
\end{aligned}
$$

where

$$
b=[W]^{-1} \sum_{w \in W} w \cdot a \in B .
$$

Thus $\phi(B)$ is the commuting algebra of $\mathscr{S}$, say $\mathscr{S}^{\prime}$. But now $\mathscr{S}$ is the image of the group algebra of $W_{\phi}$ under a projective representation, and since $\mathbf{C}$ is algebraically closed and of characteristic zero, we have $\mathscr{S}=\mathscr{S}^{\prime \prime}=\phi(B)^{\prime}$ which is semisimple. This implies the result.

Note that by breaking the irreducible representations of $A$ up into orbits under $W$, one could determine the irreducible representatione of $B$ in terms of those of $A$.

Finally we have the following analogue of the Jacobson density theorem.

Proposition. Let $A$ be an associative algebra over $\mathbf{C}$ and let $\pi: A \rightarrow \mathscr{L}(E)$ be a representation, where $E$ is a Fréchet space (so the open mapping theorem holds). Suppose $\pi$ is topologically irreducible and that $1 \in \mathscr{L}(E)$ lies in the closure of $\pi(A)$ in the topology of simple convergence. Suppose that the only continuous operators which intertwine $\pi$ are scalar multiples of the identity. Then $\pi$ is TCI.

PROOF. Let $E^{n} \simeq \mathbf{C}^{n} \otimes E$ be the direct sum of $n$ copies of $E$ with the product topology. Let $\pi^{n}=1 \otimes \pi: A \rightarrow \mathscr{L}\left(E^{n}\right)$ be the diagonal representation of $A$ deduced form $\pi$.

(1) Let $F$ be a closed $\pi^{n}$-invariant subspace of $E^{n}$. Then I claim that there is another closed submodule $F^{\prime} \subseteq E^{n}$ such that $E^{n}=F \oplus F^{\prime}$ (topologically). To see this, write $E^{n}=E_{1} \oplus \cdots \oplus E_{n}$ where each $E_{i} \simeq E$. Let $I=\{1, \ldots, n\}$ and let $J$ be a maximal subset of $I$ such that $F+\sum_{j \in J} E_{j}$ is a direct sum. For each $i \in I$, $E_{i} \cap\left(F+\sum_{j \in J} E_{j}\right)$ is a closed submodule of $E_{i}$, hence equal to $E_{i}$ or zero. But if it were zero we would get a contradiction to the maximality of $J$. Put $F^{\prime}=\sum_{j \in J} E_{j}$ with the product topology. Then $E^{n}=F \oplus F^{\prime}$ algebraically. Also with the product topology on $F \oplus F^{\prime}$, the obvious map of $F \oplus F^{\prime}$ onto $E^{n}$ is continuous, one-to-one and onto. Thus by the open mapping theorem $E^{n} \simeq F \oplus F^{\prime}$ is an isomorphism of topological vector spaces and of $A$-modules.

(2) Let $M=\mathscr{L}\left(\mathbf{C}^{n}\right) \otimes 1 \subseteq \mathscr{L}\left(E^{n}\right)$. If $Q \in \mathscr{L}\left(E^{n}\right)$ commutes with $\pi^{n}$ then $Q \in M$. Also $S \in \mathscr{L}\left(E^{n}\right)$ commutes with $M$ if and only if there is a $T \in \mathscr{L}(E)$ such that $S=1 \otimes T$. This is easy to verify from the hypothesis of Schur's lemma for bounded operators.

(3) Suppose $S \in \mathscr{L}\left(E^{n}\right)$ commutes with $M$ and let $e \in E^{n}$. I claim that there is a net $a_{p} \in A$ such that $\pi^{n}\left(a_{p}\right) \cdot e$ converges to $S \cdot e$. To see this, let $F$ be the closure of the subspace $\left\{\pi^{n}(a) \cdot e \mid a \in A\right\}$. Then $F$ is $\pi^{n}$-invariant so we can choose 
a $\pi^{n}$-invariant closed subspace $F^{\prime}$ with $E^{n} \simeq F \oplus F^{\prime}$. Let pr be the projection of $E^{n}$ onto $F$ with kernel $F^{\prime}$. Since the direct sum is topological, pr is continuous and commutes with $\pi^{n}$. Thus pr $\in M$ and $\mathrm{pr} \cdot e=e$ (since 1 is in the simple closure of $\pi^{n}(A)$ so $\left.e \in F\right)$. Thus

$$
S \cdot e=S \circ \mathrm{pr} \cdot e=\operatorname{pr} \circ S \cdot e \in F
$$

so there is a net $a_{p} \in A$ such that $\pi^{n}\left(a_{p}\right) \cdot e \rightarrow S \cdot e$.

(4) Combining (2) and (3) we have shown that if $e_{1}, \ldots, e_{n} \in E$ and $T \in \mathscr{L}(E)$ then there is a net $a_{p} \in A$ such that $\pi\left(a_{p}\right) \cdot e_{i}$ converges to $T \cdot e_{i}$. This is one version of the definition of TCI [W1, p. 228].

\section{REFERENCES}

[BC] D. Bartlet and J. L. Clerc, Le comportement a l'infini des fonctions de Bessel generalisees. I, preprint, Institut Elie Cartan, Universite de Nancy, 1981, pp. 1-25.

[C] J. L. Clerc, Le comportement a l'infini des fonctions de Bessel generalisees. II, preprint, Institut Elie Cartan, Universite de Nancy, 1981, pp. 1-40.

[D] J. Dixmier, Les $C^{*}$-algèbres et leurs représentations, Gauther-Villars, Paris, 1964.

[DKV] J. A. C. Kolk, J. J. Duistermaat and V. S. Varadarajan, Functions, flows and oscillatory integrals on flag manifolds and conjugacy classes in real semisimple Lie groups, Compositio Math. 49 (1983), 309-398.

[F1] J. M. G. Fell, The dual spaces of Banach algebras, Trans. Amer. Math. Soc. 114 (1965), 227-250.

[F2] _ Non-unitary dual spaces of groups, Acta Math. 114 (1965), 267-310.

[G] R. Godement, $A$ theory of spherical functions. I, Trans. Amer. Math. Soc. 73 (1952), 496-556.

[G\&R] R. C. Gunning and H. Rossi, Analytic functions of several complex variables, Prentice-Hall, Englewood Cliffs, N.J., 1965.

[Gr] A. Grothendieck, Sur quelques points d'algèbre homologique, Tôhoku Math. J. 9 (1957), 119221.

[H] S. Helgason, Differential geometry and symmetric spaces, Academic Press, New York, 1962.

[H2] __, A duality for symmetric spaces with applications to group representations. III, Tangent space analysis, Advances in Math. 36 (1980), 297-323.

[HCI] Harish-Chandra, Harmonic analysis on real reductive groups (I), J. Funct. Anal. 19 (1975), 104-204.

[HCIII] - Harmonic analysis on real reductive groups (III), Ann. of Math. (2) 104 (1976), 117-201.

[K\&L] A. Kleppner and R. L. Lipsman, The Plancherel formula for group extensions, Ann. Sci. École Norm. Sup. 5 (1972), 71-120, and 6 (1973), 103-132.

[K\&R] B. Kostant and S. Rallis, Orbits and representations associated with symmetric spaces, Amer. J. Math. 93 (1971), 753-809.

[L] J. Lepowski, Algebraic results on representations of semi-simple Lie groups, Trans. Amer. Math. Soc. 176 (1973), 1-44.

[M] A. Melin and J. Sjöstrand, Fourier integral operators with complex valued phase functions, Lecture Notes in Math., vol. 459, Springer-Verlag, Berlin and New York, 1975, pp. 120-223.

[R] C. Rader, Spherical functions on a semi-simple Lie group, Univ. of Chicago Lecture Notes, Chicago, Ill., 1976.

[S] S. Sakai, On the representations of semi-simple Lie groups, Proc. Japan Acad. 30 (1954), 14-19.

[T] F. Trèves, Linear partial differential equations with constant coefficients, Gordon and Breach, New York, 1966.

[W] N. Wallach, Harmonic analysis on homogeneous spaces, Marcel Dekker, Princeton, N.J., 1973.

[Weyl] H. Weyl, The classical groups, Princeton Univ. Press, Princeton, N.J., 1946.

[W1,W2] G. Warner, Harmonic analysis on semi-simple Lie groups, vols. I and II, Springer-Verlag, Berlin and New York, 1972. 